\title{
Time-Interleaved High Speed D/A Converters
}

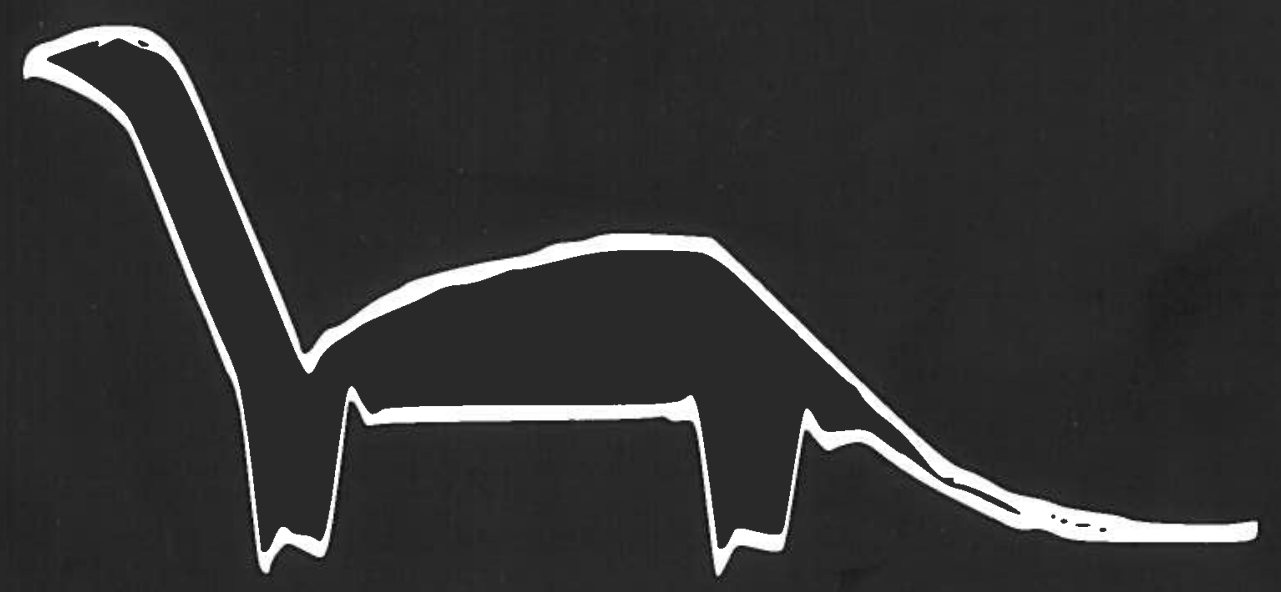

\section{Erik Olieman}




\section{Time-Interleaved High-speed D/A Converters}

Erik Olieman 
Samenstelling promotiecommissie:

Voorzitter en secretaris:

Prof.dr. P.M.G. Apers

Promotor:

Prof.dr.ir B. Nauta

Universiteit Twente

Universiteit Twente

Assistent-promotor:

Dr.ir. A.J. Annema

Universiteit Twente

Leden:

Prof.dr. K.A.A. Makinwa

Technische Universiteit Delft

Prof.dr.ir. F.E. van Vliet

Universiteit Twente

Prof.ir. A.J.M. van Tuijl

Prof.dr.ir. K. Doris

Universiteit Twente

Technische Universiteit Eindhoven

This research is conducted as part of the Sensor STARS $\%$. Technology Applied in Reconfigurable systems for sustainable Security (STARS) project, see also www.starsproject.nl.

Centre for Telematics and Information Technology

P.O. Box 217, $7500 \mathrm{AE}$

Enschede, The Netherlands

ISBN: 978-90-365-4019-3

DOI: http://dx.doi.org/10.3990/1.9789036540193

Copyright (C) 2015 by Erik Olieman, Enschede, The Netherlands 


\title{
TIME-INTERLEAVED HIGH- SPEED D/A CONVERTERS
}

\section{PROEFSCHRIFT}

\author{
Ter verkrijging van \\ de graad van doctor aan de Universiteit Twente, \\ op gezag van de rector magnificus, \\ prof. dr. H. Brinksma, \\ volgens besluit van het College voor Promoties \\ in het openbaar te verdedigen \\ op woensdag 2 maart 2016 om 14:45
}

door

Erik Olieman

geboren op 28 mei 1989

te Wageningen 
Dit proefschrift is goedgekeurd door:

De promotor:

De assistent-promotor:
Prof. dr. Ir. Bram Nauta

Dr.ir. A.J. Annema 


\section{Samenvatting}

Het onderwerp van deze scriptie zijn energie efficiënte, snelle digitaal-naar-analoog converters (DACS) in CMOS technologie, die de mogelijkheid bieden om signalen van $\mathrm{DC}$ tot RF te genereren. Tegenwoordig worden componenten in RF ketens bij voorkeur in het digitale domein geplaatst in plaats van in het analoge domein. Dit levert voordelen op qua flexibiliteit en betere prestaties bij nieuwere CMOS technologieën. Welke taken naar het digitale domein kunnen worden verplaatst hangt, voor een significant gedeelte, af van de prestaties van de aanwezige DACs.

Het werk aan deze scriptie was onderdeel van het STARS project, wat als doel heeft om technologieën voor re-configureerbare radar systemen te ontwikkelen. De mogelijkheid om meer taken in het digitale domein uit te voeren, wat kan wanneer betere DACs ontwikkeld worden, past goed bij de doelen van het STARS project.

De meest gebruikte DAC techniek voor generatie van hoog frequente signalen is de stroom-sturende DAC. De werking en de beperkingen van dit type DAC wordt besproken in deze thesis, inclusief een aantal bekende oplossingen uit de literatuur voor de beperkingen van deze DACs. Er wordt aangetoond dat de primaire foutmechanismes plaatsvinden op, of direct na, het moment waarbij er naar nieuwe data wordt geschakeld. Voor de rest van de periode van de DAC, zodra de uitgang zijn definitieve waarde heeft aangenomen, is de uitgang nagenoeg ideaal. Deze fouten gerelateerd aan het schakelgedrag van DAC beperken de te behalen bandbreedte en energie efficiëntie van conventionele DACs.

Interleaved stroom-sturende DACs, het onderwerp van deze thesis, worden geïntroduceerd om de beperkingen in nauwkeurigheid door schakel fouten te onderdrukken. Interleaved DACs bestaan uit twee sub-DACs die parallel hun werk doen, en door een analoge multiplexer gecombineerd worden tot één uitgang. De sDACs zijn standaard stroom-sturende DACs, die door hun niet-ideale gedrag een beperkte nauwkeurigheid hebben. Nadat één van de sDACs een nieuwe digitale code 
krijgt om te converteren, krijgt hij in de interleaved DAC wat tijd om naar zijn definitieve waarde te convergeren. Gedurende deze periode is de andere sDAC, die op de tegenovergestelde fase van de klok werkt, verbonden met de uitgang via de analoge multiplexer. Zodra de eerste SDAC zijn definitieve waarde heeft bereikt, schakelt de analoge multiplexer en wordt deze eerste SDAC met de uitgang verbonden. Ondertussen krijgt de tweede SDAC een nieuwe digitale code, en krijgt die tijd om naar zijn uiteindelijke waarde te convergeren. Deze architectuur isoleert de dominante fouten van de sDACs, die rond het schakelmoment optreden, van de daadwerkelijke uitgang.

De interleaved architectuur resulteert in een hogere lineariteit van hoge-snelheids DACs, echter er zijn ook een aantal beperkingen waar rekening mee gehouden moet worden. De analoge multiplexer moet voldoende lineair zijn, de amplitude van de twee sDACs moet soortgelijk zijn, en ook de duty cycle van de twee sDACs moet nauwkeurig gedefinieerd zijn. Zoals beschreven in hoofdstuk 3, het gedrag van de analoge multiplexer is code-onafhankelijk door gebruik te maken van triode schakelaars in plaats van saturatie schakelaars. Daarnaast wordt er ook een methode geïntroduceerd in dit hoofdstuk die de fout in de duty cycle kan bepalen en met alleen DC vergelijkingen.

Om de effectiviteit van de interleaved architectuur aan te tonen is er een 1.7GS/s, 12bit DAC ontworpen die een SFDR van meer dan $58 \mathrm{~dB}$ heeft over zijn complete Nyquist bandbreedte, en hierbij $70 \mathrm{~mW}$ nodig heeft. De individuele stroombronnen van de sDACs kunnen worden gekalibreerd met een nieuwe methode waarbij enkel DC vergelijkingen nodig zijn. De initiële ongekalibreerde fout in de uitgangsamplitudes van de sDACs is 60LSB, terwijl na kalibratie dit met een factor 150 is gereduceerd tot slechts 0.4 LSB. Een digitaal gestuurde condensator bank is aanwezig op de chip om de duty cycle aan te kunnen passen. Derde orde intermodulatie producten zijn met bijna $20 \mathrm{~dB}$ onderdrukt in de interleaved uitgang ten opzichte van de uitgang van de sDACs.

Hiernaast zijn nog twee ICs ontworpen. Beide hebben 9-bit resolutie, en meer dan 50dB SFDR over hun Nyquist bandbreedte terwijl ze $100 \mathrm{~mW}$ verbruiken. De eerste is ontworpen en geproduceerd in $65 \mathrm{~nm}$ CMOS, en werkt op een snelheid van $8.8 \mathrm{GS} / \mathrm{s}$, terwijl de tweede werkt op $11 \mathrm{GS} / \mathrm{s}$ en in $28 \mathrm{~nm}$ FDSOI is gemaakt. Beide DACs zijn erg klein, ze nemen respectievelijk $0.074 \mathrm{~mm}^{2}$ en $0.04 \mathrm{~mm}^{2}$ in. Deze efficiëntie is bereikt door gebruik te maken van quad-switching, waarmee ongewenste code-afhankelijke 
effecten verder onderdrukt worden. De duty cycle van deze DACs kan aangepast worden door een externe spanning aan te bieden.

Deze drie chips tonen aan dat, ondanks dat er twee sDACs nodig zijn, de interleaved architectuur erg geschikt is voor het ontwerpen van kleine, zuinige, DACs met goede performance bij hele hoge snelheden. 



\section{Abstract}

This thesis is on power efficient very high-speed digital-to-analog converters (DACs) in CMOS technology, intended to generate signals from DC to RF. Components in RF signal chains are nowadays often moved from the analog domain to the digital domain. This allows for more flexibility and better scaling of performance with new CMOS processes. The number of tasks in the transmit chain that can be moved to the digital domain depends on the performance of the available DACs.

This thesis was performed as part of the STARS project, which is intended to develop the technologies required for reconfigurable radar systems. The flexibility and reconfigurability which is possible in the digital domain fits well within the scope of the project, which can be better exploited with improved DACs.

The most widely used DAC implementation for high-speed operation is the current steering DAC. Its operating principles and its limitations are discussed in the introduction of this thesis, including some solutions reported in literature for these limitations. It is shown that the major error sources of conventional high-speed current steering DACs occur right at or after the switching instance, while for the majority of the DAC's period, after its output is settled, the output is close to ideal. These switching related errors limit the signal frequency range and power efficiency of conventional DACs considerably.

Interleaved current steering DACs, which are the topic of this thesis, are introduced to circumvent these performance-limiting switching related issues. The interleaved architecture consists of two sub-DACs that operate in parallel and an analog multiplexer that combines them into one output. The sDACs are regular current steering DACs, with limited performance due to their non-ideal behavior. After one of the sDACs is supplied with a new digital code, it gets some time to settle. During this time the other sDAC which operates at the opposite clock phase is connected to the output via the analog multiplexer. After the first SDAC is finished settling, the analog multiplexer toggles and 
connects that sDAC to the output. Meanwhile the second SDAC is supplied with a new digital code and gets time to settle its output. This architecture isolates the most dominant errors of the sDACs, which are centered around the switching instance, from the actual output.

This interleaved architecture allows for better linearity for high-speed DACs, but it also has some inherent limitations that need to be taken into account. The analog multiplexer should be sufficiently linear, the full scale amplitude of the sDACs need to be similar, and also the duty cycle of the two sDACs need to be accurately defined. As described in chapter 3 , code independent behavior of the analog multiplexer is achieved by employing triode switches instead of saturation switches. Also a method to measure the duty cycle error is introduced which only requires DC comparisons.

In order to proof the viability of the interleaved architecture a 1.7GS/s, 12-bit DAC is designed that achieves better than 58dB SFDR over Nyquist while consuming $70 \mathrm{~mW}$. The individual current sources of the SDACs can be calibrated with a new method using only DC comparisons. The initial uncalibrated gain error between the two SDACs is over 60LSB, while after calibration this is reduced by a factor 150 to only 0.4 LSB. A digital capacitor bank is included which is capable of adjusting the duty cycle to remove any duty cycle associated errors. The third order intermodulation products are reduced by almost $20 \mathrm{~dB}$ when the interleaved output is compared to the direct output of the sDACs.

Two more ICs are designed, both having 9-bit resolution, better than 50dB SFDR over Nyquist and close to $100 \mathrm{~mW}$ power consumption. The first one is produced in $65 \mathrm{~nm}$ CMOS and runs at $8.8 \mathrm{GS} / \mathrm{s}$, while the second one runs at $11 \mathrm{GS} / \mathrm{s}$ and is produced in $28 \mathrm{~nm}$ FDSOI. Their core area is very small: they occupy respectively $0.074 \mathrm{~mm}^{2}$ and $0.04 \mathrm{~mm}^{2}$. This power efficient high-speed operation is achieved by using quadswitching to further suppress unwanted code-dependent behavior. Their duty cycle can be adjusted using external tune voltages.

All these demonstrator ICs show that despite requiring two separate SDACs, the introduced interleaved architecture is very suitable to design small, low-power DACs with good performance at very high sample rates. 


\section{Contents}

Samenvatting......

Abstract . V

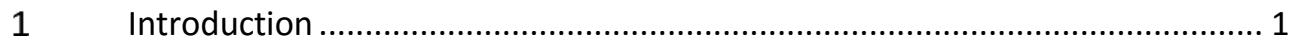

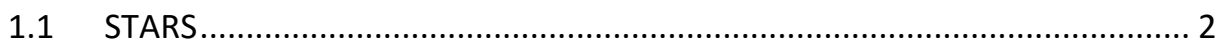

1.1.1 Analog and Digital Performance ......................................................... 4

$1.2 \quad$ Waveforms for Radar Applications …………....................................... 5

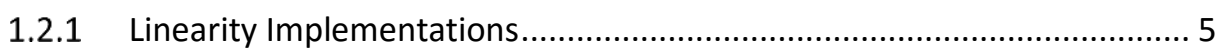

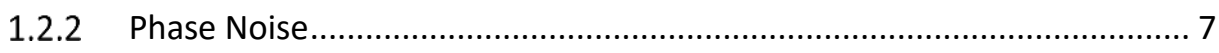

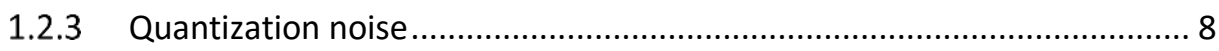

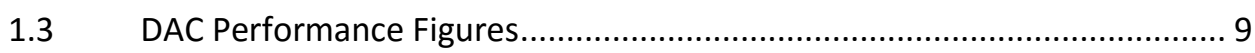

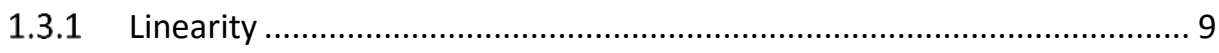

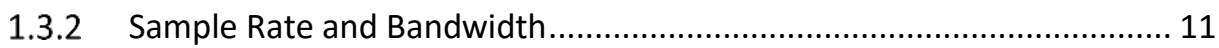

1.3.3 Power Consumption and Area .......................................................... 11

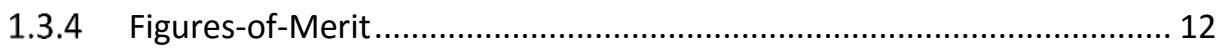

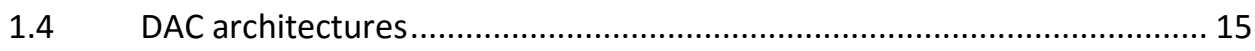

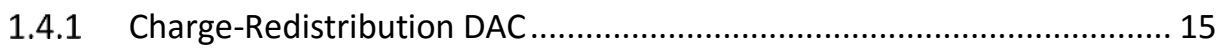

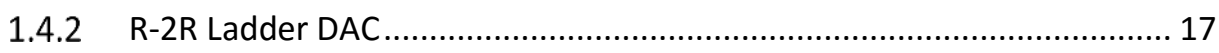

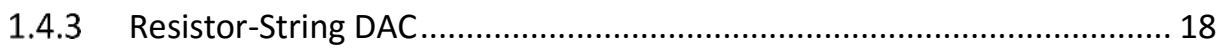

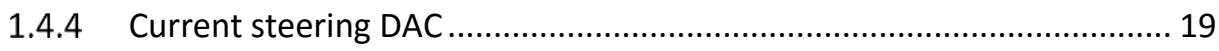

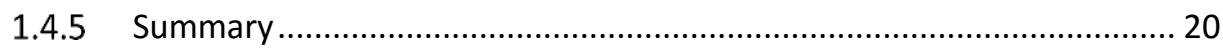

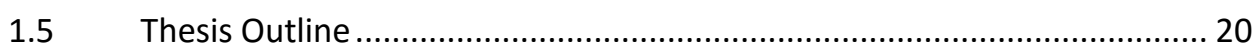

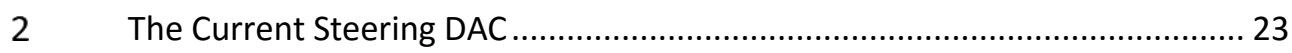

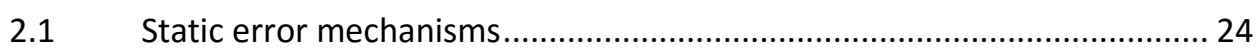

2.1.1 Output resistance .......................................................................... 24 


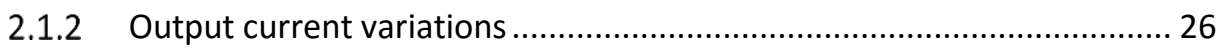

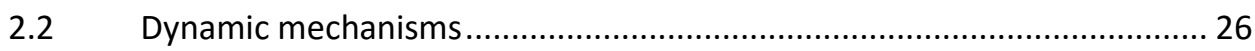

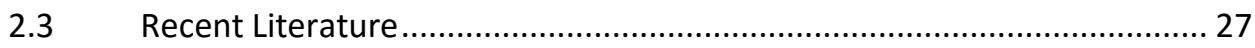

2.3.1 Extra Cascodes with Bleeding Current Sources .................................... 27

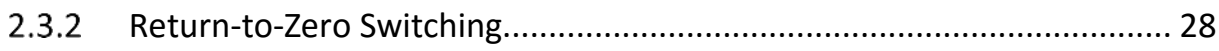

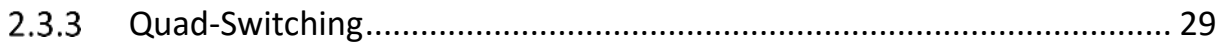

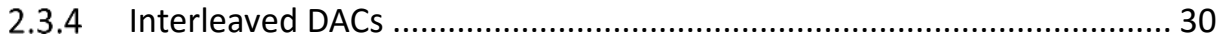

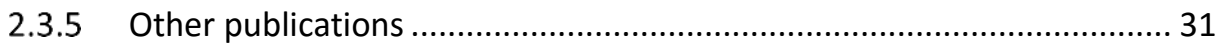

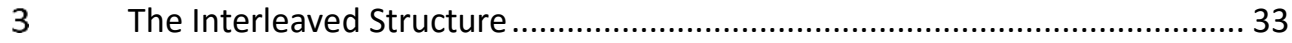

3.1 Interleaving errors mechanisms .......................................................... 35

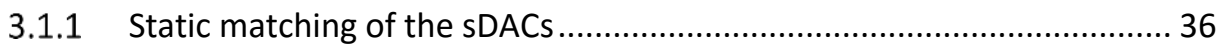

3.1.2 Dynamic matching of the multiplexer .................................................. 39

3.1.3 Multiplexer transistor nonlinearities .................................................... 41

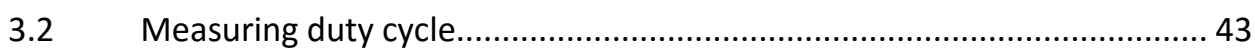

$4 \quad$ Medium Speed Demonstrator: 12-bits at 1.7GS/s ..................................... 45

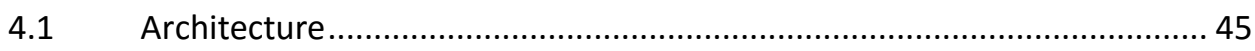

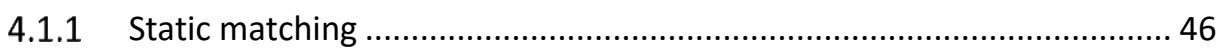

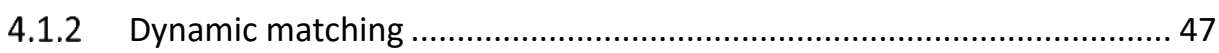

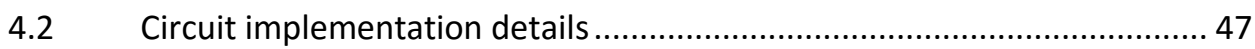

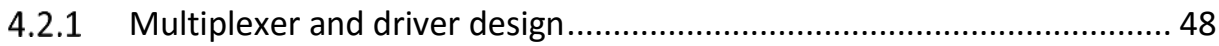

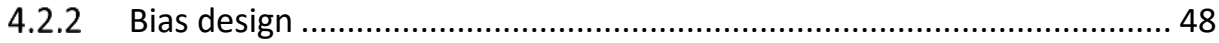

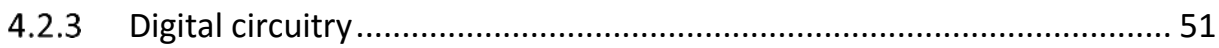

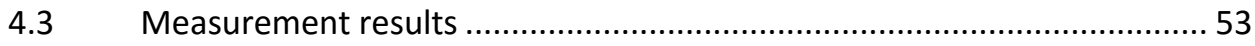

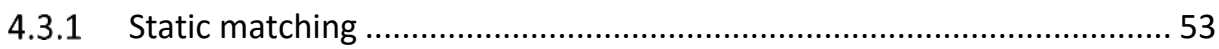

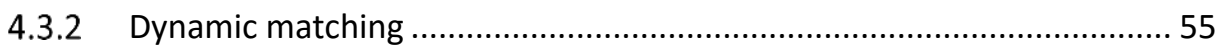

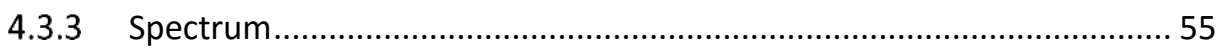

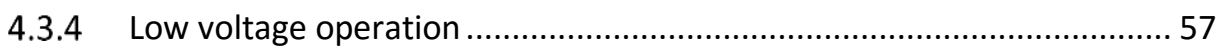

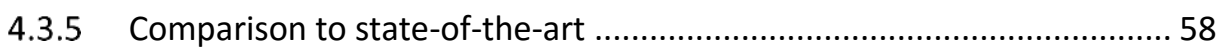

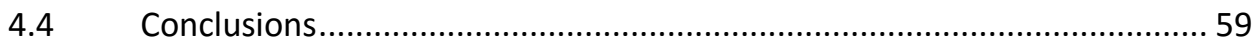

$5 \quad$ High-speed Demonstrator: 9-bits at 8.8GS/s and 11GS/s ............................ 61

5.1 Suppressing code-dependent supply and bias load: Quad-switching ........ 62

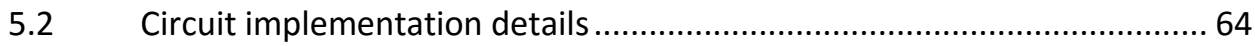

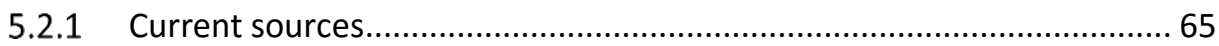




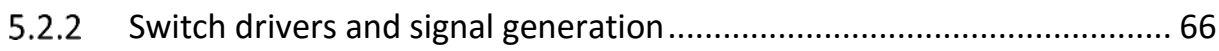

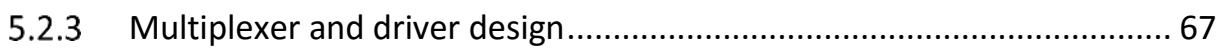

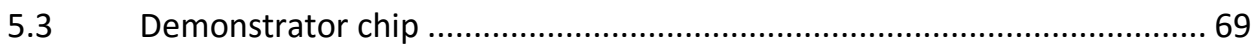

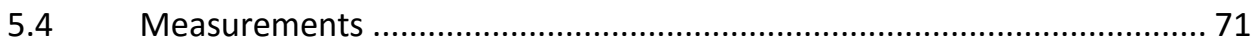

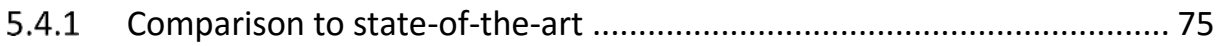

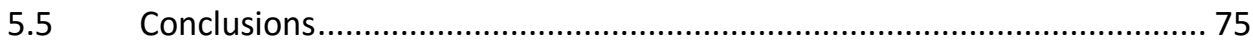

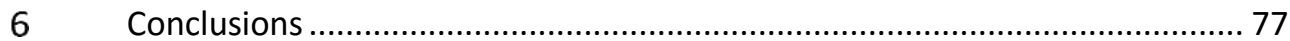

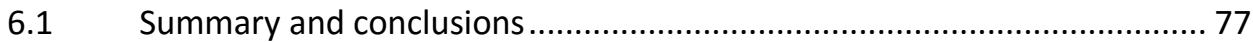

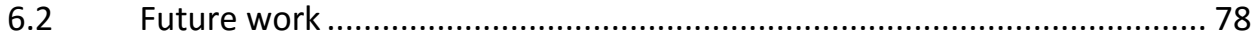

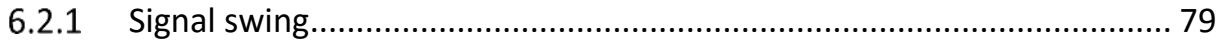

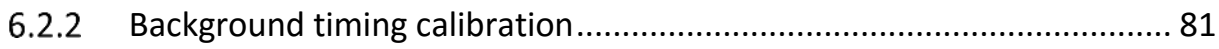

A Co-existing timing and amplitude errors ..................................................... 83

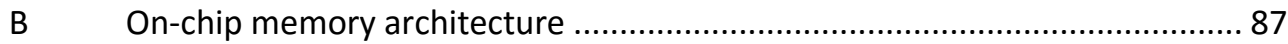

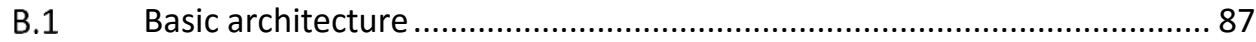

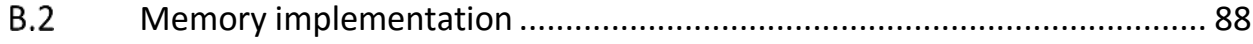

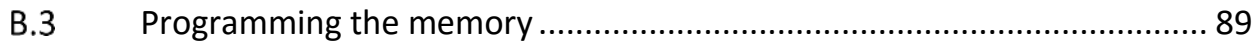

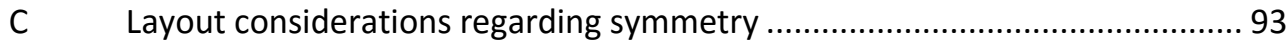

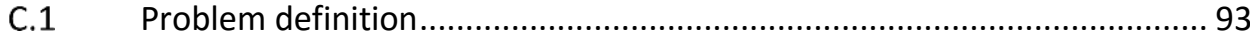

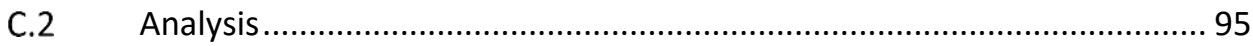

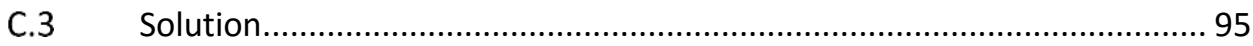

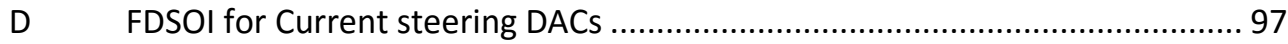

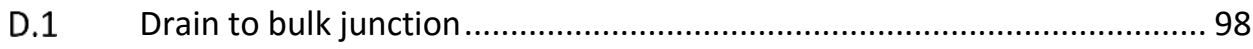

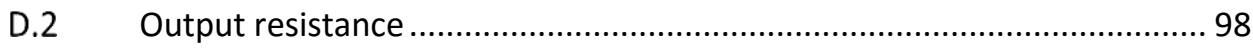

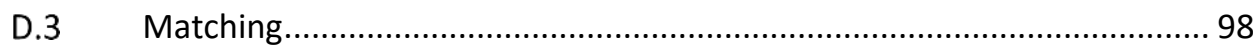

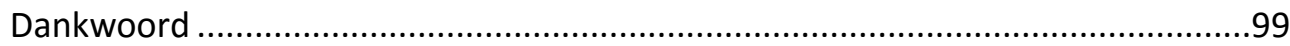

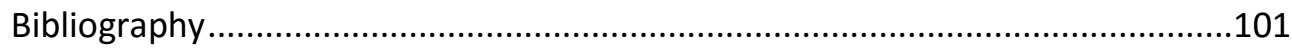

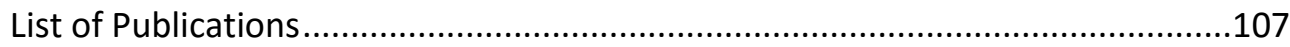

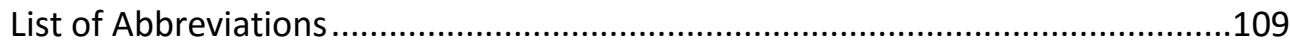





\section{Chapter 1 Introduction}

A digital-to-analog converter (DAC) is a device that converts digital data into an analog signal. For electronic circuits the analog signal is typically in the form of current, voltage or charge.

The digital data represents a signal that is both time and amplitude discrete, while analog signals are both time and amplitude continuous ${ }^{1}$. The spectrum of the digital signal is an infinitely repeating copy of the first Nyquist zone, illustrated by figure 1-1. This can be reproduced in the analog domain by using a (continuous time) impulse function to reconstruct the digital signal. The first problem with this is that it is not possible to make an ideal impulse: even implementing an approximation is quite hard. The other reason not to use ideal(ish) impulse shapes is that generally only the first Nyquist zone is of interest: recreating other Nyquist zones is counterproductive if only the first is relevant.

This desired first Nyquist zone is located between DC and half the sample rate, the Nyquist frequency. Sometimes DACs are used in sub-sampling mode, and a higher zone is used, however this is rare and in this thesis the focus is on regular DACs which are intended to create signals in the first Nyquist zone.

\footnotetext{
${ }^{1}$ Fundamentally, for example in a charge based DAC the amount of charge is a discrete number of charge carriers, while also time might be regarded as discrete. For our requirements however we can treat both as continuous.
} 


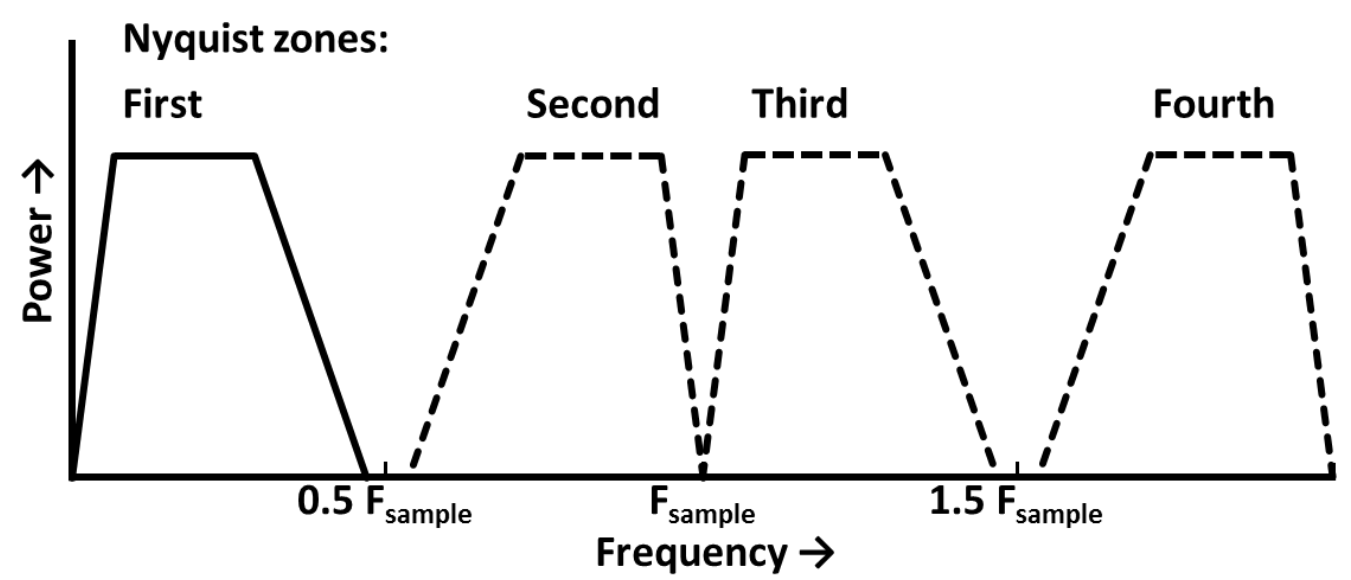

Figure 1-1: Example spectrum of a digital signal

If only the first Nyquist zone is to be reconstructed from the digital signal, a sinc-shaped impulse response would be the preferred solution. Using a sinc, only the first Nyquist zone is recreated. However since a sinc response is non-causal, it requires knowledge about the future or it causes an infinite latency which is respectively not (yet) possible and not useful. Approximating a sinc response is possible, but to do this in the analog domain in a linear way at high frequencies is not a viable option.

Especially for high-speed DACs, generally a $0^{\text {th }}$ order hold function is used: the DAC converts the digital data to an analog output value, and it holds that value until the next clock cycle when new data arrives. This creates a staircase interpretation of the original digital data. In reality the bandwidth is finite, which will smoothen the staircase by suppressing high frequency components. Compared to a sinc response this means that besides the wanted signal in the first Nyquist zone, there are also unwanted signals in higher Nyquist zones. These latter signals can be suppressed by a regular filter. Sometimes higher order hold functions are used, for example a $1^{\text {st }}$ order hold function that interpolates linearly between samples. These higher order hold functions reduce the signal strength for higher frequencies. However in practice it will often be more straightforward to increase sampling speed. This moves the other Nyquist zones further away from the desired signal, reducing demands on the analog post-filter.

\subsection{STARS}

The work presented in this thesis is part of the STARS project. The objective of the STARS project is: "to develop within four years the necessary knowledge and technology 
that can be used as a baseline for the development of reconfigurable sensors and sensor networks applied in the context of the security domain" [1]. In this thesis, DAC architectures are developed for usage in radar transmit frontends.

Most components of a radar system could both be implemented in digital or in analog hardware, which both have specific advantages and disadvantages. From a reconfigurability point-of-view, general purpose digital hardware such as CPUs and FPGAs are inherently capable of being reprogrammed to perform a wide range of tasks. A more dedicated DSP has a more limited number of applications, but within those applications it can still be designed to be reconfigurable. A custom digital ASIC is generally a lot more power and area efficient [2] than more general purpose hardware (under equal conditions) but generally has little options for unforeseen reconfiguration.

The analog equivalent to the highly programmable FPGA is the FPAA (FieldProgrammable Analog Array). FPAAs have been published since the 1990's [3], however commercial applications have been very limited. Currently only one single commercial manufacturer offers just three types of FPAAs. These FPAAs offer $2 \mathrm{MHz}$ bandwidth [4] and are suited to implement non-high performance time invariant analog systems, making it unsuitable for many radar applications.

A flexible analog ASIC can be designed and would for example be capable of wideband operation with flexible filter center frequency and bandwidth. This level of reconfigurability is however not comparable to the degree of flexibility that flexible digital systems can offer. It can be concluded that a true reconfigurable system should perform as many tasks as realistically feasible in the digital domain, and only when there is no other option it should perform specific tasks in the analog domain. A major performance limiting block in such a digital-analog transmit system, that decides how much can be done in the digital domain and what needs to be done in the analog domain is the DAC [5]. To enable as much functionality in the digital domain, and hence to maximize potential reconfigurability, the DAC must be both fast and accurate.

Modern phased array systems contains thousands of separate $T / R$ (transmit/receive) modules. For example the APAR naval radar system uses $13696 \mathrm{~T} / \mathrm{R}$ modules [6]. A flexible radar system would use a DAC in each $T / R$ module close to the antenna, in order to perform as much processing in the digital domain. Due to the number of modules the required DACs should not only be fast and accurate, but also must have a low power consumption. 


\subsubsection{Analog and Digital Performance}

Analog and digital systems have both their specific advantages and disadvantages. Whether an analog or a digital implementation is to be preferred depends on the specific constraints which must be met. A simple low-pass filter with a $20 \mathrm{GHz}$ cut-off frequency is easily designed in the analog domain; a simple RC filter implements this behavior. At the same time, a corresponding digital $20 \mathrm{GHz}$ low-pass filter would be extremely power hungry. The opposite holds for e.g. a very accurate $x 8$ multiplier that can be implemented in the digital domain by a single shift operation, while the analog equivalent would be a lot more complex. There are some basic laws regarding the analog / digital comparison which do give more insight in the tradeoff between choosing analog or digital circuitry.

One of the most fundamental differences between analog and digital signal processing is the SNR to power ratio. To increase the SNR by $6 \mathrm{~dB}$ in a given analog system the dissipated power will also need to be increased by $6 \mathrm{~dB}$ : so a 4 times increase in power consumption. Meanwhile in digital systems, an extra $6 \mathrm{~dB}$ in SNR requires only one more bit [7]. Depending on the used operations, power consumption of a digital system will typically scale somewhere between linear and quadratic with the number of bits. From this it follows that power consumption of analog circuits scales a lot worse than power consumption of digital circuits when high accuracy is required. At the same time when high accuracy is not needed, the power benefit for digital systems is a lot more limited than for analog systems.

The previous paragraph dealt purely with the noise performance. However analog circuits also suffer from non-linear distortion, while digital filters are virtually immune to this.

Another fundamental difference between analog and digital circuits is the predictability. For example an analog filter will always suffer from device spread. Tuning loops can be included to limit the impact of this spread [8], however regardless of device spread digital filters will always perform exactly the function they are designed to perform. This also allows for far more complex digital filters than what is realizable in analog filters [9].

Overall it can be concluded that analog implementations are superior for relative low complexity and accuracy demands and wherever you cannot meet speed requirements in digital, while digital implementations are superior if high complexity and accuracy is 
required. The exact point where this happens depends on the specific implementation requirements and available technology. In addition it also depends largely on the availability and performance of the data converters [5]; specifically for transmitters these data converters are DACs, which are the topic of this thesis.

\subsection{Waveforms for Radar Applications}

For most radar applications the required output waveform is a constant envelope (CE) signal [10]. Compared to systems without a CE, systems with CE can obtain much higher efficiencies due to the ability to employ non-linear power amplifiers [11].

\subsubsection{Linearity Implementations}

CE systems have reduced requirements on linearity since there is no intermodulation distortion which needs to be taken into account. However regular harmonic distortion is still an issue. As mentioned before, the output spectrum of a DAC consists of repeating images. If harmonic components would fall outside the Nyquist band, they will fold back into the Nyquist band where they can distort the wanted signal. This is illustrated by figure 1-2.

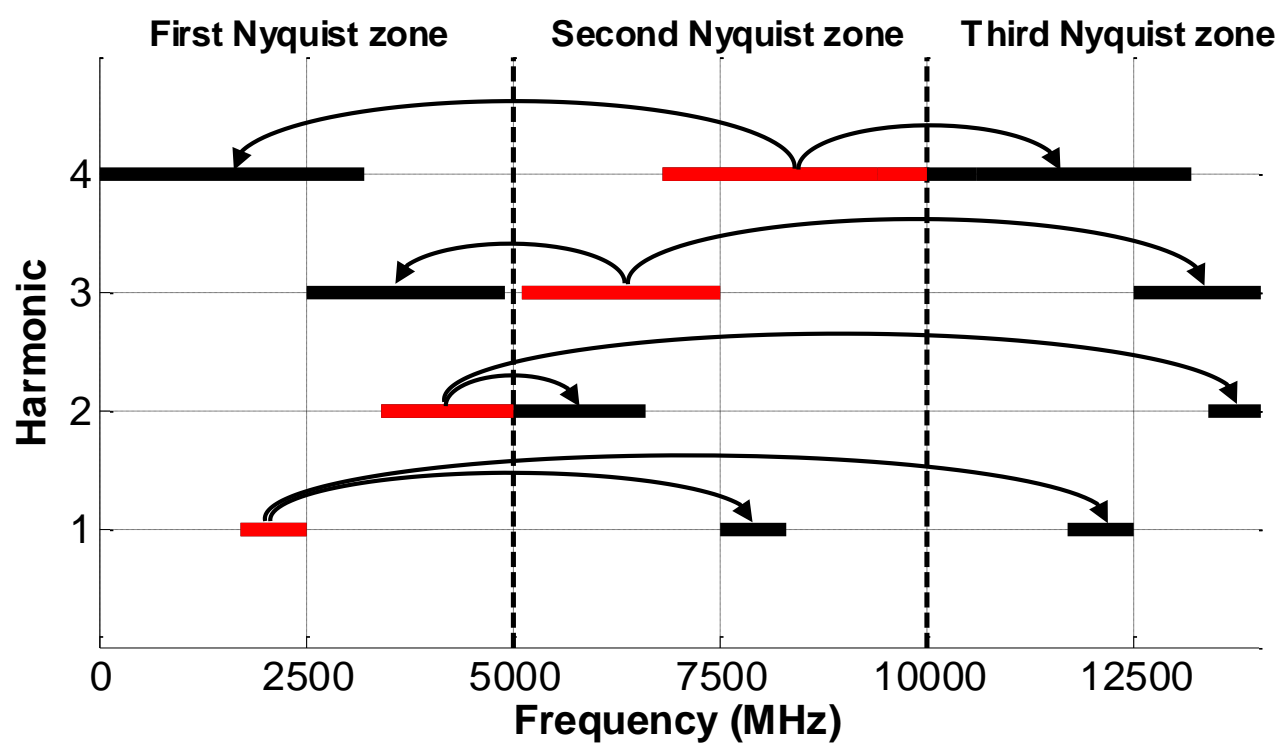

Figure 1-2: Folding of harmonics in a 10GS/s DAC with a 1.7GHz-2.5GHz CE signal: the red lines are the original harmonics, while the black ones are the other frequency bands they fold to 
So while the harmonics will always appear inside the Nyquist band, using proper frequency allocation it can be ensured that the harmonics do not fold into the signal bandwidth.

An example of frequency allocation that can reduce linearity demands is shown in figure 1-3. It represents a $10 \mathrm{GS} / \mathrm{s}$ DAC with a $200 \mathrm{MHz}$ output bandwidth located between 3.5 and $3.7 \mathrm{GHz}$. The figure shows the locations of the different harmonics of the DAC; the first harmonic is the fundamental output. The bandwidth taken by each harmonic is $200 \mathrm{MHz}$ times $n$, where $n$ is the order of the harmonic. This means that evading higher order harmonics becomes progressively harder. However since the magnitude of higher order harmonics will normally be smaller than lower order harmonics this is usually acceptable. In the shown example the first harmonic inside the signal band is the $10^{\text {th }}$ harmonic, the first odd harmonic is the $13^{\text {th }}$ harmonic. This will give an SFDR inside the signal band which is a lot better than the complete Nyquist performance. The number of harmonics that can be kept outside the signal band depends on the bandwidth and frequency planning. In general a higher Nyquist bandwidth allows for more space, which allows for better frequency planning and less harmonics that end up in the signal band.

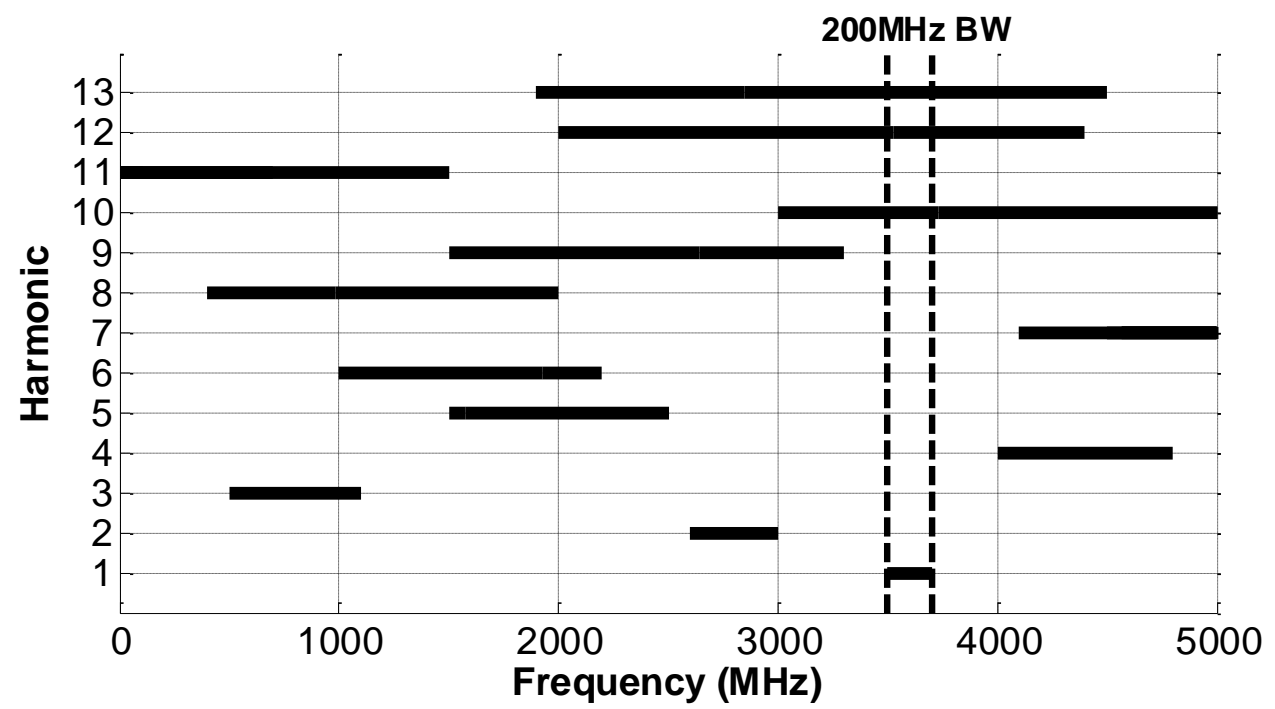

Figure 1-3: Harmonics locations of a $10 \mathrm{GS} / \mathrm{s}$ DAC with $200 \mathrm{MHz}$ signal bandwidth and $\mathrm{n} * 200 \mathrm{MHz}$ bandwidth for each harmonic 
The DAC will also generate relative strong image frequencies outside the Nyquist band. A band pass filter would be required to pass the wanted signal band while blocking the unwanted harmonics and image frequencies outside the signal band.

\subsubsection{Phase Noise}

An important requirement of generated signals for the use in radar systems is that the phase noise of the generated signal must be low. A widely used signal in radar systems is a chirp which is a sine wave with increasing frequency. Chirps can be generated by DACs or by PLLs [12]. Comparing chirp generating DACs and PLLs in terms of e.g. phase noise is not directly possible, since a PLL has a low frequency input which it multiplies to get the high output frequency, while a DAC has a high frequency input that it divides down to get a lower output frequency. So a DAC would require also a PLL to first generate a (stable) high frequency clock input, which it can divide down to generate the required chirp. An advantage for a DAC-based chirp generator is that a single high performance stable clock can be shared by many DACs.

Although (phase) noise performance was not a design consideration for the DACs presented in the thesis, a basic comparison can still be made with PLL systems. The PLL design from [12] is optimized for generating fast chirps, something a high-speed DAC would also be able to generate. The design in [12] achieved a phase noise of $-92 \mathrm{dBc} / \mathrm{Hz}$ at a $1 \mathrm{MHz}$ offset with a carrier frequency of $4 \mathrm{GHz}$.

The phase noise of the $28 \mathrm{~nm}$ FDSOI DAC presented in chapter 5 of this thesis, measured at a $5 \mathrm{GHz}$ output frequency using a spectrum analyzer is shown in figure 1-4. Below $100 \mathrm{kHz}$, in this measurement the phase noise was dominated by the spectrum analyzer noise and clock generator noise. The phase noise at $1 \mathrm{MHz}$ offset for the $5 \mathrm{GHz}$ carrier is $-130 \mathrm{dBc} / \mathrm{Hz}$.

These measurements should not be taken as indication that a DAC will have superior noise performance compared to a PLL setup: a low phase noise PLL will still be required to generate the stable high frequency clock for the DAC. Because for such a PLL there are for example no requirements on settling speed and output bandwidth, better phase noise is expected compared to a chirp generating PLL. These results indicate that a chirp generating DAC may have a negligibly small impact on overall phase noise of radar systems. 


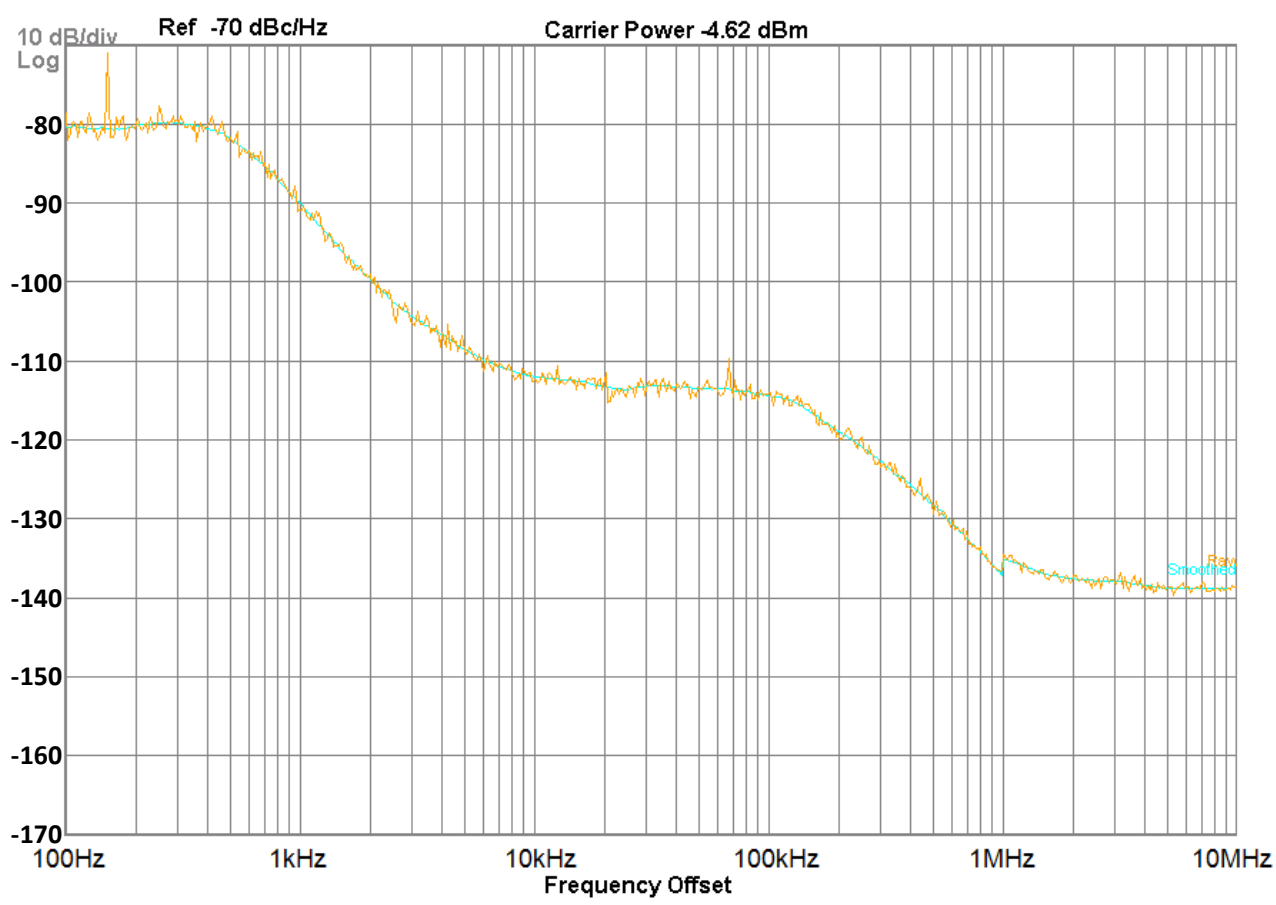

Figure 1-4: Measured phase noise at $5 \mathrm{GHz}$ carrier using $28 \mathrm{~nm} 11 \mathrm{GS} / \mathrm{s}$ DAC

\subsubsection{Quantization noise}

Quantization noise is the noise-like error generated due the finite number of bits. This error is spread out across the entire Nyquist band. If only part of the Nyquist band is used for the signal, the system is oversampling. In this situation the effective quantization noise in the signal band is reduced: every factor of four larger Nyquist bandwidth compared to the signal bandwidth results in effectively an extra bit for noise performance [13]. When the required number of bits is due to demands on the quantization noise of the converter, this means that with increasing oversampling ratio a lower number of bits is required while still meeting the required (in band) noise density.

Besides the noise performance, also the obtainable linearity is dependent on the number of bits [14]. Additionally adding/removing an LSB generally has little influence on total power consumption; the small extra current and switches require little (drive) power. 


\subsection{DAC Performance Figures}

The goal of a DAC is to reproduce the digital input signal in the analog domain. Therefore main performance indicating figures represent a measure on the accuracy of the reproduction of the digital signal in the analog domain. Noise on the output signal is one aspect, this can both be 'normal' noise on the output signal, including quantization noise, and phase noise in the clock circuitry. Generally the overall performance of a high-speed DAC will not be limited by noise. The typically used architectures produce inherently little noise while due to demands on the delivered output power the signal levels are high, resulting in a large signal-to-noise ratio.

\subsubsection{Linearity}

The DACs presented in this thesis are mainly optimized for high-speed and linearity. Ideally the conversion from the digital domain to the analog domain is perfectly linear; in practice this is never the case. Nonlinearities give rise to harmonic distortion tones in the output, as described in section 1.2.1, even if these would appear to fall outside the Nyquist bandwidth, they fold back inside the Nyquist zone. Additionally there might be non-harmonic spurs in the output signal of the DAC. These non-harmonic spurs either have no relation to the fundamental frequency, such as a spur due to another clock in the system, or which have a different relation to the fundamental than a regular harmonic has.

The linearity and spur performance can be quantified in two ways. The first way is intermodulation performance. In this, two digital sine waves with close together frequencies that have a fixed frequency spacing are generated, each with half the full scale amplitude. These sine waves are digitally added and used as input for the DAC. In the analog output spectrum of the DAC, besides the two input tones this creates also IM3 tones, as shown in figure 1-5. The difference between the power in the wanted tones and the generally dominant third order intermodulation products can be calculated for different signal frequencies, resulting in an IM3 versus frequency graph.

A second performance indicator is the spurious-free-dynamic-range (SFDR). For this a single, full scale, digital sine wave is generated and used as input for the DAC. In the resulting analog output spectrum of the DAC the difference between the power in this tone and the largest spurious tone is taken. This takes not only harmonics, but also other possible spurs into account and gives an SFDR value for each signal frequency. It is important to explicitly take the bandwidth where the spurious tones must reside into 
account. An often used approach, which is also used in the rest of this thesis, is to take every spur in the entire used Nyquist zone into account. However sometimes only part of the Nyquist bandwidth is taken into account, which disregards any signal outside that part of the Nyquist band and which therefore results in a more optimistic SFDR [15].

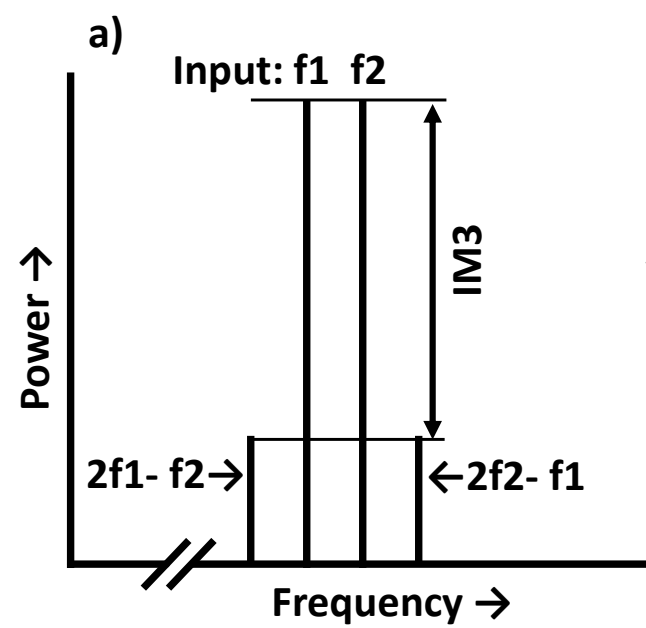

b)

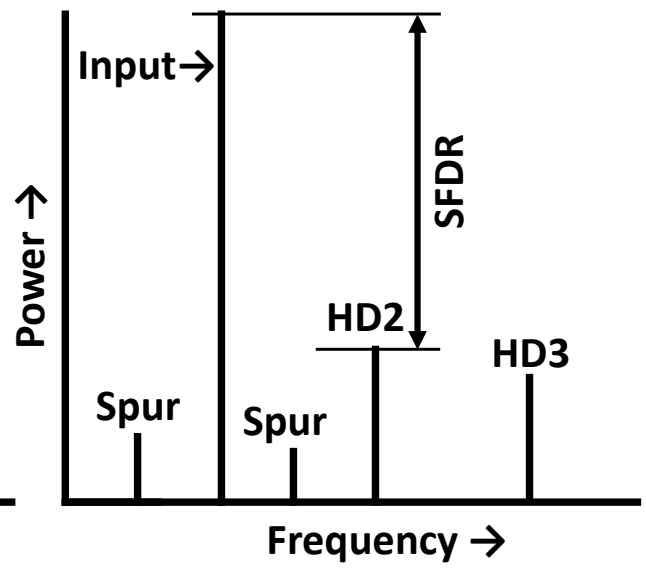

Figure 1-5: a) Spectrum with IM3 definition, b) spectrum with SFDR definition

\subsubsection{DNL and INL}

A widely used metric to describe DC linearity in DACs is the Differential-Non-Linearity (DNL) and Integral-Non-Linearity. The DNL is the difference between the size of an actual output step and its ideal value. The INL is the difference between the sum of all previous output steps and the corresponding ideal value. Figure 1-6 illustrates these definitions. It should be noted that while for clarity a staircase is drawn, in reality noninteger digital input codes do not exist, so also no corresponding analog output value exists. Generally the INL and DNL are described using a single number by taking the maximum value of all steps as the reported DNL/INL values.

In this thesis the DNL and INL are not a goal but a method: the goal is to achieve a sufficiently high SFDR and to do this the DNL and INL inherently need to be sufficiently low. 


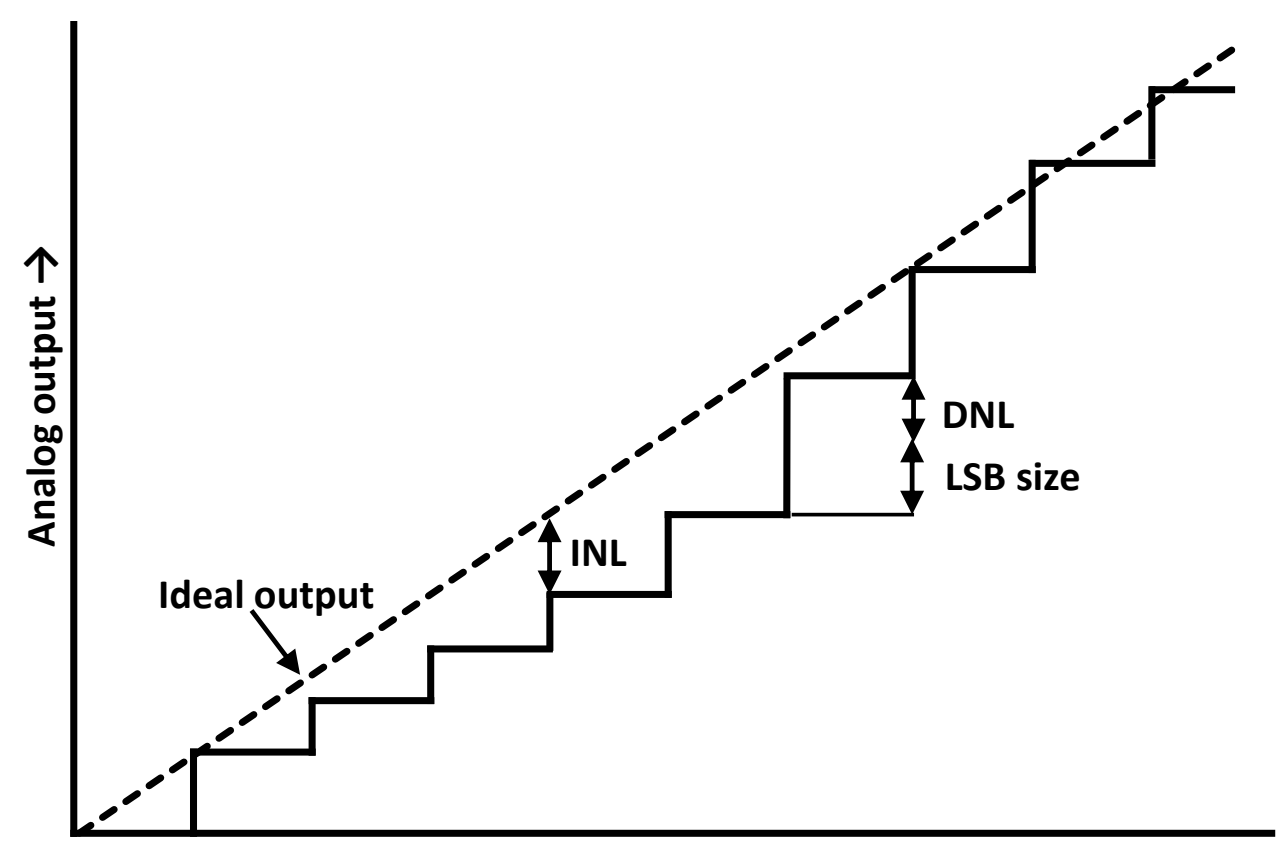

\section{Digital input code $\rightarrow$}

Figure 1-6: DNL/INL definition

\subsubsection{Sample Rate and Bandwidth}

Besides good accuracy the second requirement of a high-speed DAC is that it is fast. The speed of a DAC is specified in terms of both analog signal bandwidth and the sample rate of the converter. The signal bandwidth of a regular, non-undersampling, converter can at most be equal to the Nyquist bandwidth: half the sampling frequency. At a fixed signal bandwidth, a higher sample frequency can be beneficial: image frequencies are moved further away from the signal band, thereby reducing demands on filtering, see also the introduction of this chapter.

\subsubsection{Power Consumption and Area}

A certain amount of power and area will be used to obtain the given sample speed and accuracy. Low power consumption is essential for many applications. For mobile applications it increases battery life and in general it reduces requirements on cooling.

The cost of an IC is directly dependent on the area used. A small chip area implies that it is cheap and it also makes integration with other circuitry easier. 


\subsubsection{Figures-of-Merit}

To aid comparisons between different architectures a FoM (Figure-of-Merit) can help. A FoM aims at simplifying the relations between different parameters and combine it into a single number which can be compared with competing designs. For example generally if you double the power consumption, the SNR (Signal-to-Noise Ratio) will improve with $3 \mathrm{~dB}$ in a typical analog circuit. This implies that a design with $3 \mathrm{~dB}$ better SNR, but four times the power as another design, is a worse design, assuming everything else is equal. In practice everything else will not be equal, and a design with worse FoM can still have superior performance in other areas. Still a good FoM is a useful tool.

The usefulness of FOMs is illustrated by the related field of ADCs (Analog-to-Digital Converters), where designs are compared based on their FoM. Generally for low resolution ADCs (<10 bit) Walden's FoM [16](1-1) is used, while higher resolution ADCs, which are more often thermal noise limited, use a modification of Schreier's FoM [17] which also takes distortion into account [18](1-2). The main difference between the used FoMs is if energy scales with a factor of two per extra effective bit, or with a factor of four.

$$
\begin{gathered}
\mathrm{FOM}_{\text {Walden }}=\frac{P}{2^{E N O B} * f_{S}} \\
\mathrm{FOM}_{\text {Schreier-modified }}=S N D R+10 \log _{10}\left(\frac{B W}{P}\right)
\end{gathered}
$$

Here $P$ is the consumed power, ENOB the effective number of bits, $f_{s}$ the sampling rate, $S N D R$ the Signal-to-Noise-and-Distortion Ratio and $B W$ the Nyquist bandwidth.

In contrast to the ADC field, in DACs the usage of FoMs is much more limited, and they are not used in this thesis. ADCs are often largely noise limited in performance, however high-speed DACs are mainly limited by distortion. While a clear tradeoff between power and noise performance can generally be found, there is no clear tradeoff between power and linearity. This is further complicated when clock speed/Nyquist bandwidth is added to the equation. In a noise limited system where the majority of the power consumption is dynamic (scaling with clock speed), halving the clock speed will also cut power consumption with a factor two while keeping the other metrics largely equal. However even if we assume power consumption of a DAC is mainly dynamic, which 
often is not the case, halving their clock frequency will also have a large effect on their linearity.

Despite this various FoMs have been suggested for DACs, although they have never received widespread acceptation. Below some of proposed FoMs are briefly discussed.

\section{FoM \#1}

A FoM which is for example used in [19], and which is seen in publications on very highspeed DACs (10-40GS/s) is given by

$$
F O M=\frac{P}{2^{N} f_{\text {sample }}} .
$$

In this equation, $P$ is the power consumption of the circuit, $N$ the number of bits, and $f_{\text {sample }}$ the sampling frequency of the system. While some of the designs in this thesis would do very well when this FoM is taken into account, it is not a very useful one. Since the accuracy of the conversion is ignored, the best FoM would be achieved by disabling the IC. Clearly accuracy needs to be taken into account to have a useful FoM.

\section{FoM \#2}

Another known FoM was introduced in [20] as

$$
F o M=\frac{V_{\text {swing }} f_{\text {sig }}}{P} 10^{\frac{S F D R[d B]}{20}}
$$

in which $V_{\text {swing }}$ is the swing at the output of the DAC and $f_{\text {sig }}$ is the frequency of the used test tone. This FoM does take the linearity into account in the form of the SFDR. However the output swing of a current DAC can easily be increased by for example using a (more) high-ohmic load resistance, at which point it depends on the dominant distortion mechanism whether the SFDR would decrease. As alternative the load resistance could be increased while the output current is decreased, which should lower power consumption while not changing any of the other parameters. In addition it assumes that with doubling the power the obtainable signal frequency at a given swing and SFDR can also be doubled, which is questionable. 


\section{FoM \#3}

In [21] another FoM is given by:

$$
F O M=\frac{2^{\text {ENOB }_{D C} 2^{E N O B_{N y q}} f_{\text {sample }}}}{P_{\text {total }}-P_{\text {load }}}
$$

In this equation $E N O B_{D C}$ is the effective number of bits at DC, and $E N O B_{N y q}$ is the effective number of bits at Nyquist. Furthermore it subtracts the power delivered to the output from the power consumption, which results in a more fair estimation of the actually dissipated power. But the advantage of including the DC performance is not clear: generally the worst-case performance is what should be taken into account for a system, and a typical high-speed DAC is never intended to have an exceptional DC performance. Placing an audio DAC in parallel to the actual DAC, which would then be used for only low frequency signals would result in a very good FoM, while it obviously is not a useful setup. In addition it also assumes that doubling the sample rate at equal ENOB would require only a doubling of power consumption.

\section{FoM \#4}

Two related FoMs are used in [22]:

$$
\begin{aligned}
& \text { FoM }=\frac{2^{N} B W_{70 d B}}{P} \\
& F O M=\frac{2^{N} B W_{70 d B}}{P * A}
\end{aligned}
$$

The $B W_{70 d B}$ is the bandwidth where the SFDR is more than $70 \mathrm{~dB}$, and $A$ is the core area. Taking the area into account is an improvement, although it is not clear why this specific relation would hold across different designs. Using the $70 \mathrm{~dB}$ bandwidth (or another number) is advantageous for a design with a linearity slightly over $70 \mathrm{~dB}$ across a large frequency range, while it would severely penalize designs which are slightly below $70 \mathrm{~dB}$. Such a metric could be appropriate if there was a clear relationship between signal frequency and SFDR. However since this is not the case for many designs it is not a good performance parameter.

\section{Conclusion on DAC FOMs}

Despite all the problems mentioned on existing FOMs, there were many attempts to introduce a suitable FoM for (high-speed) DACs. A FoM will never take every relevant parameter into account and it will also never be a perfectly fair comparison, doing that 
with a single number is not a realistic expectation. So when evaluating proposed FoMs it should be taken into account that it will not and is not intended to be a perfect representation of the overall performance of a design. However the FoMs discussed here either lack important performance figures, or they do not accurately model relationships between the performance figures. Also for all the FoMs discussed here, neither empirical nor fundamental evidence has been presented to support the relationships between different parameters that they assume exist has been shown.

For these reasons in this thesis no FoMs are used to compare performance. Instead the raw performance figures are listed and compared directly.

\subsection{DAC architectures}

Many different architectures that are capable of implementing digital-to-analog conversions are known. Since the focus in this thesis is on DACs for RF applications, only DAC architectures that are capable of high-speed operation are discussed. [23, 24]

Different oversampling and noise-shaping techniques are not discussed here; while these architectures are very capable of generating good performance at lower signal bandwidths, due to their oversampling nature they are not suitable for the signal frequencies required in this research.

\subsubsection{Charge-Redistribution DAC}

The charge-redistribution DAC is found in two flavors: it can be implemented in a serial and in a parallel fashion. The serial implementation requires a clock cycle per bit, while the parallel version can do an entire conversion in a single cycle.

Figure 1-7 shows an example of a parallel charge-redistribution DAC. It consists of an array of binary scaled capacitors. Initially these are all charged to a common voltage, after which the bottom plates of the capacitors can be switched to a reference voltage. Depending on the size of the switched capacitor, this causes a redistribution of the charge, and generates an output voltage which depends on the digital code [25]. 


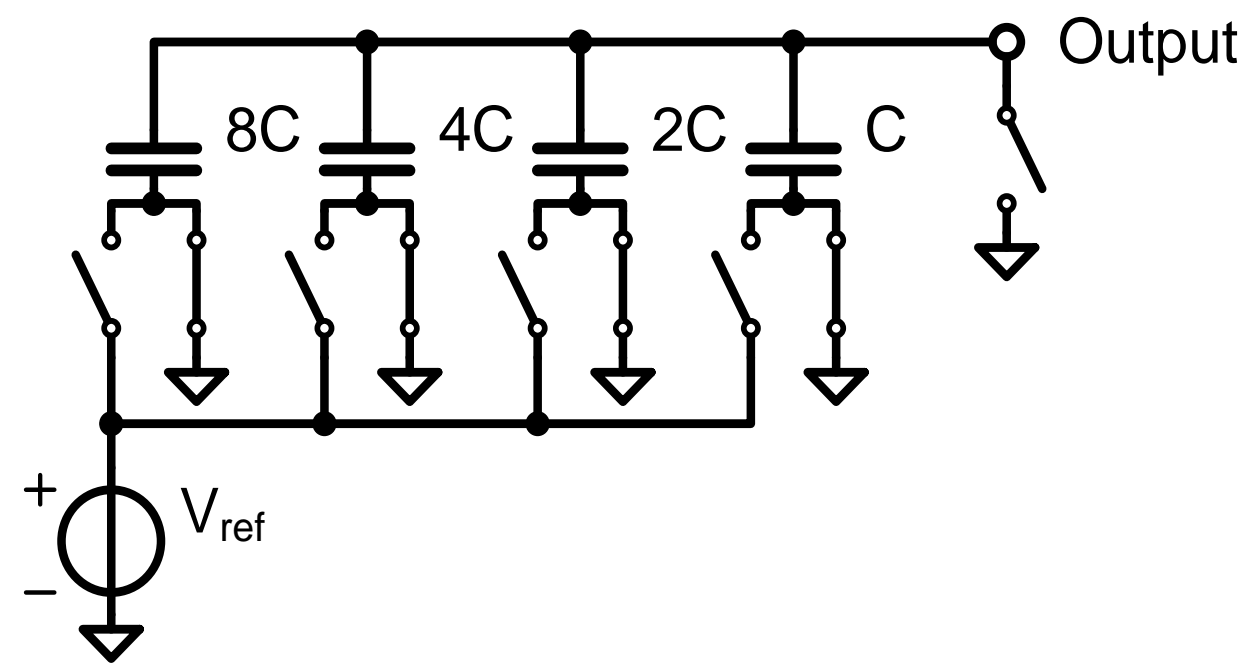

Figure 1-7: Parallel charge-redistribution DAC

The serial charge-redistribution DAC consists of only two equal sized capacitors, and is an LSB-first multi-step architecture. The first half of each cycle the two capacitors are disconnected, and one of them is either charged to a reference voltage, or discharged to ground. The second half the two capacitors are connected, allowing the charge to be redistributed with the charge on the other capacitor which is from the previous steps [26]. While it requires fewer capacitors than the parallel version requires, the matching demands are similar, so the capacitor area required is also similar. The serial implementation is more flexible, but due to its much lower speed generally the parallel version is preferred.

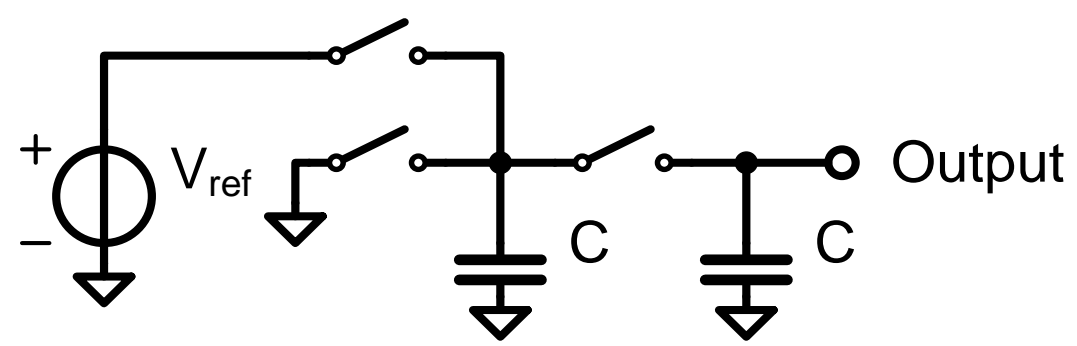

Figure 1-8: Serial charge-redistribution DAC 
These DACs are often used in SAR (Successive-Approximation-Register) ADCs, where they are directly connected to a comparator. For most other applications a buffer would be required after the capacitors.

\subsubsection{R-2R Ladder DAC}

The $R-2 R$ architecture uses a number of resistors, each sized as either ' $R$ ', or two-times ' $R$ ', to generate an output voltage. It can be employed both in a voltage-mode, as shown in figure 1-9, or in current-mode, where the ' $2 R$ ' resistors are connected to ground at the bottom, and a current source at the top injects current, shown in figure 1-10. The difference is weather a voltage or a current is switched. The output impedance of the DAC is always $R$, so when sufficiently low resistors are used it can be directly interfaced to an external $50 \Omega$ reference [27].

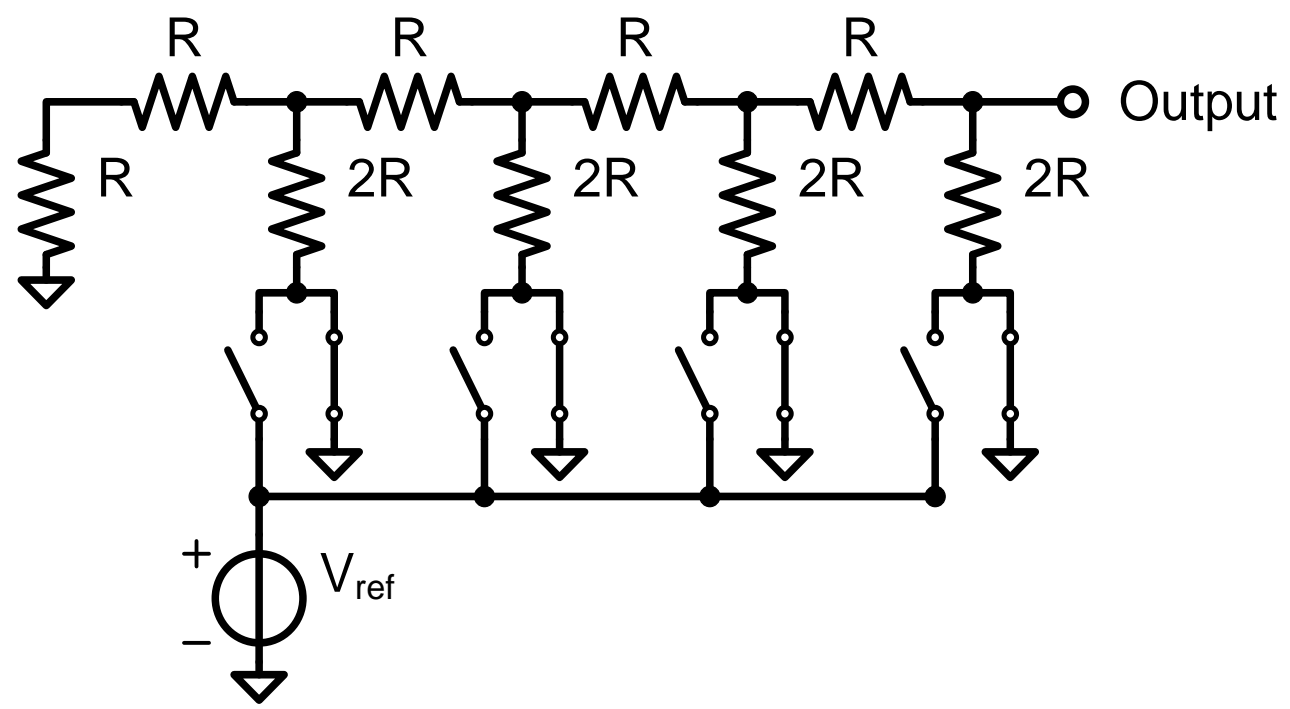

Figure 1-9: Voltage mode R-2R DAC 


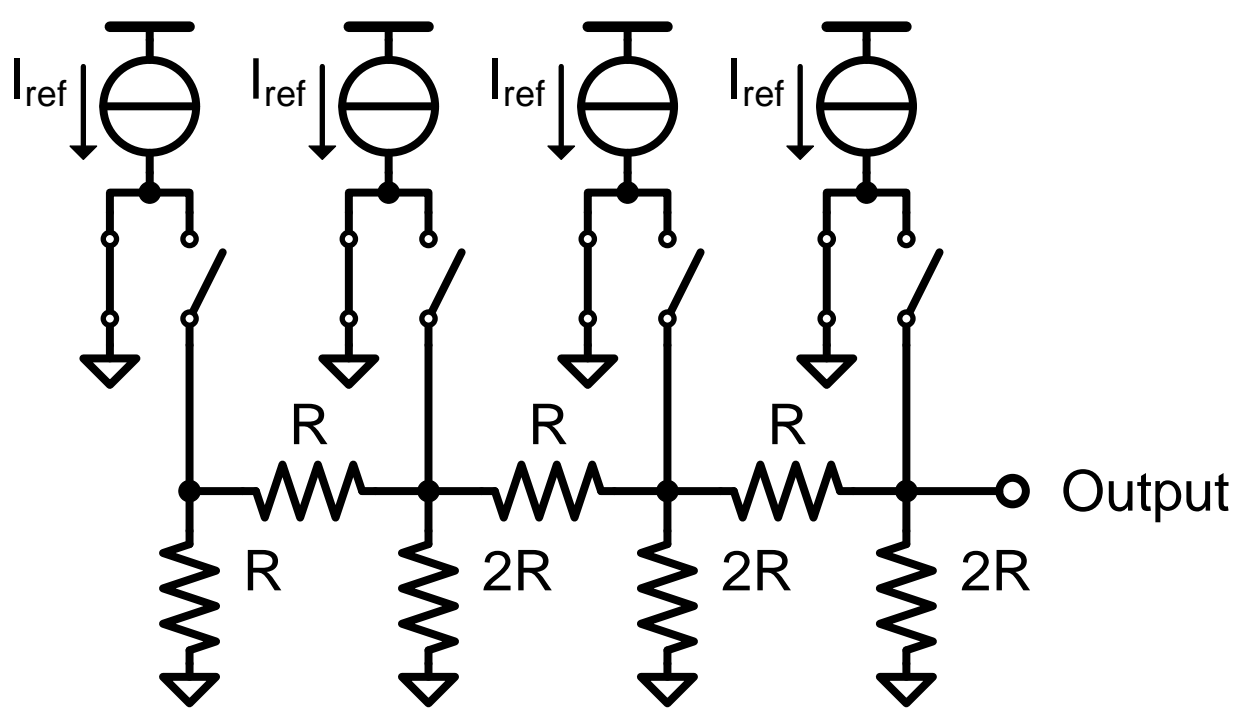

Figure 1-10: Current mode R-2R DAC

However if low-ohmic resistors are used, the load on the reference voltage is high, which may result in distortion products. The current-mode implementation does not suffer from this, however compared to a current steering DAC it has similar limitations, and in addition to that, the majority of the delivered current is wasted. In practice the R-2R DAC is often used in bipolar current steering DACs: minimum sized BJTs require a lot more current than minimum sized MOSTs to properly operate. In order to keep the LSB current reasonable, an R-2R DAC can be used on top of the LSB current sources to perform effectively a current-division [28].

\subsubsection{Resistor-String DAC}

An alternative resistor based DAC is the resistor-string DAC. It consists of a string of resistors between two reference voltages, and an analog multiplexer which selects one of the taps as output [29]. The main advantage of this DAC type is that it is inherently monotonous and will have a very low DNL compared to other DAC types. However a buffer is required since the impedance seen by the output depends on the used tap. Even with a buffer the high frequency performance is limited due to the resulting codedependent RC times, and at higher resolutions the number of resistors required explodes. 


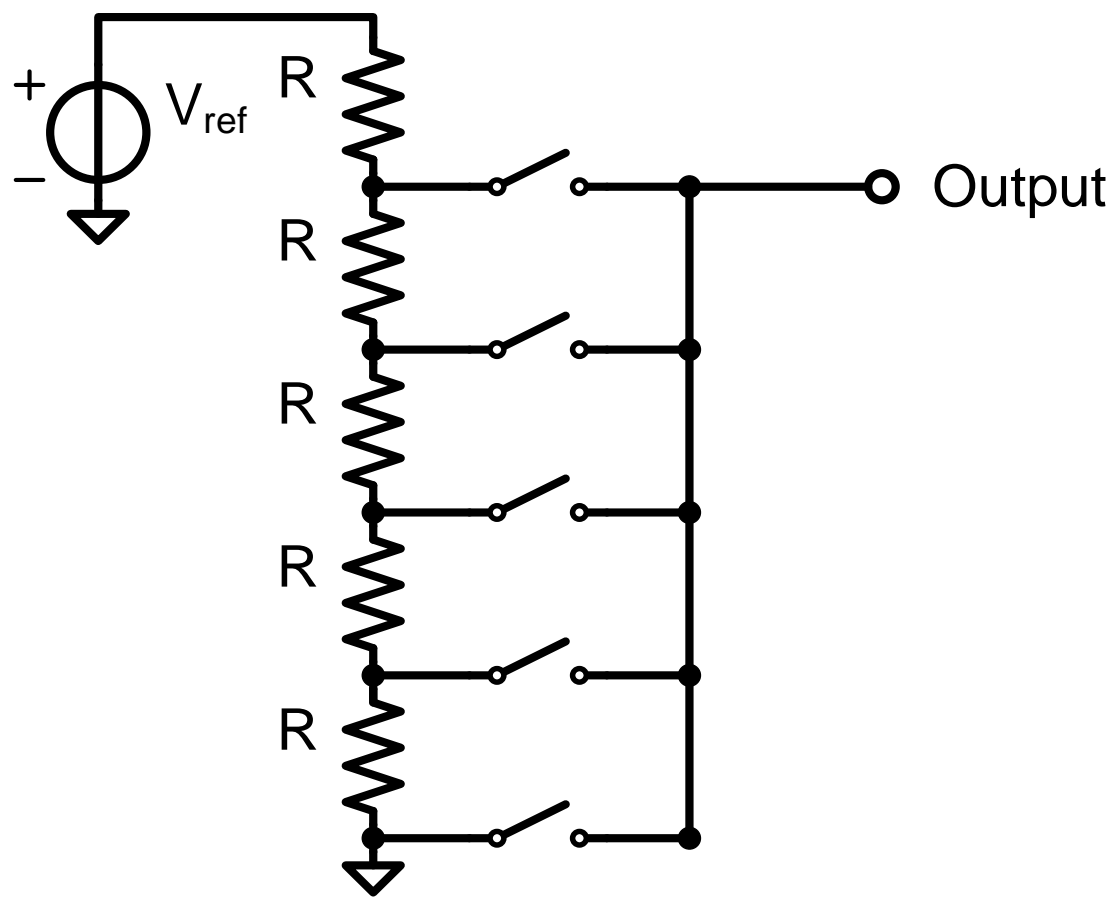

Figure 1-11: Resistor-String DAC

\subsubsection{Current Steering DAC}

The CS (Current Steering) architecture consists of an array of current sources with switches on top of them which can direct the current either to the positive or the negative output. This output current can be directly connected to a resistive load, for example $50 \Omega$, to generate an output voltage. The accuracy at low frequency is mainly limited by the performance of the current sources, while at higher frequencies also the switch performance plays an important role [30]. Since there is no buffer required and switching currents can be done very fast, it is the most popular solution for high-speed DACs. 


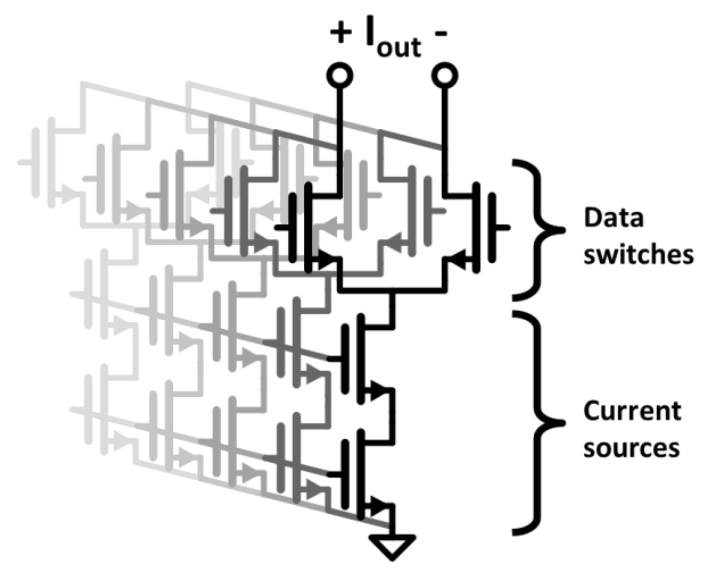

Figure 1-12: Current steering DAC

\subsubsection{Summary}

For high-speed operation the most suitable architectures are those who do not require an extra buffer, since this buffer will limit the performance of the DAC. With this requirement there are two options: The R-2R DAC, which can either be used in voltagemode or in current-mode, and the current steering DAC. Since the voltage-mode R-2R DAC has a high load on the reference node it is less suitable.

While the current-mode R-2R DAC is a useful option to implement the LSB sources in a bipolar process, in a CMOS process with its much smaller transistors this is not an issue. Both require an array of current sources, only the R-2R option only delivers a fraction of the current to the output and has extra potential error sources due to the resistor network. This makes the current steering solution the best option for a high-speed DAC in CMOS.

\subsection{Thesis Outline}

In the next chapter the basics of current steering DACs are discussed. Its operating principles and its limitations are discussed. This is followed by a review of known methods from literature to improve CS DAC performance. Chapter 3 presents an alternative approach to improve DAC performance by dealing with error mechanisms discussed in chapter 2: the interleaved current steering DAC. The advantages and disadvantages of this interleaved architecture are considered, including solutions to solve problems inherent to an interleaved architecture. 
In chapter 4 a practical implementation of the interleaved DAC architecture proposed in chapter 3 is presented. Circuit solutions to deal with limitations of the used architecture are shown, followed by measurement results obtained with the designed chip. The last part of this chapter summarizes the suitability of the interleaved DAC architecture for high-speed signal generation.

While the presented interleaved DAC architecture suppresses many of the spurs generated by regular DACs, it is not able to nullify all of them. At high-speeds especially data-dependent load on the bias and power supply lines is still problematic. Chapter 5 gives an in-depth analysis of these issues and proposes a solution to reduce their impact. It is followed by an implementation of the solution in a designed DAC and the performance obtained by that DAC.

Finally chapter 6 summarizes the results presented in this thesis and presents the conclusions, followed by recommendations to further improve the performance of high-speed DACs. 



\section{Chapter 2 \\ The Current Steering DAC}

The subject of this thesis is the design of high-speed DACs. As argued in chapter 1 , the best suited conventional DACs for this application are the current steering (CS) digitalto-analog converters (DACs). These are commonly used to generate high-frequency signals, and they consist of an array of current sources and current-switches as depicted in figure 2-1. Depending on the digital code, current is switched either to the positive or the negative output.

Since the current switches only are required to redirect the fixed current generated by their corresponding current source, this can be done both fast and accurately. The output current can often be fed directly into a $50 \Omega$ load, removing the need for a buffer that would both be power hungry and would introduce additional non-linearities.

Distortion components in the DAC's output current are due to both static and dynamic error mechanisms. Static errors include those due to mismatch between current sources and those due to the finite output resistance of the current sources. Dynamic errors are due to e.g. timing errors at the switching moment, glitches of the switches and output capacitance of the current sources. High-speed DACs are typically limited in their linearity by dynamic errors; static errors can generally be sufficiently suppressed to not limit the high frequency performance. 


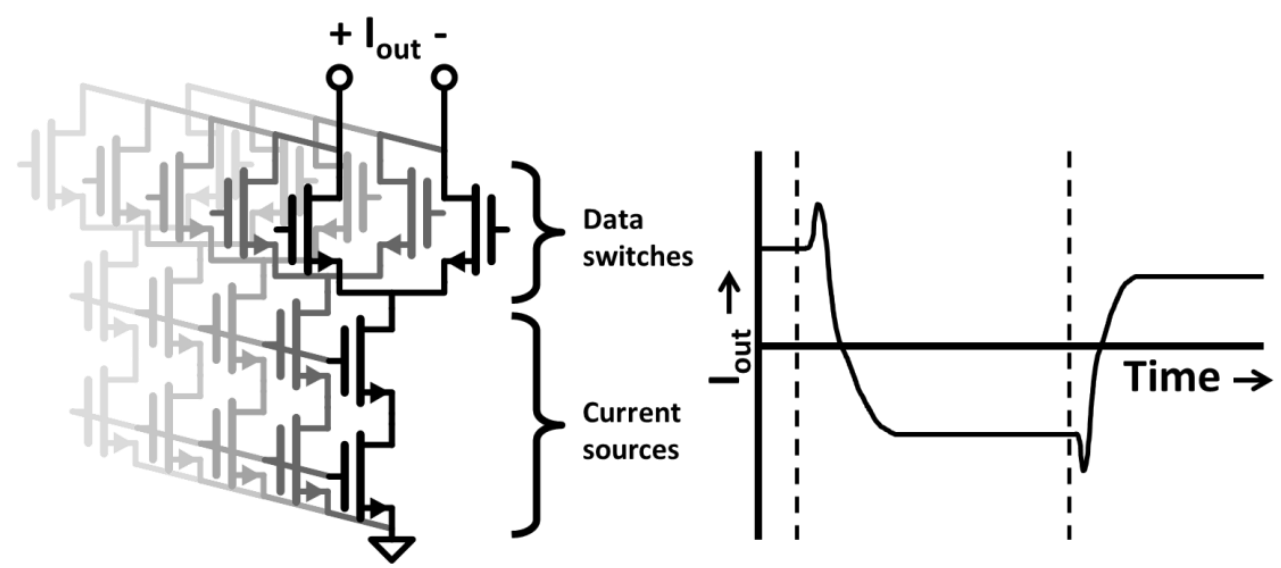

Figure 2-1: Current steering DAC

\subsection{Static error mechanisms}

Static errors in CS DACs are mainly due to non-perfect current sources. An ideal current source has exactly the correct current output and hence has an infinite output impedance. However both of these properties are not satisfied for a real current source realized using mosfets. The following two section discuss in some detail these two nonidealities. For the output impedance only the real part of the output impedance, the output resistance, is taken into account because the imaginary part results in a dynamic error.

The two mechanisms described above are usually dominant. Other errors sources that can be made sufficiently small include layout issues. For example in the layout especially the impedance of the ground connection of the current sources should be matched in order not to give rise to inequalities between sources due to IR drop.

\subsubsection{Output resistance}

The finite output resistance of a current source results in variations in the output current that are dependent on the output voltage. This results in mainly second order distortion, which is suppressed by the differential nature of the architecture. However there are also higher order distortion components present due to finite, constant, output resistance of the current sources which are not suppressed by the differential architecture. The INL of a differential DAC with sources that have finite output resistance is given by [31]: 


$$
I N L=\frac{-g_{L} g_{o}^{2} k(2 k-N)(N-k)}{2\left(g_{L}^{2}+g_{L} g_{o} N+g_{o}^{2} k N-g_{o}^{2} k\right)\left(g_{L}+N g_{o}\right)}
$$

Here $g_{L}$ is the load conductance, $g_{o}$ is the output conductance of a unit cell, $k$ is the input code and $N$ equals $2^{\text {bits }}$, this is illustrated by figure 2-2. Figure 2-3 shows the calculated INL for a 10-bit DAC with $300 \mathrm{k} \Omega$ output resistance for a unit current source, into a $50 \Omega$ load.

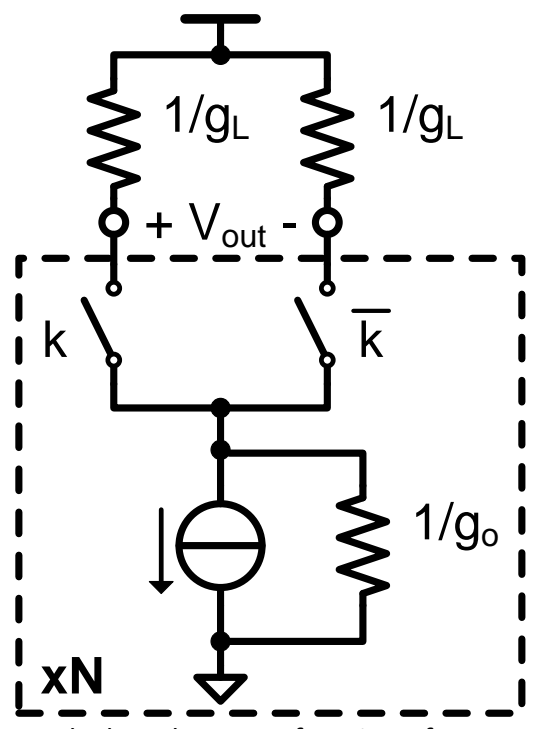

Figure 2-2: Definitions used to calculate the INL as function of current source output resistance

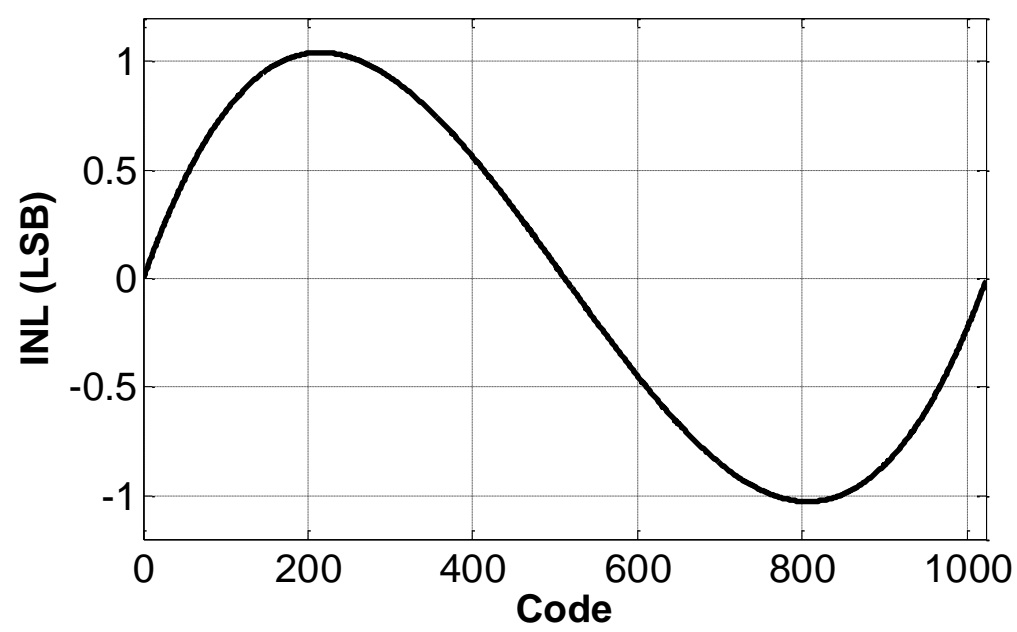

Figure 2-3: INL versus code of 10-bit DAC with $50 \Omega$ load and constant $300 \mathrm{k} \Omega$ unit output resistance 


\subsubsection{Output current variations}

Even when subjected to equal electrical conditions the current sources can have different output current due to a variety of causes. Mismatch is always a cause for variations between transistors. This is generally described by Pelgrom's Law [32](2-2), from which it follows that the variance of the output current of a current source is inversely proportional to the area of the current source transistor. So if the standard deviation needs to be decreased by a factor of two, the area needs to be increased by a factor four.

$$
\sigma=\frac{\mathrm{K}}{\sqrt{\mathrm{W} * \mathrm{~L}}}
$$

In this equation $\sigma$ is the standard deviation of the investigated parameter, $K$ is the standard deviation for a $1 \mu \mathrm{m}^{2}$ device, and $W$ and $L$ are the dimensions in $\mu \mathrm{m}$.

In addition to this mismatch, also gradients due to any non-uniformities in the chip fabrication play a role. Generally common-centroid layouts are used to limit their influence, although the exact influence of this gradient when a small current source matrix is used with today's large wavers is unknown.

Finally proximity effects also play an important role. Each current source should 'see' the same environment: if a current source at the edge of the matrix is close to another well, this will affect its characteristics. For this reason the current sources need to be surrounded by dummy devices, which have as goal to create an equal environment for all the actually used current sources.

\subsection{Dynamic mechanisms}

Many significant dynamic error mechanisms are present in CS DACs. One of the major dynamic error mechanisms is non-exact timing in the data switches. Timing errors can be variable, due to e.g. data-dependent clock loading, or they can be static, due to e.g. random mismatch or layout issues. For high-speed DACs with sample frequencies above $1 \mathrm{GHz}$ and moderate to high linearity requirements (higher than $50 \mathrm{~dB}$ ), timing errors are required to be in the sub-picosecond range, which is tough to achieve.

Other timing related errors are due to e.g. break-before-make behavior of switches that have periodically both switches in their off-state during switching, leaving the current source disabled and forcing some kind of recovery behavior after switching. Further 
significant timing related error mechanisms are due to differences in rise and fall times of the switches and effects such as clock feed through that all create spurs in the DAC's output signal.

In conventional CS DACs, the data switches switch only if the new code is different from the previous code: the amount of switching is hence code-dependent. Code-dependent switching introduces code-dependent load on the power supply, and induces disturbances to e.g. the bias lines. Both of these effects yield unwanted modulation of the output signal. Current-mode logic may be used to reduce the impact of this, but for complete suppression the switching fundamentally needs to be data-independent, which can for example be achieved with RZ-switching or quad-switching [33].

A last significant source of dynamic errors is the output capacitance of the current sources. While this capacitance usually is very linear, these capacitances are datadependently switched to either the positive or the negative output. Together with the load impedance they form a code-dependent RC filter, which results in spurs.

All these dynamic error mechanisms start at the switching time instance and last for a fraction of the sample period. The timing and switching related errors can have a large impact despite occurring only for a picosecond or even less.

\subsection{Recent Literature}

In the recent years new techniques have been developed to deal with dynamic errors that limit high-speed performance in CMOS DACs. Recently published DACs with high sample rates and >6-bit resolution are discussed in sections 2.3.1 through 2.3.5.

\subsubsection{Extra Cascodes with Bleeding Current Sources}

In $[34,35]$ a solution is proposed to eliminate the error due to the code-dependent capacitance seen at the output terminals of the DAC. This solution is shown in figure 2-4. 


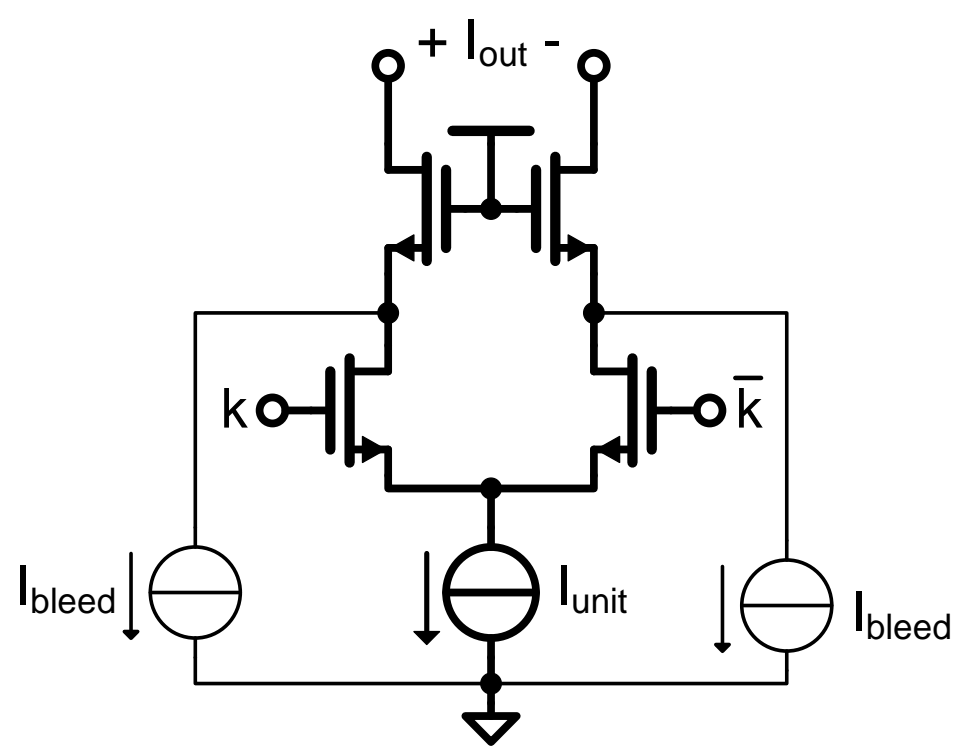

Figure 2-4: Extra cascodes and bleeding current sources to reduce influence of current source output capacitance

Cascode transistors are added at both the positive and the negative output of each DAC slice. In addition to this also at each output a small fixed current source is added. This current source is intended to keep the cascode transistor permanently in the same operating region, so that at the output node the code-dependent capacitance variations are greatly reduced. This does not completely eliminate the problem: the cascode capacitance itself will still be somewhat code-dependent, and it does not provide infinite isolation. This error reduction technique comes at the cost of a higher power consumption due the extra voltage headroom that is required and due to the current used by the bleeding current sources.

\subsubsection{Return-to-Zero Switching}

Code-dependencies in the switching behavior of DACs can be suppressed by implementing return-to-zero switching. This is generally done by adding extra switching for the zero part in the output $[30,36]$, although in recent years an alternative has been proposed: DRRZ (Digital Random Return-to-Zero) [37, 38, 39] or DMRZ/DEMDRZ (Dynamic-element-Matching and Digital Return-to-Zero) [22]. Both of these are essentially the same: the zero-phase of the output is not implemented by using separate switches, but instead by switching half of the current sources to the positive output, and the other half to the negative output. This in itself is not sufficient to 
suppress spurs. However there are many settings which result in equal positive and negative output currents which can implement the zero-phases, the number of possible combinations is given by $\left(\begin{array}{c}2^{\text {bits }} \\ 2^{\text {bits-1 }}\end{array}\right)$. For a 6 -bit section this equals $1.8 E 18$ combinations. By digitally randomizing the combination which is used at every zerophase, spurs are effectively transformed into noise and the advantages of RZ switching schemes are obtained without extra switches being required.

However at the same time return-to-zero switching also has significant downsides [40]. With the same output voltage swing they deliver less output power in the primary Nyquist zone, while the power of the image frequencies is larger, requiring steeper antialias filters. Additionally they are more sensitive to jitter, and the internal switching frequencies need to be higher compared to a NRZ DAC, limiting its usefulness for very high-speed DACs.

\subsubsection{Quad-Switching}

Quad-switching is an older technique, first shown in [41], and more recently in [15]. Instead of one set of switches per current source to redirect the current to the positive or to the negative output, it uses four switches as shown in figure 2-5, with inputs $k_{1}, k_{2}, \overline{k_{3}}$ and $\overline{k_{4}}$. Of these inputs only one is high at a time.

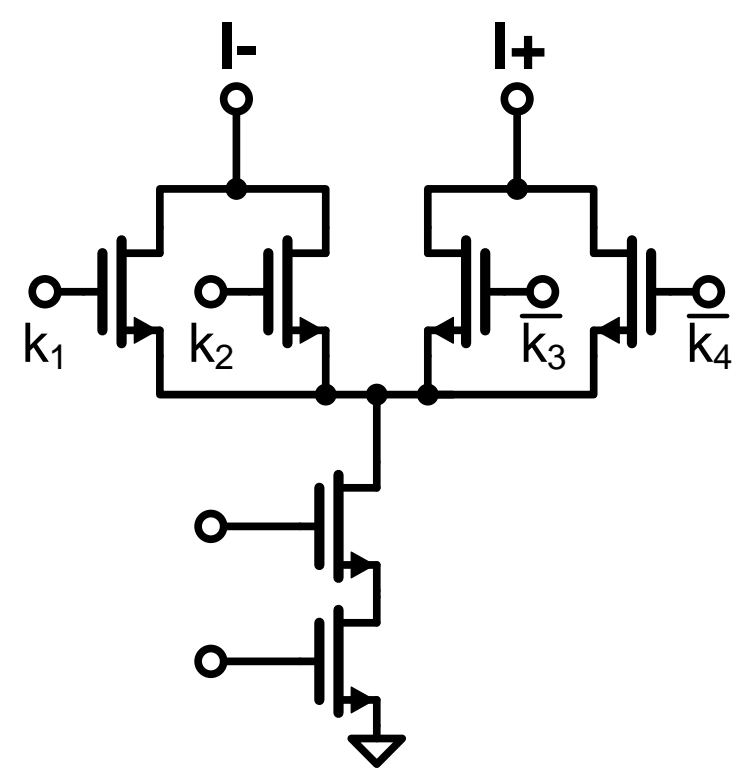

Figure 2-5: Quad-Switching Current Cell 
In a regular architecture the switch activity depends on whether the code changes or not. In a quad-switching architecture each clock cycle one switch turns off, and one switch turns on, removing the code-dependency from the switching activity. This is illustrated by figure 2-6 and further elaborated on in chapter 5 . The downside of this is that the average switching activity increases, and it costs a bit more area. Another potential problem is that the number of switches increase, and so does the potential for timing related problems. Part of these can be moved out of the signal band by running the quad-switching at twice the data rate [42], however doubling the switch frequency without actually increasing the sample rate is also not ideal for high-speed DACs. [43] presents this technique as dual return-to-zero, although normally dual return-to-zero consists of two RZ DACs in parallel, which is clearly not the case here.
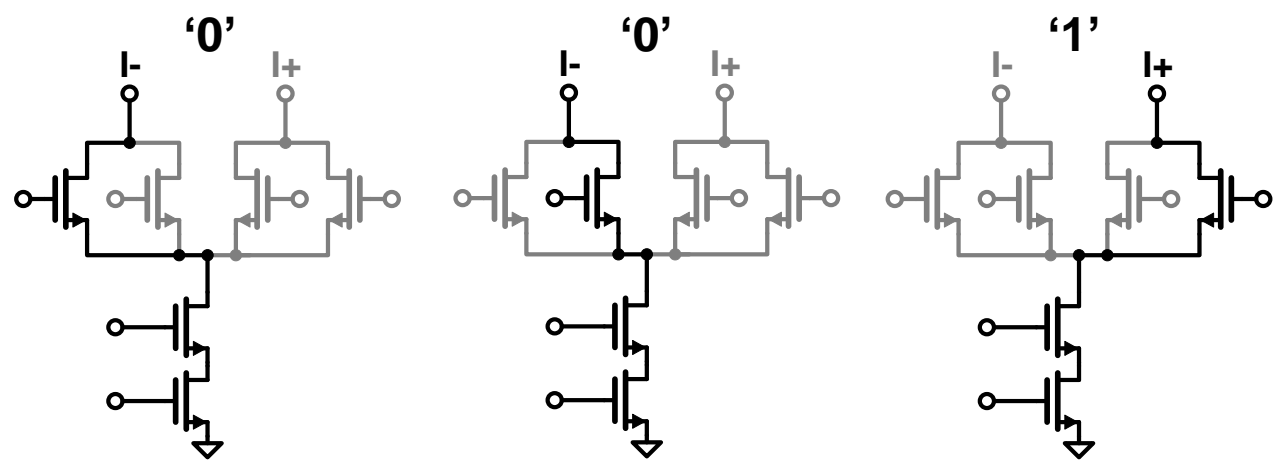

Figure 2-6: Quad-switching states for three consecutive clock cycles with outputs ' 0 '- ' 0 '-' 1 '. The grey transistors are turned off, while the black ones are enabled

\subsubsection{Interleaved DACs}

The interleaved CS DAC architecture that is the main topic of this thesis, has in recent years also been presented by other groups.

The work presented in [44] also uses an interleaved architecture to suppress switching induced spurs. They included digital pre-distortion to further lower some of the spurs, and made different design decisions compared to the work presented here; the system level design is similar, but the implementation of the sub-blocks are different. This results in very good IM3 performance, below $-74 \mathrm{dBc}$ up to $1 \mathrm{GHz}$, at the cost of a high power consumption $(2.3 \mathrm{~W})$ and core area $\left(5.2 \mathrm{~mm}^{2}\right)$. This is one-two orders more than what is used by our designs in chapter 4 and 5 .

Another type of interleaved DAC, which is discussed in [45, 46, 47], also consists of several sub-DACs in parallel, however in contrast to the work presented in this thesis 30 
and the work of [44], no multiplexer is used to combine them and instead the output currents of all sub-DACs are directly summed. While this does still double the sample rate, it does not provide the other benefits that an interleaved architecture can give. When the connected sub-DACs are RZ DACs, this is also presented as dual return-tozero [40].

In $[48,49,50,51]$ also interleaved architectures are shown, however these have only reported idealized simulation results, which cannot be compared to measured results.

\subsubsection{Other publications}

Many other publications are available that try to reduce distortion components of highspeed DACs. For example reduction of differences between different binary scaled sources was achieved by adding replica circuits in [52]. In [53,54] high operating speed with low power consumption is achieved using a binary structure which consists of parallel unit cells, instead of combining them as one large cell, concluding that at least for a limited number of bits good performance can be achieved while using a binary decoding. At similar speeds [55] achieves improved performance by using a custom CML latch to improve performance, and where the DAC is optimized for low area, which also limits the size of layout parasitics. Additionally it also employs replicas for reducing differences between different bits, however these are operated at reduced current to lower power consumption.

While full sigma-delta implementations are not fast enough yet for the frequencies considered here, the hybrid implementation shown in [56], which uses both a Nyquist rate part and a sigma-delta part, achieves a signal bandwidth of $500 \mathrm{MHz}$ with good linearity, although that is including digital predistortion.

By randomizing which unit cells are used at any time to create a binary scaled source, [57] can decrease the mismatch induced distortion components. In [58] an active calibration is used to reorder the switching sequence such that the INL is optimized: a smaller-than-nominal current cell will be followed by a larger-than-nominal one. This sorting algorithm is further improved on in [59], which uses a 3D calibration technique. This method is not only capable of reducing distortion at low, but also at high frequencies by pairing current cells with opposite (compensating) behavior. This seems to work well for IM3 performance, boosting it by $10 \mathrm{~dB}$ over Nyquist, but appears to have little influence on the SFDR. 
By adding extra switches that can invert the signal, [60] is effectively integrating a current-mode mixer. This mixer has a fixed frequency equal to the sampling rate and a variable duty cycle. This adds the option to invert the output current during a part of each sample period, allowing the DAC to make effective use of multiple Nyquist zones. The main advantage compared to simply sampling faster should be found in the relative low power consumption. 


\section{Chapter 3 \\ The Interleaved Structure}

This chapter is based on [61], starting from section I-B up to section II, and section IV in [61]. Compared to [61] the differences between different operating region for the multiplexer transistors is described more in-depth.

The dynamic errors in CS DACs are present at the switching time and during a short period after the switching time instances. During the remainder of a sampling period, the effect of these dynamic errors can be sufficiently small. Consequently, the linearity of a CS DAC can be improved if we make sure the DAC is not connected to the output during the time that the dynamic errors are significant; this is for example done in [30, 36] in the form of an RZ output signal. However as mentioned in section 2.2, RZ results in much larger transients and increases demands on analog post-filtering while at the same time the delivered output power is decreased. This can be improved by using two sub-DACs (sDACs) that operate alternatingly by using opposite clock phases: then each SDAC can be connected to a dummy output during the switching moment thereby placing the timing and settling related errors on only the dummy output. Once settled, the SDAC's output can be routed to the actual output, and meanwhile the other SDAC can switch to and settle to its new code. The corresponding interleaved architecture for this is shown in figure 3-1. In this figure SDAC-A and SDAC-B are alternatingly switched to the actual output and to a dummy output by the multiplexer. Note that while this interleaved approach doubles the required area and power compares to the RZ variant, it also doubles the sampling rate without requiring higher switching frequencies and outputs a regular, non-return-to-zero, waveform. 
Several other interleaving architectures are known from literature; a brief discussion is given below. Placing multiple sDACs in parallel and shorting their outputs is sometimes classified as interleaving [62]. However while this is easy to implement and while it does double the sampling rate, it does not solve issues such as timing mismatch and codedependent settling speed. Since it sums the currents it does not output the converted digital input word, but the sum of the last two, modifying the frequency response. This last issue can be solved by implementing RZ switching in each sDAC cell [63] which makes sure that only the current code is converted to the output, and at the same time it adds some of the advantages of an RZ DAC. However it does not remove all of the timing and settling issues associated with conventional RZ DACs.

In this paper, the focus is on two-times-interleaved CS DAC architectures with a central multiplexer to combine the outputs. Higher interleaving counts can be used, but twotimes interleaving will already suppress all timing errors sufficiently by giving enough time for settling of nodes.

Using an interleaved architecture as low-power, area-efficient solution might seem counter-intuitive at first; placing two sDACs in parallel doubles both area and power consumption, and additionally also an analog multiplexer is required to toggle between the two. However since both sDACs only run at half the overall DACs speed with significantly reduced demands on dynamic errors for each SDAC due to the interleaving setup, each individual sDAC can actually be small and low-power, while maintaining a good overall interleaved DAC performance.

Interleaved DACs employing an analog multiplexer have been reported before. The work in [64] contains the first reference to this method of removing switching transients from the output of a DAC; using an opamp with a built-in multiplexer to switch between two sDACs. In [49] a method to limit the impact of gain mismatch between sDACs is presented and illustrated only using simulations on an idealized circuit. The interleaved DACs in [65], discussed in chapter 4 of this thesis, and [66], discussed in chapter 5 , use triode switches without quad-switching to obtain $58 \mathrm{~dB}$ SFDR across Nyquist at $1.7 \mathrm{GS} / \mathrm{s}$. In [44] saturation switches are used; large bleeder currents are added to improve their linearity. While the design in [44] achieves superior SFDR, $69 \mathrm{~dB}$, this is across less than a quarter Nyquist at $4.6 \mathrm{GS} / \mathrm{s}$ and at a cost of more than one order higher power consumption and two orders in area compared to the work in this paper. 

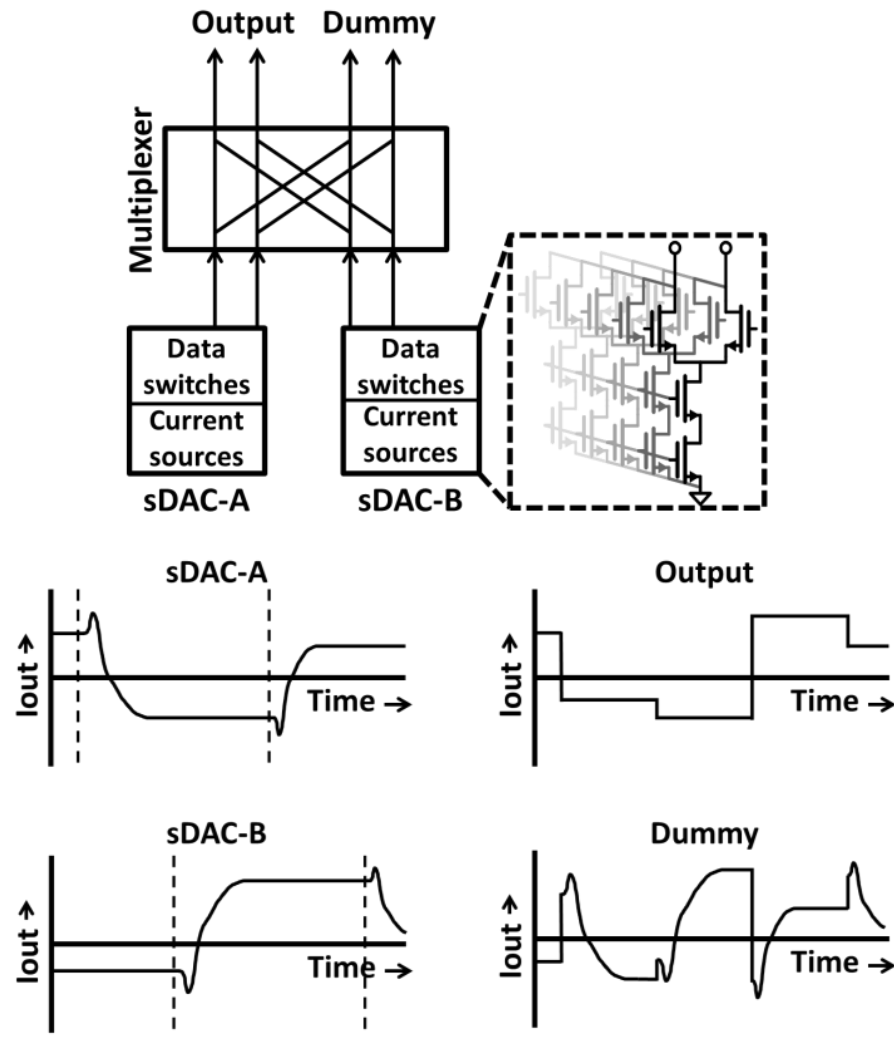

Figure 3-1: Interleaved architecture

\subsection{Interleaving errors mechanisms}

While the interleaved architecture suppresses most of the regular dynamic CS-DAC errors, it also introduces new errors that may limit performance if not dealt with correctly. The sDACs need to be matched well, both in their code-output signal transfer and in the time that they are connected to the output. Note that the first property is due to the matching of the two sDACs while the second property is determined by the analog multiplexer that toggles between the two sDACs.

In a regular CS-DAC all data switches route the current of their associated current source to either output; hence the switches switch a static code-independent current. In the interleaved architecture, the switches in each individual SDAC also switch static code-independent currents, but the analog multiplexer that toggles between both 
sDACs switches the full, code-dependent, output current. This results in additional challenges to obtain good spectral performance compared to conventional CS DACs.

The following sections discuss the most important issues in interleaved DACs.

\subsubsection{Static matching of the sDACs}

To simplify the analysis of the overall DAC performance limitation due to static SDAC mismatch, the output signal of the second SDAC is written as that of the first SDAC with a code-dependent mismatch term: $V_{\text {out }, s D A C 2}(\operatorname{code})=V_{\text {out }, S D A C 1}(\operatorname{code})+\epsilon(\operatorname{code})$. Each of the SDACs in the interleaved DAC runs at half the sampling frequency, with their corresponding image frequencies. For perfectly matched sDACs, $\epsilon(\operatorname{code}) \equiv 0$ and the image frequencies due to interleaving cancel each other. In that case, the harmonics and images created by the interleaved DAC due to e.g. any non-linearity in $V_{\text {out,sDAC1 }}$ (code) are exactly the same as that of a non-interleaved DAC having the same $V_{\text {out }, D A C}(\operatorname{code})$. Any mismatch $\epsilon$ (code) between the sDACs however limits the cancellation of the image frequencies, while these images fall in-band for the complete interleaved DAC when assuming full Nyquist operation.

In a conventional non-interleaved DAC, a DC offset has no effect on its performance. In an interleaved DAC, a DC offset $\epsilon(\operatorname{code})=\Delta$ between the two sDACs results in a square wave shaped output signal running at half the sampling frequency, creating a spur at half-FS and at DC. An illustration of this error mechanism is shown figure 3-2; the output signals of both sDACs are shown in the top graph for a sinusoidal output, where the output signal of sDAC-B equals the sum of the output signal that sDAC-A would give for the same code and of a fixed offset. The lower graph in figure 3-2 shows only this error component, scaled up by a factor 5 for illustration purposes. These resulting spurs reside outside the Nyquist band. 

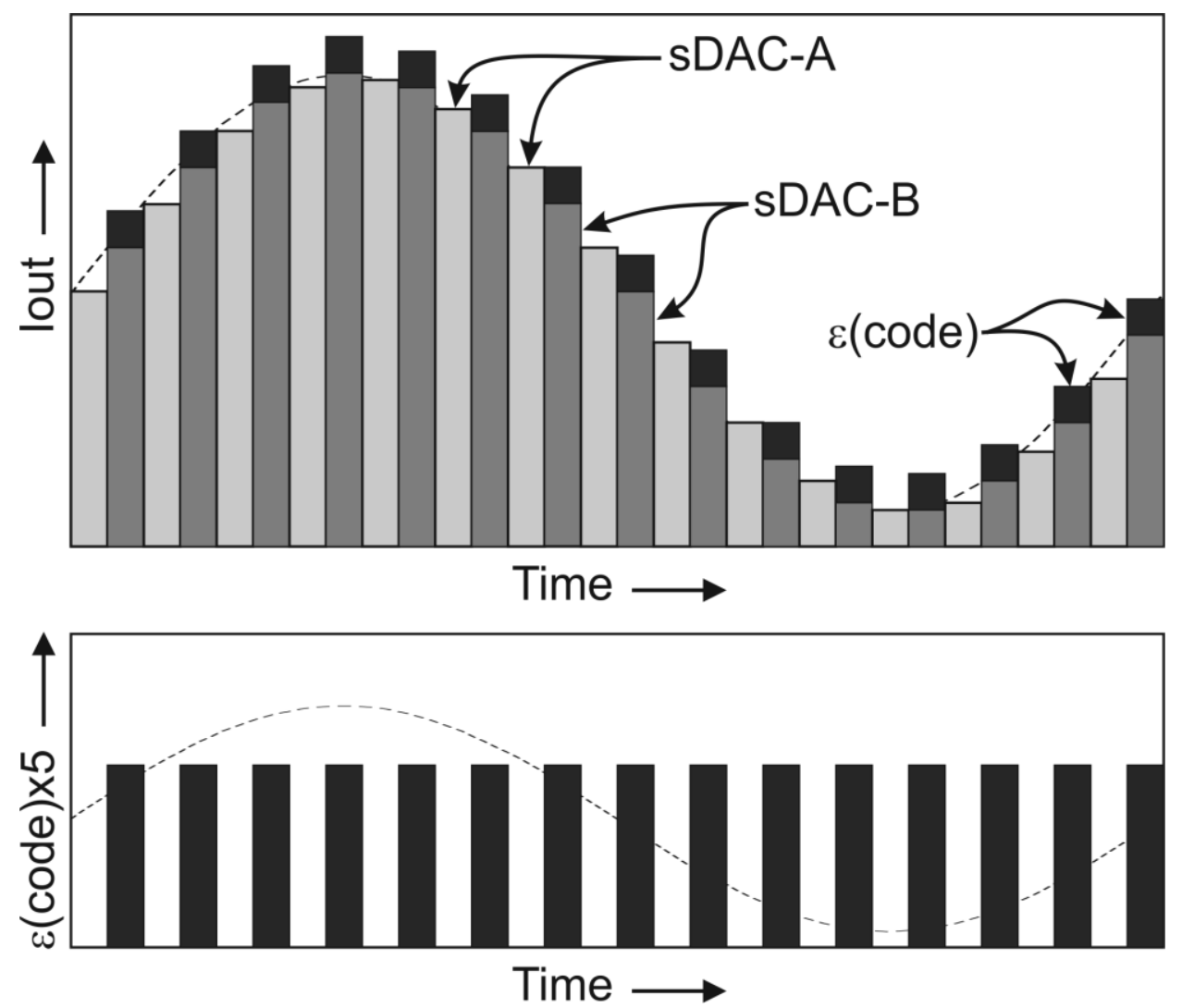

Figure 3-2: Output of an interleaved DAC with an offset error between the sDACs

Similarly, a gain error has no effect on the output spectrum in a conventional DAC. In the interleaved DAC, a gain error $\epsilon($ code $)=\varepsilon_{\text {gain }} * V_{\text {out }, \text { SDAC1 }}($ code $)$ generates an RZ output signal proportional to the output signal, at half the sample frequency of the overall system. Figure 3-3 shows an illustration of this error mechanism: the output signals of both sDACs are shown in the top graph for a sinusoidal output. The output of SDAC-B is subdivided into the response that SDAC-A would have given for the same code and into the error contribution $\epsilon$ (code). The lower graph in figure 3-3 shows only this error component, scaled up by a factor 5 , which is proportional to a $50 \%$ duty cycle RZversion of the DAC's output signal. 

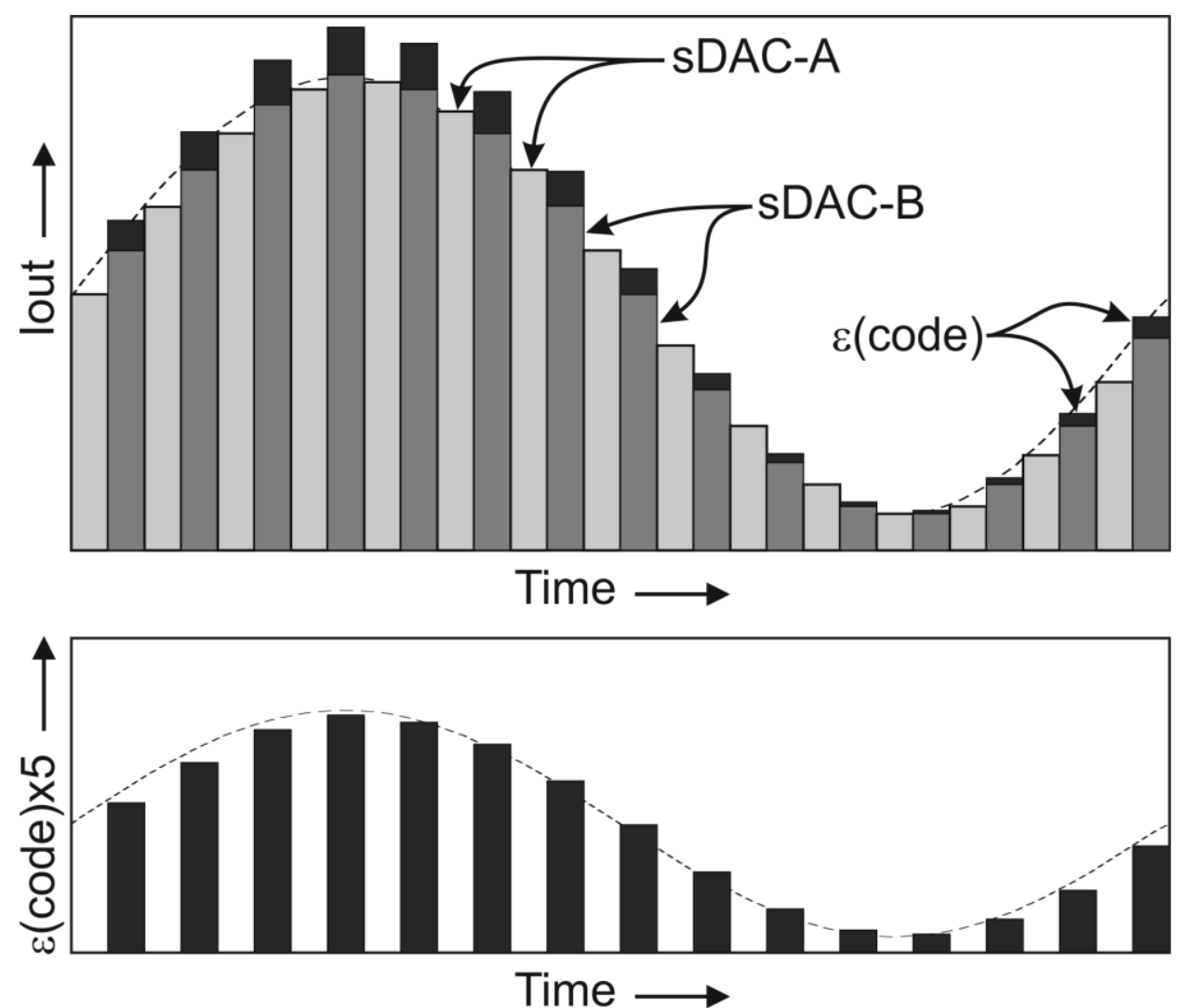

Figure 3-3: Output of an interleaved DAC with a gain error between the SDACs

Since $\epsilon$ (code) has half the sampling frequency of the complete DAC, it generates signals at exactly the output frequency, adding to the main signal, and generating spurs mirrored around half Nyquist:

$$
f_{\text {spur }}=\frac{f_{\text {sample }}}{2}-f_{\text {signal }}
$$

Ignoring the frequency roll-off due to zero-order hold behavior, the SFDR of the resulting return-to-zero signal is given by:

$$
S F D R=20 \log _{10}\left(\frac{1}{\epsilon_{\text {gain }}}\right)+6 \mathrm{~dB}
$$

Where the $6 \mathrm{~dB}$ is due to the $50 \%$ duty cycle of the $\mathrm{RZ}$ signal. When the zero-order hold behavior is taken into account the fundamental has a $\operatorname{sinc}(x)$-shaped frequency response. The image is a return-to-zero signal at half the sampling frequency, yielding an equal sinc-shaped frequency response with $6 \mathrm{~dB}$ attenuation. Noting that the image 
frequency is mirrored around half Nyquist compared to the fundamental, the overall attenuation of the image compared to the fundamental is given by:

$$
\text { Image }_{\text {attenuation }}=20 \log _{10}\left(\frac{\operatorname{sinc}\left(\frac{f_{\text {signal }}}{f_{\text {sample }}}\right)}{\operatorname{sinc}\left(\frac{\frac{f_{\text {sample }}}{2}-f_{\text {signal }}}{f_{\text {sample }}}\right)}\right)
$$

At (near) DC signal frequency, the relative attenuation of the image according to (3-3) is $4 \mathrm{~dB}$, while at (near) Nyquist signal frequency this relative image attenuation is $-4 \mathrm{~dB}$. This yields:

$$
\begin{gathered}
S F D R_{D C}=20 \log _{10}\left(\frac{1}{\epsilon_{\text {gain }}}\right)+10 \mathrm{~dB} \\
S F D R_{\text {Nyquist }}=20 \log _{10}\left(\frac{1}{\epsilon_{\text {gain }}}\right)+2 \mathrm{~dB}
\end{gathered}
$$

Higher order mismatch between the sDACs, where $\epsilon(\operatorname{code})=\varepsilon_{n} * V_{\text {out,sDAC1 }}^{n}($ code $)$, gives harmonics of the fundamental in the output current and due to the RZ behavior these are also folded around half-Nyquist. The effect of this higher order mismatch also result in spurs in a similar way as in CS DACs; so the required matching to suppress the spurs in interleaved DACs is also similar to the matching required for a regular CS DAC. It does not matter if this higher order mismatch is common for the SDACs or opposite to each other for the size of the resulting spur.

\subsubsection{Dynamic matching of the multiplexer}

Mismatch in the multiplexer and its driver result in a non-50\% duty cycle for the connection of either SDAC to the overall output, which in turn yields spurs in the DAC output signal. With a non-50\% duty cycle, one SDAC (e.g. the signal due to one code) is still at the output, while the next one should already have been placed on the output. Since this happens only at every second transition, an error is created which occurs at only half the sample rate of the complete DAC. This spur is located at the same frequency as an amplitude error (3-1).

Assuming a sinusoidal fundamental signal with unit amplitude and ignoring the sampled nature of the DACs output signal, the size of the error $\epsilon(\operatorname{code})$ can easily be estimated. 
Under these assumptions, the error due to non-50\% duty cycle switching between the two sDACs is a pulse train, at half the sample frequency. The amplitude of these pulses is the difference between two consecutive samples, which can be approximated by the derivative of the fundamental signal multiplied by the sample period, i.e. divided by its sample frequency. In this approximation, the sinc roll-off is ignored which effectively yields an overestimation of the error.

$$
\epsilon_{\text {amp-pulse }}=\frac{2 \pi f_{\text {signal }}}{f_{\text {sample }}} \cos \left(2 \pi f_{\text {signal }} t\right)
$$

The duty cycle of the error pulse train is determined by the timing error, denoted as $\Delta t$, divided by two sample times, since it only occurs once every two samples:

$$
\epsilon_{\text {duty-pulse }}=\frac{1}{2} \Delta t f_{\text {sample }}
$$

An illustration of this error mechanism and of the resulting error signal is given in figure 3-4. The output signals of both SDACs are shown in the top graph for a sinusoidal output, for a $60 \%$ duty cycle for SDAC-A; the output of SDAC-A is subdivided into the response for $50 \%$ duty cycles and into an error contribution $\epsilon$ (code). The lower graph in figure 3-3 shows only this error component, scaled up by a factor 3 .

If the sDACs' duty cycle is almost $50 \%$, the error pulse train has a small duty cycle $\epsilon_{\text {duty-pulse }}$, and the roll-off of its output spectrum is negligible for the first Nyquist zone. The magnitude of the spurs in the signal band is simply the product of the error amplitude in (3-6) and the duty cycle in (3-7). The SFDR due to only the spurs originating from a non-50\% duty cycle for the sDACs is the ratio between the fundamental signal and this error signal. The fundamental does suffer from a sinc roll-off as does the error signal amplitude, which was ignored in (3-6). Since these two sinc-roll-offs have an equal dependency on the fundamental frequency, they cancel each other when calculating the SFDR. This yields the following expression for SFDR due to duty cycle errors:

$$
S F D R=20 \log _{10}\left(\frac{1}{\pi f_{\text {signal }} \Delta t}\right)
$$


For the presented $11 \mathrm{GS} / \mathrm{s}$ full-Nyquist DAC this sets the maximum allowed timing error at less than $200 \mathrm{fs}$ to be able to reach $50 \mathrm{~dB}$ SNDR, which is a stringent requirement.

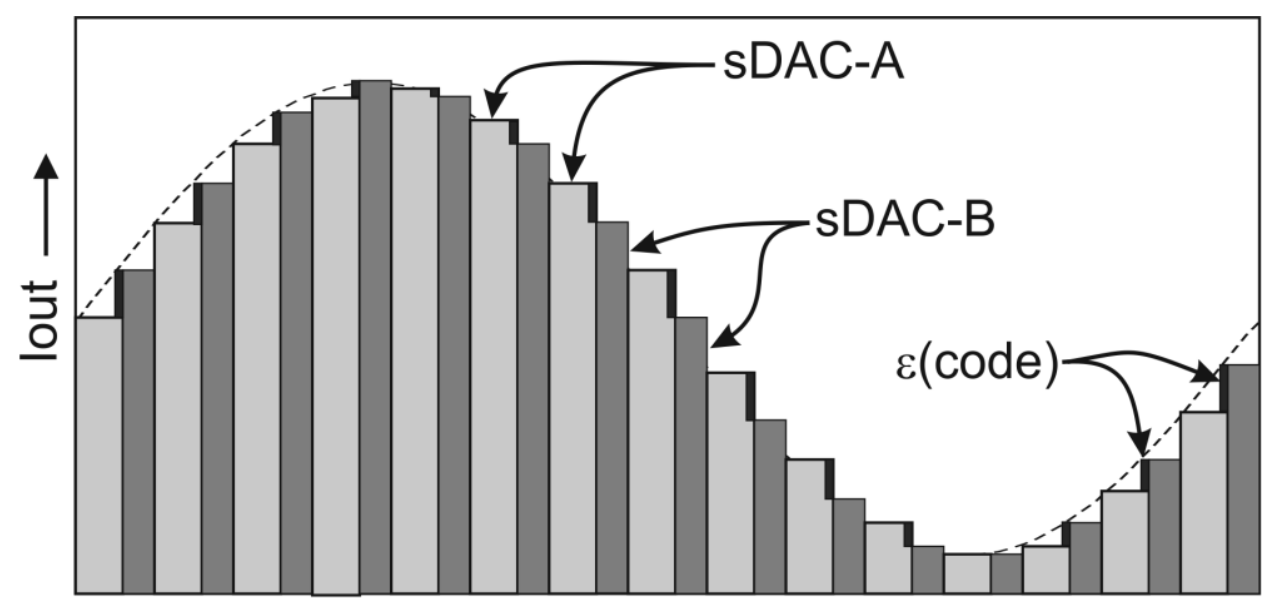

Time

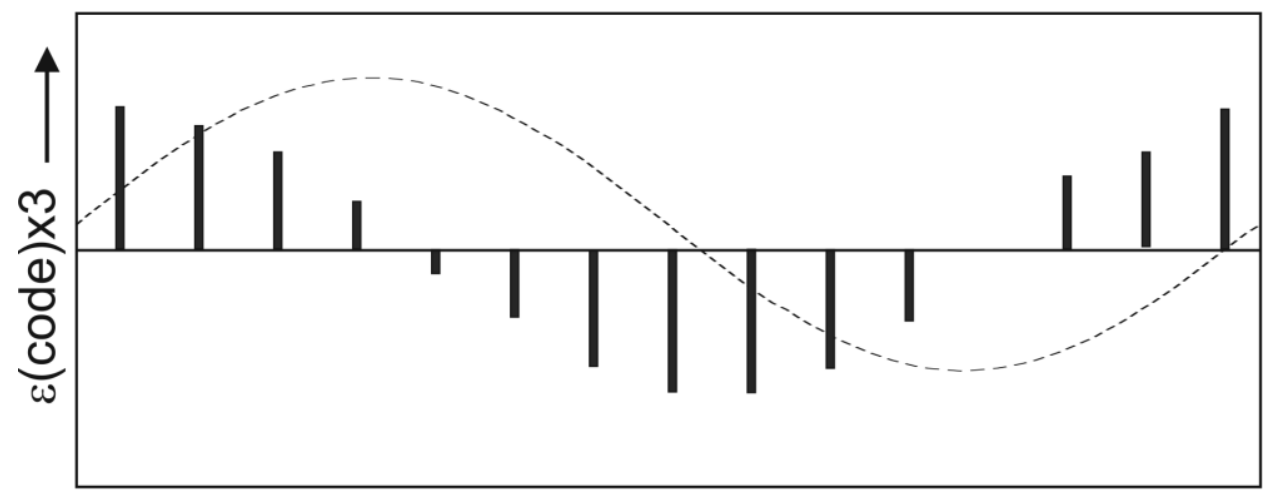

Time

Figure 3-4: Output of an interleaved DAC with a duty cycle error

\subsubsection{Multiplexer transistor nonlinearities}

Switches in regular CS DACs only have to switch a fixed (code-independent) current which is one of the reasons they are suitable for high-speed operation. In contrast, the multiplexer transistors in an interleaved DAC switch the output signal of the full sDACs, these signals are by definition code-dependent and which hence may yield codedependent spurs. 
This code-dependency results in for example a varying load impedance seen by the sDACs, which results in code-dependent settling speed, creating spurs in the output spectrum. Ideally in an interleaved DAC structure as presented in this thesis this is irrelevant, since all signals in an SDAC should be fully settled before that SDAC is connected to the output by the multiplexer. However for example charge injection from the fairly large multiplexer switches also has to settle, similar to transients caused by bondwire inductance and these are not suppressed in the interleaved structure. The multiplexer switches can be implemented with saturation or triode mosfets.

Saturation switches have as advantages that they provide reverse isolation and, if sufficiently wide, provide a low-ohmic load impedance for the sDACs. This low-ohmic loading of each SDAC decreases the signal swing at the source node of the multiplexers, improving SDAC linearity, and allowing for a larger output swing without the multiplexer transistors leaving their operating area. The downside is that the multiplexer's transistors behavior is highly dependent on drain current. At low drain current levels their transconductance is low, providing a relatively high-ohmic load to the sDACs. Extra bias current or bleeders can reduce this problem, but relatively high levels of extra bias current would be required to get sufficiently low code-independent loading effects. Another issue with multiplexer transistors in saturation is that the DC voltage level of the output voltage of the DAC must be sufficiently high to ensure that these transistors stay in saturation which would increases power consumption and decreases the voltage headroom at the output of the DAC.

Triode switches operate largely independent of their drain current: their on-resistance is a relatively weak function of drain current compared to saturation switches. Since a triode switch in its on-state appears as a resistance in series with the load resistance, the sDACs see a relative constant load. Furthermore, triode switches are bi-directional: both positive and negative currents (the latter due to e.g. charge dump) are properly routed to the dummy output. A last advantage of using triode switches over conventional saturation switches is that the drain-source voltage in triode is small which maximizes the voltage headroom. A drawback of using triode switches is that the source voltage of the multiplexer switches experience almost the full swing of the DAC output signal; this increases demands on the output impedance of the sDACs and requires operation of the triode switches in deep triode. To illustrate the differences between saturation and triode switches, figure 3-5 shows the simulated impedance at the source node of a transistor in different bias conditions, assuming the square law 
transistor model. Operating the transistor in saturation, the source impedance of the transistor approaches infinity at lower currents which is not acceptable to route SDAC currents. Using a $50 \%$ full scale bleeding current, the source impedance is much more current independent. Operating the same transistor in triode, the source impedance is higher than that for operation in saturation with $50 \%$ full scale bleeding but is also much more current-independent, while it requires a lower voltage headroom and can properly handle bi-directional currents. The low power interleaved DACs in this thesis hence implements deep triode switches.

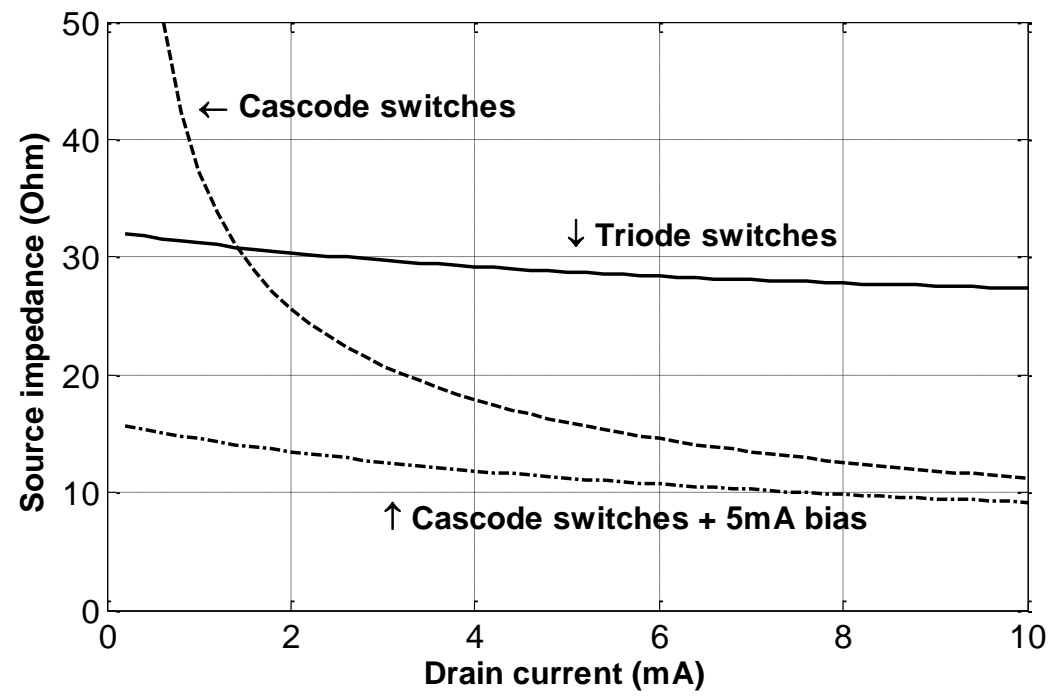

Figure 3-5: Impedance seen by the sDACs, for different multiplexer operating regions

\subsection{Measuring duty cycle}

In our design, the multiplexer driver achieves good timing accuracy because in the (symmetrical) layout it is positioned in the symmetry plane while it is optimized for maximum passive matching. However, passive matching alone is not sufficient to reach the required timing accuracy required for a decent SFDR, see e.g. (3-8). Duty cycle calibration is implemented to solve timing issues; this requires both a means to accurately measure timing and circuitry that can tune the timing in the sub-picosecond range.

Direct measurement of sub-picosecond timing requires high-end measurement equipment. However, interleaved DACs only the timing error must be known and must be tuned to zero. This tuning error can easily be estimated using (3-1): a calibration 
signal at half the sampling frequency generates a timing spur at DC. This DC spur can be measured and tuned to zero using e.g. an auxiliary ADC with low linearity and low signal bandwidth. Generating this calibration signal with an interleaved DAC is straightforward: both sDACs are placed at a constant, opposite, code. Assuming the amplitudes of the SDACs are equal, any error in the duty cycle will result in a non-zero DC differential output voltage level.

In practice there will be offsets in the measurement, for example due to mismatch in the load resistors or in the ADC itself. By swapping the SDAC codes these offsets can be cancelled, via the same principle as a chopper amplifier is based on. This also allows for doing the calibration single-endedly instead of differential. Large variations in supply voltage or temperature can require new calibrations to keep the duty cycle error sufficiently small. However for small changes this is not necessary since the majority of the variation in delay due to these variations will be common mode for the positive and negative switch signals.

During calibration the signal is at Nyquist while the calibration attempts to remove all spurs at DC. If also amplitude mismatch is present, this creates a spur at DC as well. The algorithm tries to cancel this spur by introducing a duty cycle error that creates a spur with equal size but opposite phase, cancelling out the amplitude spur.

For signals near DC, a duty cycle error has little effect while the amplitude error is attenuated by $10 \mathrm{~dB}$, see (3-4). With just an amplitude error present this would decrease to $2 \mathrm{~dB}$ at Nyquist, see (3-5). However with increasing signal frequency, the size of the duty cycle spur (with opposite phase compared to the amplitude spur) increases and increasingly cancels the amplitude spur. Then the combined spur magnitude decreases with increasing frequency; ideally at Nyquist the two spurs cancel out each other perfectly. From this it follows that in a timing calibrated interleaved DAC the minimum attenuation of the amplitude error spur is $10 \mathrm{~dB}$. This means that if a $50 \mathrm{~dB}$ SFDR is required, the amplitude difference between the two SDACs is allowed to be up to $1 \%$, or slightly over 5 LSB for a 9-bit DAC. The SFDR as function of co-existing amplitude and timing errors is discussed more extensively in Appendix $A$, where also equations are derived for the (partial) cancellation of the amplitude-mismatch induced spur by the timing related spur. 


\section{Chapter 4 \\ Medium Speed Demonstrator: 12-bits at 1.7GS/s}

In order to verify effectiveness of using the interleaved architecture presented in the previous chapter to design a fast and accurate DAC, a 12-bit, DAC was designed in $65 \mathrm{~nm}$ CMOS. The goal was to design a DAC competitive with state-of-the-art in the low GS/s range, which would - depending on the exact sampling frequency - require an SFDR of $60 \mathrm{~dB}$ or better. For this linearity 12-bit is more than sufficient, so performance is not limited by the number of bits, while adding more bits increases complexity for no further gain. The realized DAC was set to operate at $1.7 \mathrm{GS} / \mathrm{s}$, to balance linearity and sample rate in the final results.

In this chapter the designed chip and its performance is introduced, followed by a conclusion regarding its effectiveness. This chapter is based on [65], compared to [65] more explanations regarding the design are included while also additional measurements results are shown.

\subsection{Architecture}

In the designed chip, each sDAC essentially is a complete current DAC, including separate bias sources and decoder logic. The sDACs have a 6-6 segmentation, where the MSB sources are implemented with thermometer code and the LSB sources use binary code. Their output current goes to the SDAC multiplexer, which alternatingly connects one SDAC to the output, and the other one to an identical dummy output. The resistive load consists of four $50 \mathrm{Ohm}$ on-chip resistors; two single-ended loads for each of the two differential outputs, in parallel to four 50 Ohm off-chip resistors. 


\subsubsection{Static matching}

The current sources of the two sDACs have equal process spread, but a (largely) uncorrelated mismatch; therefore their INL curves are also (largely) uncorrelated. When interleaving, the difference in INL between the 2 sDACs causes an (interleaving) output spur located at a frequency equal to the fundamental tone mirrored around half Nyquist. The only way to eliminate this interleaving spur is to get similar (preferably identical) INL curves for both SDACs, e.g. using calibration. For calibration purposes small (digitally controlled) calibration current sources are included in parallel to all MSB thermometer current sources and the two largest LSB binary scaled sources. The smaller LSB current sources will have a sufficiently low contribution to the overall INL/DNL of the system to not limit performance.

A conventional way to calibrate would be using a (more than) 12-bit linear ADC to calibrate the INL of both SDACs to near zero. Since this method allows for direct, accurate, calibration of the complete transfer curve, it prevents large INL errors due to cumulative residual errors after calibration of current sources. However this conventional way does require a relatively complex $A D C$ because it needs to meet the DAC's high linearity requirements over the full output signal range.

The calibration for our interleaved DAC is different and requires only a low-linearity $A D C$. Calibration happens at a relative low speed, so the ADC also does not need to be fast. A faster $A D C$ does reduce the time calibrations required. During the calibration, first one of the SDACs is calibrated towards a sufficiently low DNL-INL and then the second SDAC is calibrated to get the same INL behavior.

This calibration is done in two steps. Firstly to calibrate the first SDAC, each thermometer MSB source (having a nominal weight of 64LSB) is calibrated to be equal to all LSB sources combined (a nominal weight of 63LSB) + one extra LSB source. Now the calibrating $A D C$ only has to compare the value of the MSB source with a reference value and hence the ADC only needs to be monotonic. Note that this method actually calibrates the DNL, but due to cumulative errors the INL can still have a significant error. Subsequently, the transfer of the second SDAC is calibrated to match the transfer of the first SDAC by calibrating the second SDACs MSB sources.

The result is that the INL of the second SDAC follows the INL of the first one: so the second SDAC has an INL error, but there is only a minor residual difference between the 
INLs of the SDACs. During this calibration phase, we only have to measure the difference between two values: again there are very low demands on the linearity of the calibrating ADC.

\subsubsection{Dynamic matching}

In interleaved DACs, the time intervals in which each SDAC is connected to the DAC output must be equal, otherwise spurs mirrored around half Nyquist occur, as discussed in section 3.1.2.

From (3-8) it follows that for a full Nyquist signal at $1.7 \mathrm{GS} / \mathrm{s}$ and an SFDR $>70 \mathrm{~dB}$, the static timing error must be smaller than $110 \mathrm{fs}$. These levels of timing accuracy practically cannot be obtained by matching only: also here calibration is required.

In our design we use a quasi-DC measurement to calibrate the timing. In it, first one of the sDACs is set to its maximum output value while the other one is set to its minimum value. The resulting average output voltage is dependent on the signals amplitude (previously calibrated, see section 4.1.1) and the duty cycle of the generated square wave. The calibration can be repeated with the first SDAC at its minimum and the second at its maximum to further decrease mismatch effects.

\subsection{Circuit implementation details}

An advantage of the interleaved architecture is that the SDAC design is relative simple and straightforward. The basic current steering architecture depicted in figure 1-12, a cascoded current source with two data switches on top of them, is used for the sDACs.

Since the MSB and two largest LSB current sources are calibrated, they do not need to have high intrinsic matching. The unit current cells are dimensioned at $0.45 \mu \mathrm{m} / 330 \mu \mathrm{m}$, with cascodes sized $0.2 \mu \mathrm{m} / 0.15 \mu \mathrm{m}$. They are grouped to together form the different current sources required in the SDAC. The MSB current sources, which consist of 60 unit sources, each have 5 smaller current sources in parallel for calibration purposes, allowing them to be calibrated between 60 and 67.75 unit sources with steps of $1 / 4^{\text {th }}$ unit source. Each of these sources have an SRAM memory cell which can be programmed via the external control bus of the chip to turn them on/off. The largest LSB source has 4 calibration current sources, and 30 fixed unit sources, while the second largest LSB source has 3 calibration sources and 15 fixed unit sources. The remaining LSB's only have fixed sources: respectively 8, 4, 2 and 1 . 


\subsubsection{Multiplexer and driver design}

The timing calibration uses the timing generation circuit in figure 4-1 to adjust the edges of the clock signal until the duty cycle is sufficiently close to $50 \%$. The circuit first divides the input frequency by 2; a digitally controlled 2x6-bit capacitor bank can slightly delay both clock signals. Finally, the two SR-latches convert the time-delayed signals to two anti-phase square waves with a duty cycle that depends on the delay settings. The delay-range that can be created this way is \pm 3 . $3 p s$, with $50 \mathrm{fs}$ - $55 \mathrm{fs}$ steps.

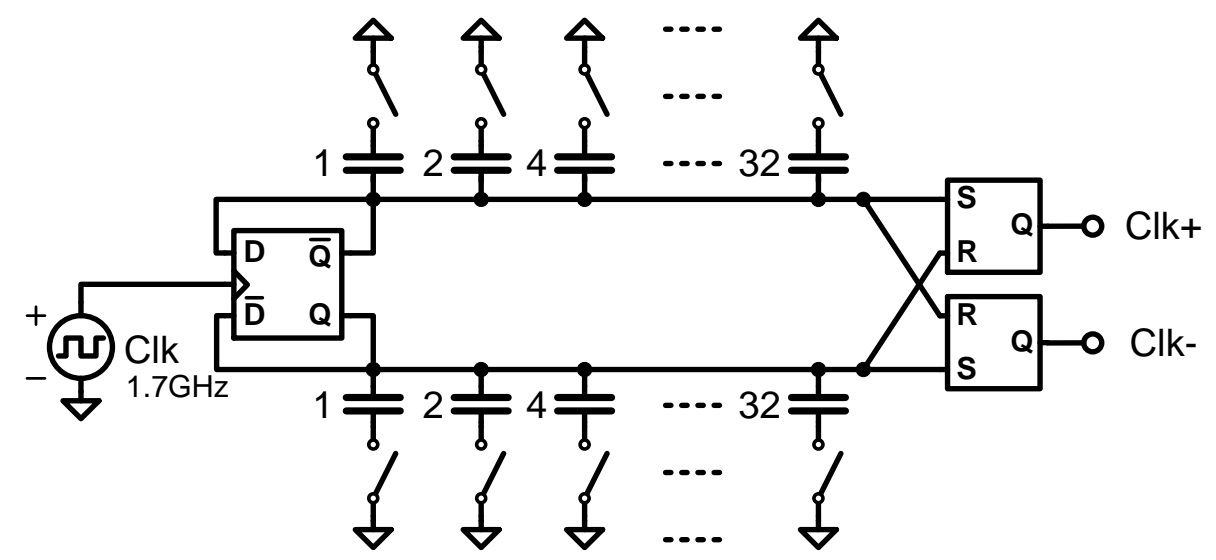

Figure 4-1: Timing generation + timing calibration circuitry

The outputs of this circuit drives both the analog multiplexer that combines the two sDAC outputs, and the sDACs themselves. In the previous chapter the rationale for using the multiplexer switches in triode was shown. In the demonstrated design in this chapter, an integrated (straight forward) capacitive level shift circuit is used to create gate drive voltages between $0.9 \mathrm{~V}$ and $2 \mathrm{~V}$ to operate the multiplexer transistors in triode. This circuit is shown in figure 4-2.

\subsubsection{Bias design}

For the interleaving architecture to function properly, each SDAC should be completely settled before its output is connected to the output. This includes the bias and (analog) power lines. The power lines could stabilize in time with sufficiently short bondwires, however due to uncertainty about their inductance in the used QFN package in our demonstrator design at design time, it was instead decided to use a large amount of decoupling capacitors to deal with disturbances. The majority of these capacitors are poly capacitors. While these have a relative high series resistance, the interleaved 
architecture gives them some extra time to make sure the voltage at the relevant locations is settled in time.

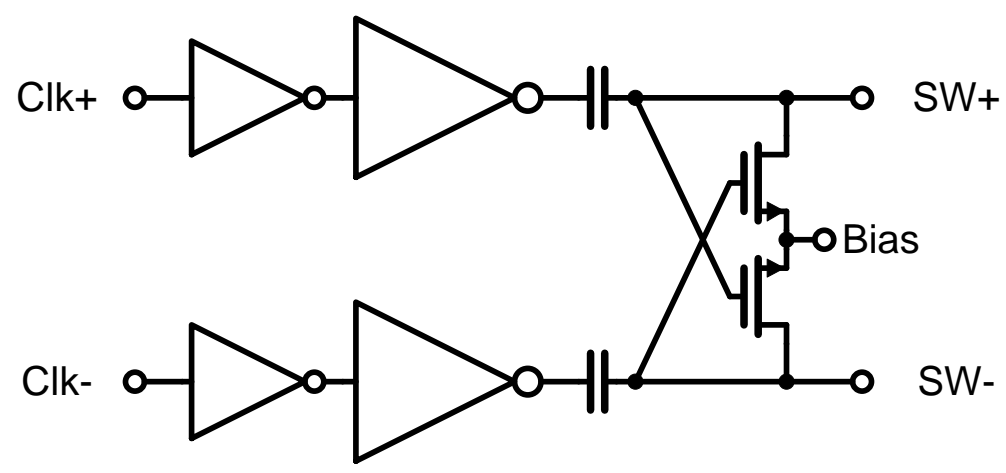

Figure 4-2: Multiplexer level shifter

Sufficiently reducing disturbance on the bias lines by means of adding large amounts of decoupling capacitors would be problematic from a layout perspective. The solution used here is minimizing the capacitance on the bias lines while also minimizing the real part of the output impedance of the bias generator: i.e. a low ohmic reference voltage with a small parasitic capacitance. By doing so, the recovery time of disturbances on the bias lines is minimized, yielding well settled bias voltages when the sDAC is connected to the output.

As shown in figure 3-1 the current sources consist of two transistors, one operates as current source, the other one as cascode to boost the output impedance. The biasing for the current source transistors and the cascode transistors are generated by the circuit from figure 4-3. The current source transistor is biased using a diode connected mosfet with length equal to that of the current source transistor. A current is forced through the mosfet $M_{3}$, generating a reference voltage. This setup can have a low output impedance, if a sufficient amount of current is used.

A stable and fast recovering reference is also important for the cascode transistor's gate voltage. The cascode reference-voltage is just as the current source reference-voltage implemented using a diode connected mosfet to allow for fast settling. However, this is complicated since the cascode bias level needs to be a higher voltage than the current source bias level. Three methods are used to increase the gate-source voltage of this diode-connected transistor. 


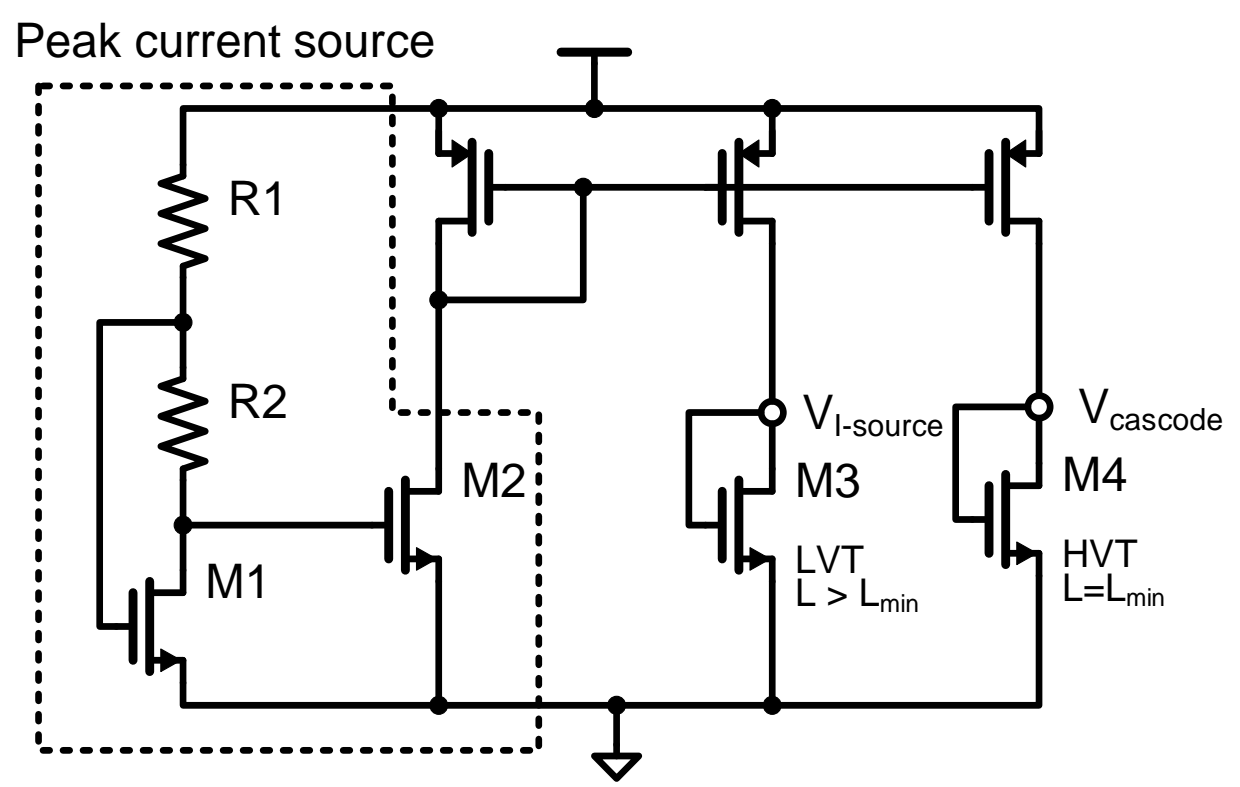

Figure 4-3: Biasing circuit for the current sources

Firstly, using the reverse short channel effects of our transistors, the threshold voltage is maximized using a minimum length devices. Secondly while all other mosfets are lowthreshold devices, this mosfet is a high-threshold device, increasing the generated voltage further. These high-threshold devices will not match exactly with the regular devices over PVT (Process, Voltage and Temperature) variation. However since the exact voltage of the cascode transistors is not critical, this is acceptable in this design. Finally the width is decreased to generate a suitable voltage. While this has a negative influence on the output impedance of this diode-connected reference transistor, the effect is small enough to keep the overall output resistance is sufficiently low.

For good performance it is essential the current delivered by the current sources in the DAC core are stable. A possible source of disturbance would be from the supply lines, even though the bias circuits have a separate supply, coupling between supply domains could be an issue. To suppress this a peak current source has been implemented. This current source uses $R_{1}$ to generate a reference current. If $M_{1}$ has a transconductance equal to $1 / R_{2}$, variations in the current generated by $R_{1}$, for example due to supply variations, do not propagate to the drain voltage of $M_{1}$. This drain voltage is then used to create a stable reference current by means of $M_{2}$. The PMOS current mirror distributes this current to the diode connected mosfets. 


\subsubsection{Digital circuitry}

On the chip an integrated digital sine generator is included. This was designed by STM engineers. The sine generator is capable of generating both single-tone and two-tone test signals of varying frequencies and amplitudes. Also optionally the signal can be dithered.

Since the $6 \mathrm{MSBs}$ in the DAC core are thermometer coded, a thermometer decoder is required to control them. Even though in the layout there is no actual matrix floorplan used, a row/column based thermometer decoder is implemented because of its simplicity. This decoder consists of two 3-bit thermometer decoders, one for the rows and one for the columns. The used 3-bit thermometer decoders are drawn in figure 4-4. Each of the 7 thermometer outputs, $t_{0}$ till $t_{6}$ is created from the three binary inputs $b_{0}$, $b_{1}$ and $b_{3}$ using logic gates.
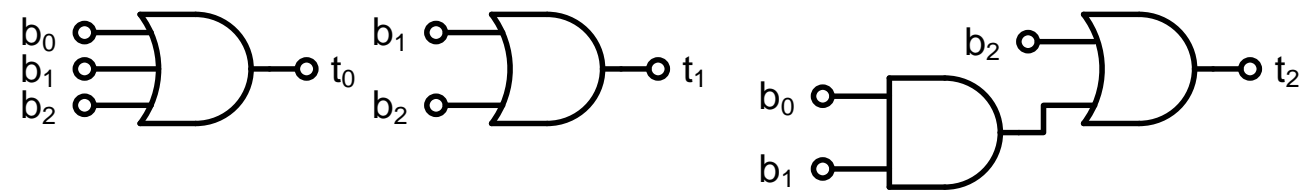

$b_{2}$

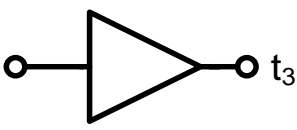

$b_{0}$
$b_{2}$
$b_{1}$
$b_{2}$

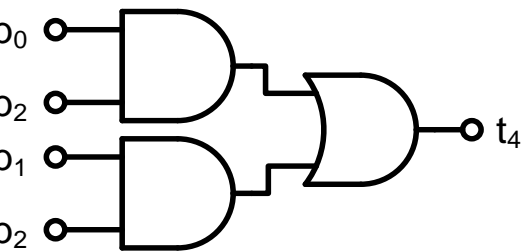

$b_{1}$

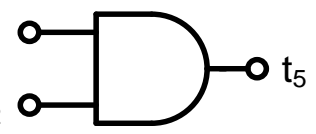

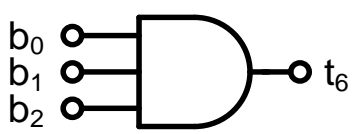

Figure 4-4: 3-bit thermometer decoder

The two 3-bit thermometer decoders are combined to create a single 6-bit thermometer decoder, this is depicted in figure 4-5. In this figure the circuit is drawn as a matrix for simplicity, however in the actual layout they are placed in a line. Each cell in the matrix uses the state of its corresponding column bit, and its row bit in addition to the next row bit, to calculate its state: A cell has a 'high' output when either its column input and its row input are both 'high', or when the next row is 'high'. A flipflop is used afterwards to reclock the signal. 
The total thermometer decoder has 63 outputs, which in a square matrix means 8 inputs on each side. The first three binary bits are used for the column decoder. The $8^{\text {th }}$ column input is always fixed at zero, the cells in this column are only enabled when the next row is activated. The other three inputs are used for the row decoder. Here the first row (at the bottom in the picture) is always 'high', the $9^{\text {th }}$ row is always 'low'. The last cell in the matrix, top-right, is always 'low'. This brings the total number of thermometer outputs at the expected 63.

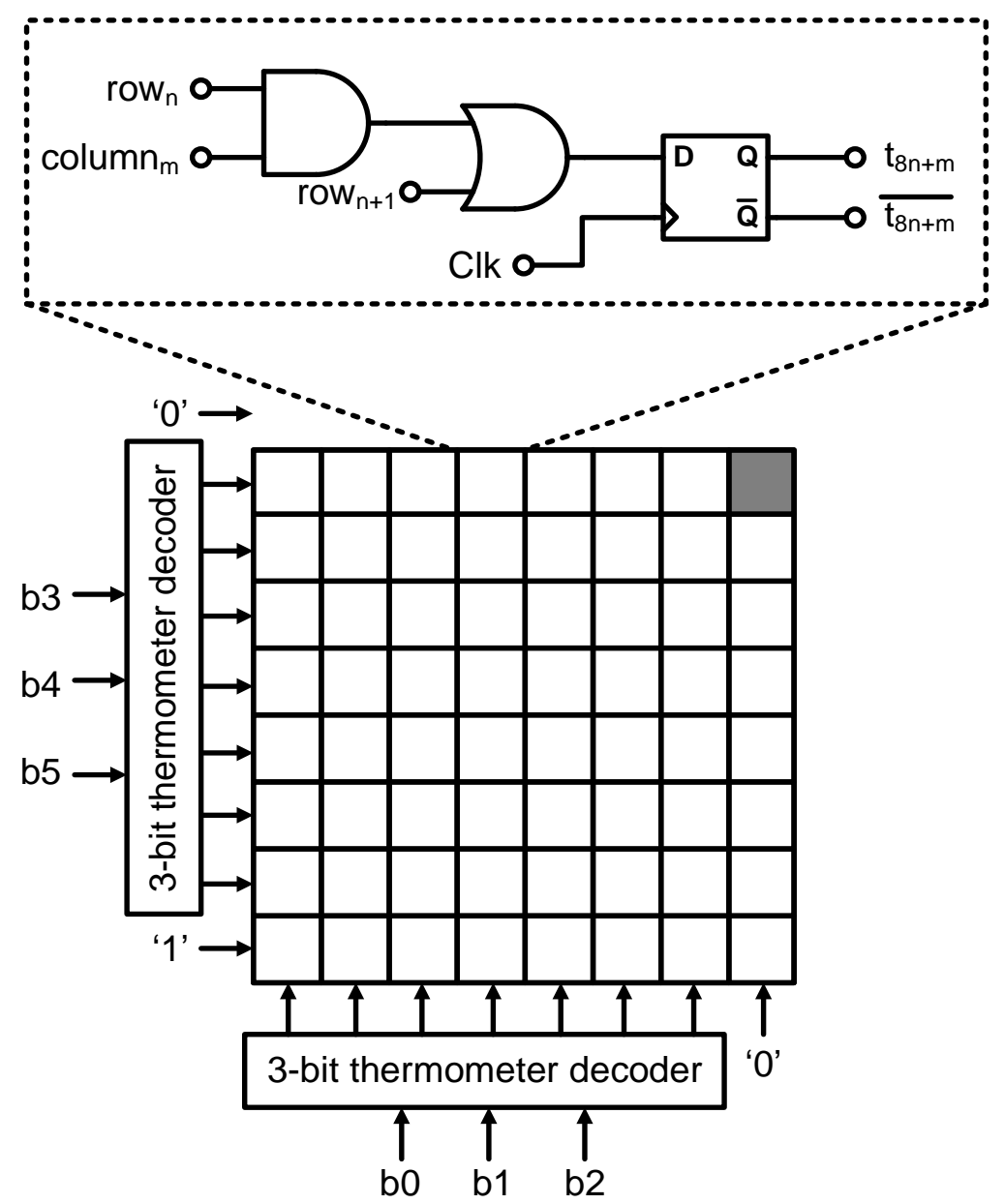

Figure 4-5: 6-bit thermometer decoder created using two 3-bit thermometer decoders 


\subsection{Measurement results}

The DAC was produced in a standard $65 \mathrm{~nm}$ CMOS technology from ST Microelectronics. Figure 4-6 shows the die micrograph. From it the symmetrical layout can be observed, which is required to prevent interleaving spurs. An earlier version did not have this symmetrical layout, which limited its performance. The resulting problems from this earlier version are discussed in Appendix C. The active area of the demonstrator is $0.4 \mathrm{~mm}^{2}$ and the chip is packaged in a standard QFN40 package. The IC consumes $70 \mathrm{~mW}$ from its single $1.2 \mathrm{~V}$ supply, excluding sine generator power consumption. Parallel to the internal $50 \Omega$ resistors, the DAC is externally loaded with $50 \Omega$ resistors, without the customary RF chokes which are generally required for sufficient voltage headroom. Both the internal and external resistors are connected to the $1.2 \mathrm{~V}$ supply. The interleaved DAC delivers $500 \mathrm{mVpp}$-diff full scale swing to the outputs.

\subsubsection{Static matching}

Figure 4-7 shows the measured static INL of the two SDACs before and after calibration. Calibration is performed using an Agilent 33401A multimeter which performs the role of ADC, with the calibration code running on Matlab, and setting the calibration SRAM cells using the serial bus of the chip.

Before calibration the two SDACs have different gains, resulting in a large difference in INL between the two SDACs. After calibration the INL is significantly improved, although the INL is still substantial because of both our rather noisy off-chip multimeter and because we actually only calibrated the DNL; cumulative errors in the calibrated DNL add up. Most importantly, the differential INL is minimized: the second SDAC clearly follows the INL curve of the first sDAC curve, despite the noisy ADC. Before calibration the best-fit full scale gain error is over 60LSB, after calibration this is less than 0.4LSB. 


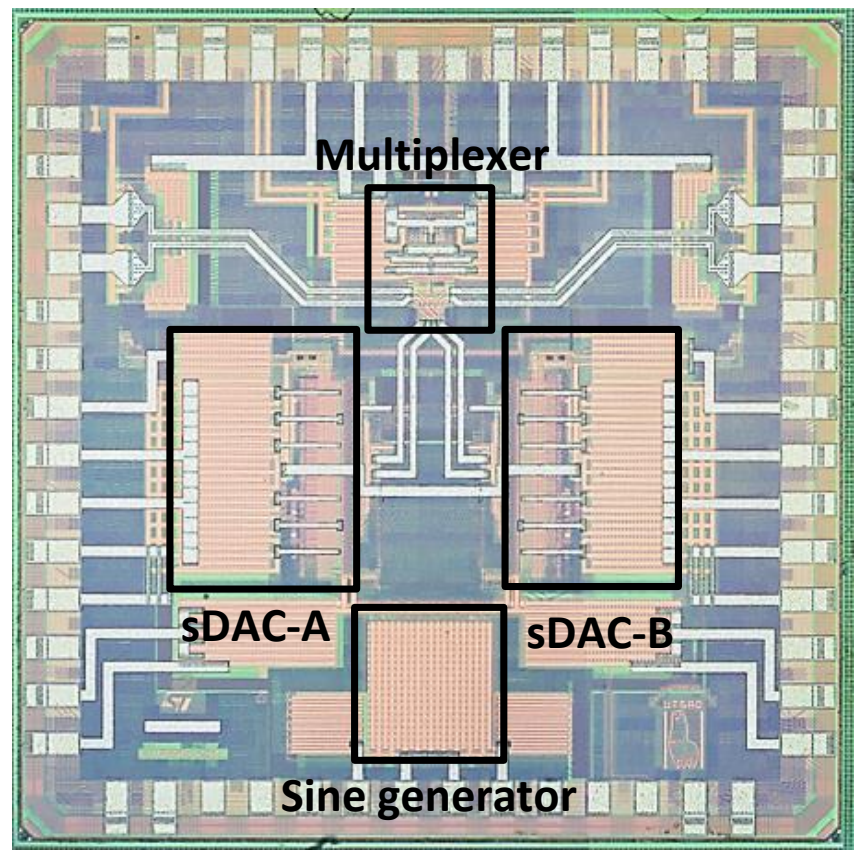

Figure 4-6: Die photograph, the complete chip size is $1.6 \mathrm{~mm} \times 1.6 \mathrm{~mm}$

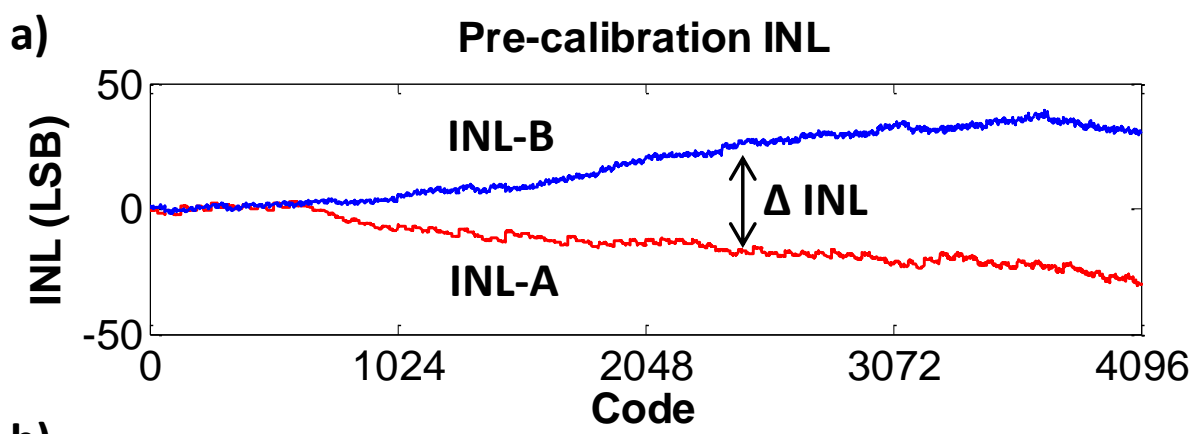

b)

Post-calibration INL

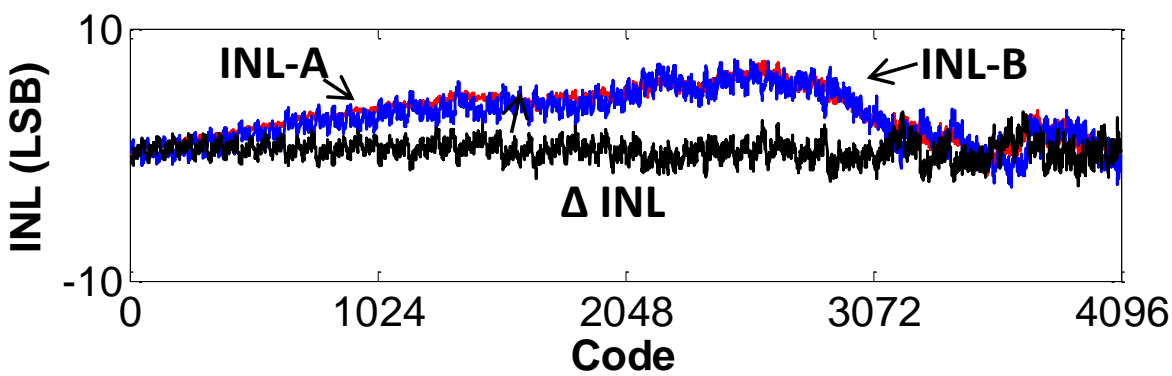

Figure 4-7: a) Static INL before and b) static INL after calibration 


\subsubsection{Dynamic matching}

The uncalibrated timing error was measured across 10 samples. This was done by the same DC measurement method that was used to calibrate the timing error out, described in section 3.2. The standard deviation of the calculated timing error of the measured samples equals $0.66 \mathrm{ps}$, with an average timing error of $0.29 \mathrm{ps}$. Figure $4-8$ shows the timing error measured for different digital calibration codes of the timing for an unselected sample. For both halves of the differential switch signal, the rising edge can be delayed in 63 digital steps, which gives a differential range of -63 to 63 . The total calibration range for the current design is 3.3ps. While this does not span the entire 3sigma range, from the measured values it indicates it should be sufficient for nearly $98 \%$ of the samples.

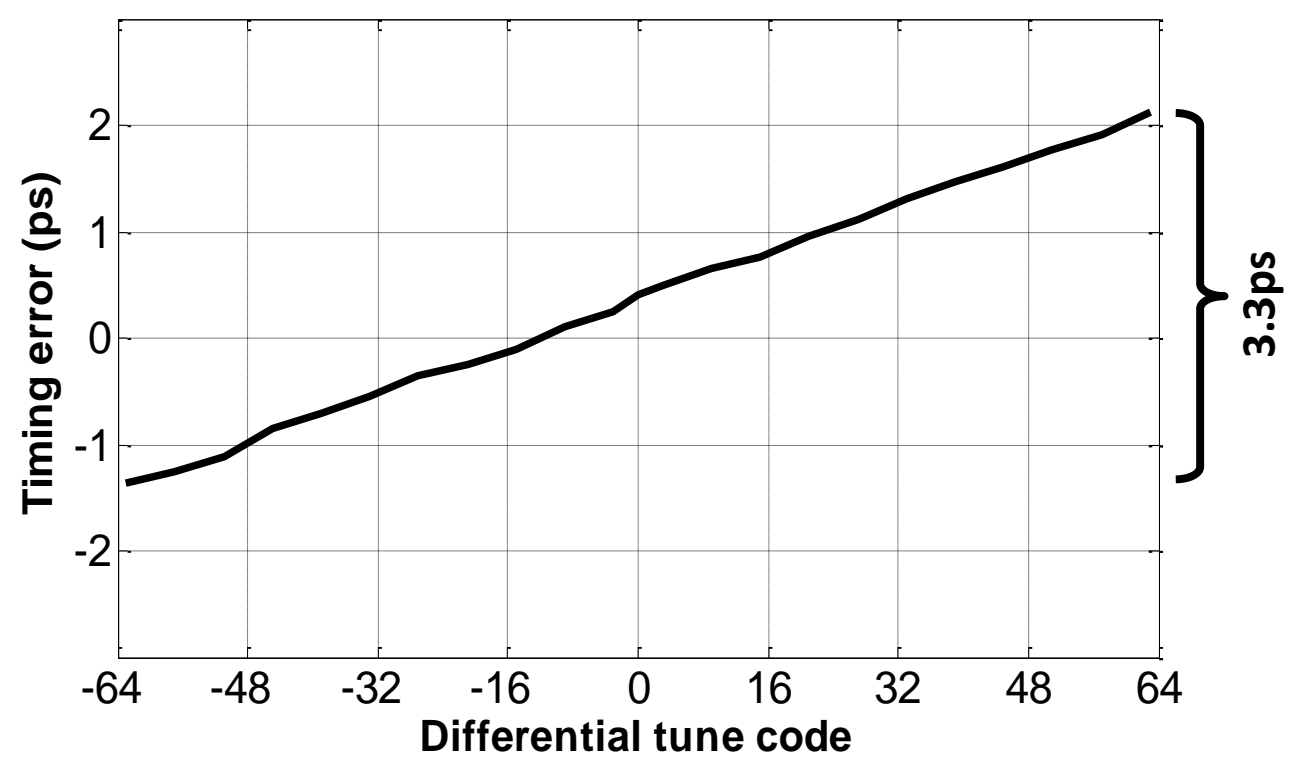

Figure 4-8: Measured timing error versus calibration code

\subsubsection{Spectrum}

The spectral performance of the DAC is shown in figure 4-9. The median SFDR over Nyquist is $60 \mathrm{~dB}$, with a minimum of $58 \mathrm{~dB}$. The IM3 is above $62 \mathrm{~dB}$ across Nyquist, with a median value of $73 \mathrm{~dB}$. Additionally it is clear that the interleaving spur does not limit performance; HD2 and HD3 spurs (which have different frequency dependencies) alternatingly limit the SFDR. At Nyquist the interleaving spur is at $-65 \mathrm{~dB}$; using (3-8) it follows that the timing error was calibrated to around $210 \mathrm{fs}$. 


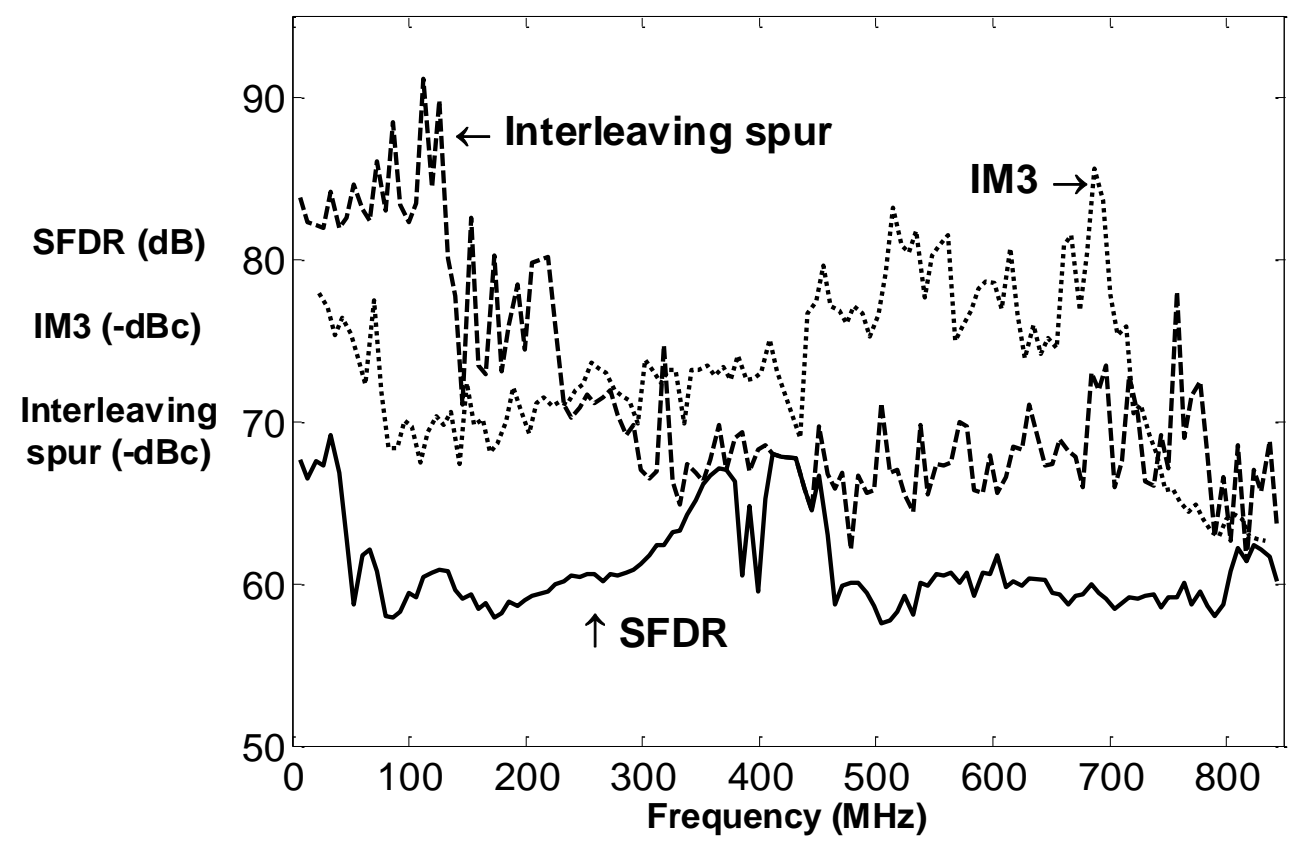

Figure 4-9: Spectral performance at 1.7GS/s

Figure 4-10 shows the spectrum for an output frequency of $604 \mathrm{MHz}$ at a sample rate of $1.7 \mathrm{GS} / \mathrm{s}$. The figure shows that for this frequency the HD3 is dominant, and that performance is not limited by the interleaving spur. At other frequencies the HD2 can be dominant, the interleaving spur does not typically limit performance.

In order to demonstrate the performance improvement achieved by interleaving, the performance of an individual SDAC was measured without interleaving using two-tone tests. Since the single SDAC runs at half the sample rate of the complete system, the SFDR of the two cases are hard to compare due to aliasing spurs in the output spectrum of the single sDAC. The IM3 obtained from two-tone tests does not have this problem, and can also be easily compared when the single SDAC is operating in its second Nyquist zone. In figure 4-11 the IM3 of the single SDAC is plotted against the frequency and compared to the IM3 of the complete system where interleaving is enabled. Above $425 \mathrm{MHz}$ the single sDAC enters its second Nyquist zone, where its performance drops rapidly,, but also for lower frequencies it performs far worse than the complete interleaved DAC system. 


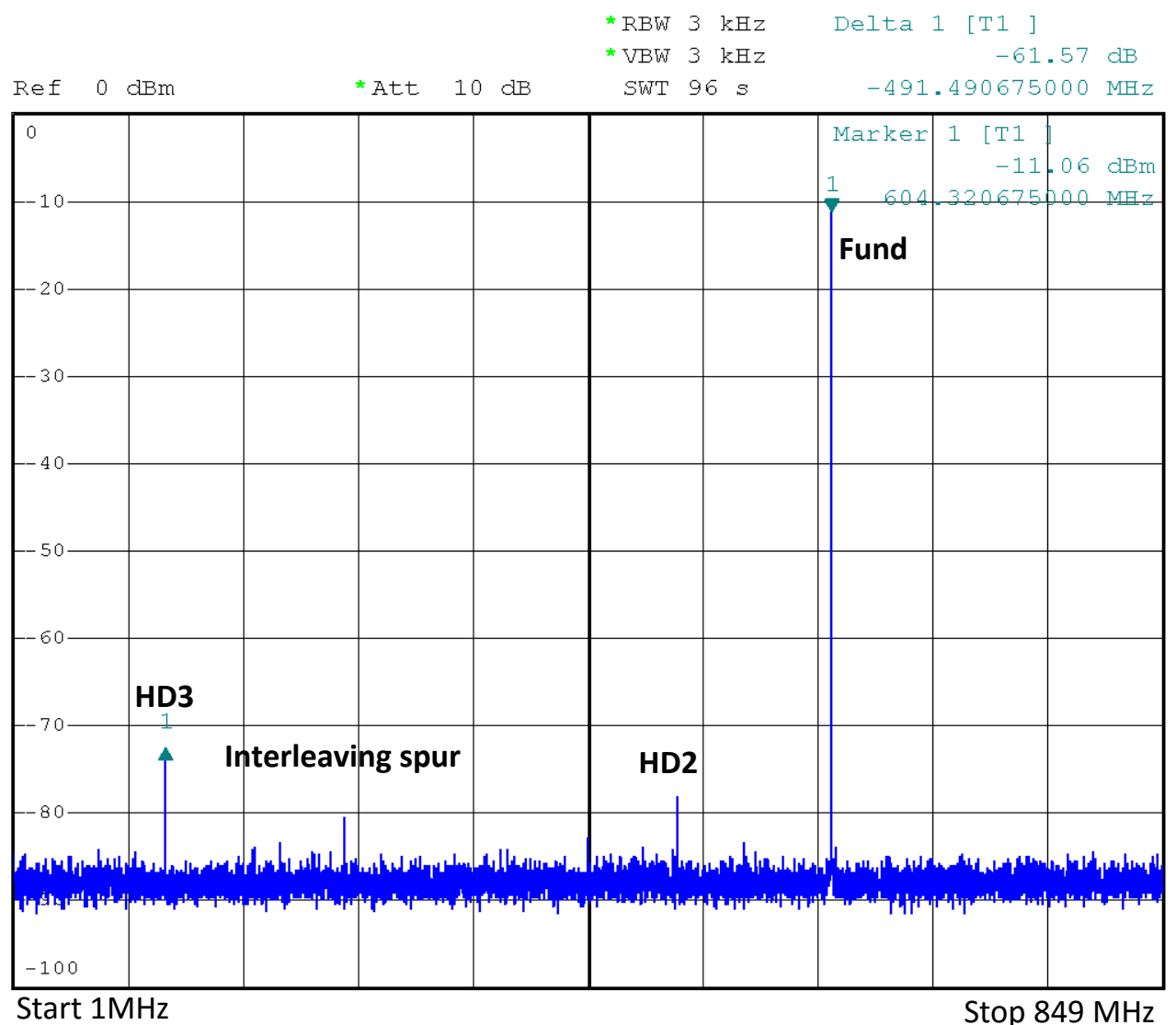

Figure 4-10: Spectral plot with an output frequency of $604 \mathrm{MHz}$ at $1.7 \mathrm{GS} / \mathrm{s}$

This also shows that compared to a non-interleaving DAC the area and power budget does not need to be doubled, since high performance can be achieved with two sDACs that only have moderate performance.

\subsubsection{Low voltage operation}

The interleaved DAC circuit was designed to operate at $1.2 \mathrm{~V}$, and shows only a graceful degradation of performance with lower supply voltages. The median SFDR drops by around $5 \mathrm{~dB}$ when the supply voltage $\mathrm{V}_{\text {supply }}$ is lowered from $1.2 \mathrm{~V}$ to $1 \mathrm{~V}$. Note that we use a resistive load, without the customary RF chokes that would lift the DC level of $V_{\text {out }}$ to $\mathrm{V}_{\text {supply; }}$ consequently the actual voltage at the outputs gets as low as respectively $950 \mathrm{mV}$ and $750 \mathrm{mV}$ with $500 \mathrm{mVpp}$-diff swing during the measurement results presented in this chapter. With the output voltage at $750 \mathrm{mV}$ the majority of this is used to bias the current sources; the interleaving switches are designed to have a low drain- 
source voltage, and also the data switches operate at the edge of triode region when the output voltage decreases to this level.

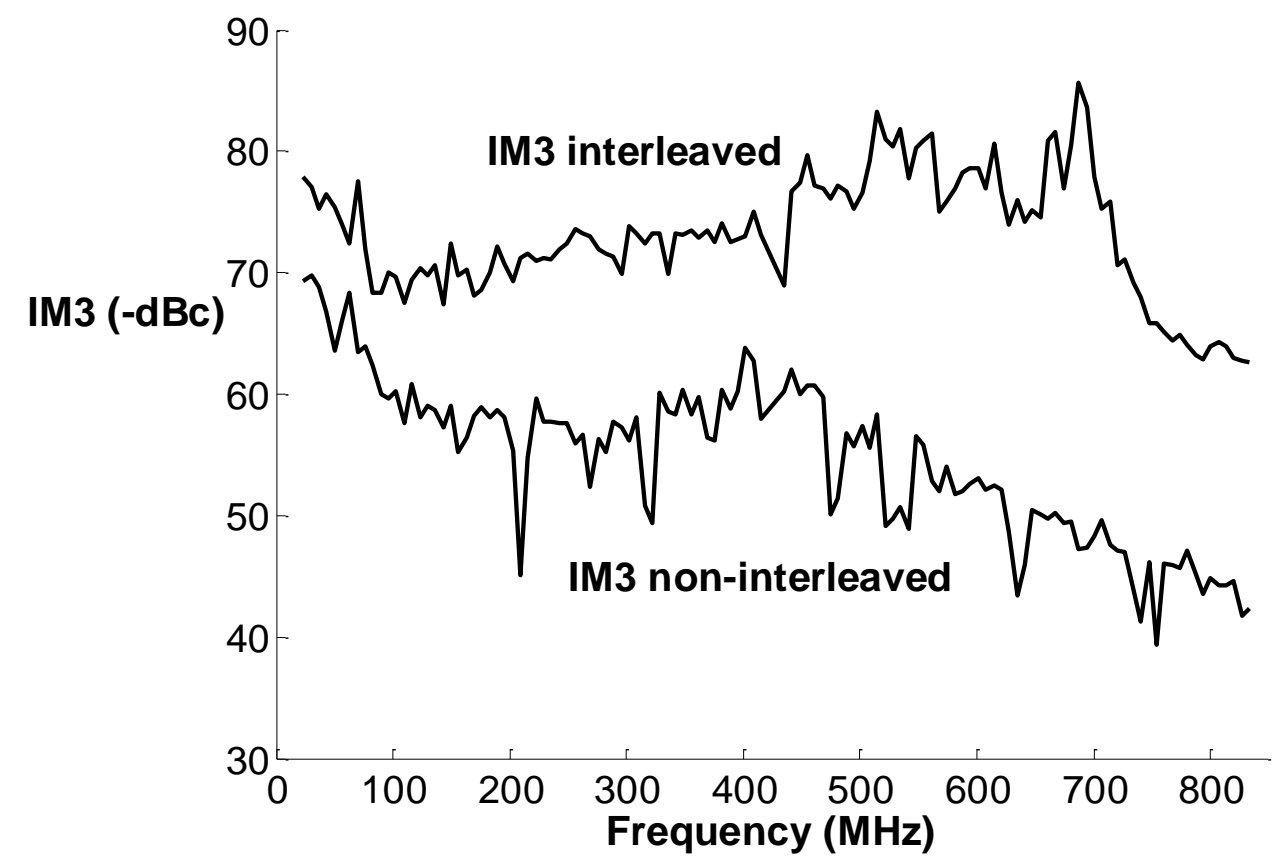

Figure 4-11: IM3 behavior with interleaving enabled and disabled at 1.7GS/s interleaved, $0.85 \mathrm{GS} / \mathrm{s}$ non-interleaved

\subsubsection{Comparison to state-of-the-art}

Figure 4-12 compares to performance obtained by this design with other state-of-theart designs. Compared to the other NRZ results, except [44], this design achieves significantly better SFDR. [22] achieves a better SFDR, but this does utilize return-tozero, which as discussed in section 2.3.2 also has its downsides. While [15] and [34] have a higher sampling rate and output swing, this does come at the cost of a much higher required supply, higher power consumption and a significantly larger core size. [55] is comparable in supply voltage and power consumption, while additionally only requiring a very small area. However its SFDR is significantly worse.

The design of [44] achieves better linearity at a higher sample rate (although SFDR/IM3 is not over its entire Nyquist range) and with a high output current. However with over 
30 times the power consumption and a core area which is more than 10 times larger, the designs are in different categories.

\subsection{Conclusions}

A high-speed interleaving DAC is presented that runs at $1.7 \mathrm{GS} / \mathrm{s}$ at low supply voltages without requiring RF chokes while achieving an SFDR across Nyquist that is better than previous non-return-to-zero DACs with comparable power consumption and does not have the higher clock jitter sensitivity and filtering requirements inherent to an RZ DAC. This interleaving DAC retains the advantages of conventional current steering DACs while the interleaving spurs that may occur when improperly interleaving are effectively suppressed using calibration of the DNL of one sub-DAC, calibrating the difference between the INLs of both sub-DACs to zero and calibrating the timing error to around 210fs. This calibration method can be implemented using low-accuracy, lowspeed AD converters.

\begin{tabular}{|c|c|c|c|c|c|c|}
\hline & This & {$[15]$} & {$[34]$} & {$[22]$} & {$[55]$} & {$[44]$} \\
\hline Tech & $65 \mathrm{~nm}$ & $180 \mathrm{~nm}$ & $65 \mathrm{~nm}$ & $40 \mathrm{~nm}$ & $65 \mathrm{~nm}$ & $180 \mathrm{~nm}$ \\
\hline Resolution [Bits] & 12 & 14 & 12 & 12 & 9 & 14 \\
\hline Power [mW] & 70 & $<600$ & 188 & 40 & 60 & 2300 \\
\hline $\mathrm{V}_{\text {supply }}[\mathrm{V}]$ & 1.2 & $-1.5 / 1.8$ & $1.2 / 2.5$ & 1.2 & 1.2 & $1.8 / 3.3$ \\
\hline Area $\left[\mathrm{mm}^{2}\right]$ & 0.4 & 4 & 0.825 & 0.016 & 0.04 & 5.2 \\
\hline Swing $\left[\mathrm{V}_{\text {pp-diff] }}\right.$ & 0.5 & 1.0 & 2.5 & $0.8(\mathrm{RZ})$ & 0.4 & $80 \mathrm{~mA}^{\mathrm{b}}$ \\
\hline Fs [GHz] & 1.7 & 3 & 2.9 & 1.6 & 3 & 4.6 \\
\hline SFDR $[\mathrm{dB}]$ & 58 & 52 & 52 & 70 & 49 & $69^{\mathrm{c}}$ \\
\hline $\mathrm{IM}^{\mathrm{a}}[\mathrm{dBc}]$ & -62.5 & -65 & -61 & -70 & -60 & $-74^{\mathrm{c}}$ \\
\hline $\mathrm{RZ}$ & No & No & No & Yes & No & No \\
\hline DEM & No & No & No & Yes & No & No \\
\hline
\end{tabular}
a. The worst-case SFDR/IM3 up to Nyquist/850MHz, whichever is lower
b. No load impedance is reported: the voltage swing is unknown
c. SFDR is reported at $500 \mathrm{MHz}, \mathrm{IM} 3$ up to $1 \mathrm{GHz}$

Figure 4-12: Comparison table 



\section{Chapter 5 \\ High-speed Demonstrator: 9- bits at 8.8GS/s and 11GS/s}

This chapter is based on [61], section III and sections V up to VIII. The work in [61] is based on a $28 \mathrm{~nm}$ FDSOI demonstration implementation, which was first published as [66]. In addition to the $28 \mathrm{~nm}$ implementation also a $65 \mathrm{~nm}$ bulk CMOS variant of the same circuit was designed and measured. This circuit was not discussed in [61], but is included in this chapter. Despite the special characteristics of FDSOI technology, it can largely be designed as regular bulk CMOS. Appendix D discusses some of the (potential) advantages FDSOI offers for current steering DACs over bulk CMOS.

Compared to the design discussed in chapter 4 the demonstrators discussed here were designed for higher operating speeds at lower accuracy. Both the $28 \mathrm{~nm}$ and $65 \mathrm{~nm}$ version are 9-bit DACs employing no current source calibration and instead rely on passive current source matching. The target linearity for both designs is above $50 \mathrm{~dB}$ SFDR.

In this chapter, the results of both the $28 \mathrm{~nm}$ and the $65 \mathrm{~nm}$ variant are presented, together with some more in-depth analyses compared to the original paper [61]. The $65 \mathrm{~nm}$ bulk CMOS and the $28 \mathrm{~nm}$ FDSOI implementation are largely identical; the small differences between the two implementations are discussed in section 5.2. 


\subsection{Suppressing code-dependent supply and bias load: Quad- switching}

Due to the switching of the data-switches, both the bias line(s) and power supply line(s) observe a code-dependent load which severely limits DAC linearity. The charge injected into bias and supply lines due to the data-switches results in voltage variations which modulate the output current of each sDAC slice, creating mixer-like behavior. Since the switching action of the data-switches is code-dependent, the resulting voltage variation on the supply and bias nodes is also code-dependent. The mixing of these signals with the intended output signal creates unwanted harmonic distortion components.

The interleaving architecture presented in chapter 3 inherently allows settling of all kinds of switching related issues, as long as sufficient settling is accomplished in an SDAC before placing it on the overall DAC output. For bias and power supply lines this would translate in requiring low-ohmic (reference) voltages without decoupling capacitors as the latter are inherently slow ${ }^{2}$. Compared to the other nodes in highspeed DACs, these bias and supply power lines are however still relatively slow, and a sufficiently low-ohmic reference over a wide bandwidth would require a high power consumption.

Hence, while interleaving suppress artifacts from code-dependent switching, extra measures are required to decrease the code-dependent loading of bias and supply lines in an area and power efficient way.

In the design presented in chapter 4 , the sample rate was sufficiently low to allow bias lines to settle in time using a low-ohmic reference circuit. The higher sampling rate of this design would make the cost in power prohibitive. Chapter 4's design did use decoupling capacitors for the power supply, where the limited bandwidth of the poly capacitors and routing was still acceptable for its 5-6 times lower sampling rate than the designs discussed here.

A well-known method to decrease data-dependent behavior is using dummy structures that switch when the main structure does not switch. This fundamentally comes at the

\footnotetext{
${ }^{2}$ If there are no area requirements, an alternative may be to use very slow settling which effectively will make any bias and power supply line purely DC. This usually requires huge on-chip capacitances and many low-ohmic wiring which typically is not acceptable.
} 
cost of extra power and extra area consumption. Using matched dummy structures to sufficiently suppress loading effects in high-speed DACs, the increase in area and power is roughly a factor 2 .

An alternative to using dummies is quad-switching [41] [15] which uses four switches per current source to direct the current to the positive or negative output, see e.g. figure 5-1. Similar to dummy structures, quad-switches make sure every cycle the same amount of switching activity occurs: regardless of the (change in) code every clock cycle in each SDAC slice one switch will turn off and one switch will turn on. However in contrast to a full dummy structure in parallel, quad-switching re-uses the regular current sources, thereby only adding the extra switches and their drivers, with their corresponding increase in area and active power. Since quad-switching structures can be closely packed, timing matching can be better than with separate dummy structures.

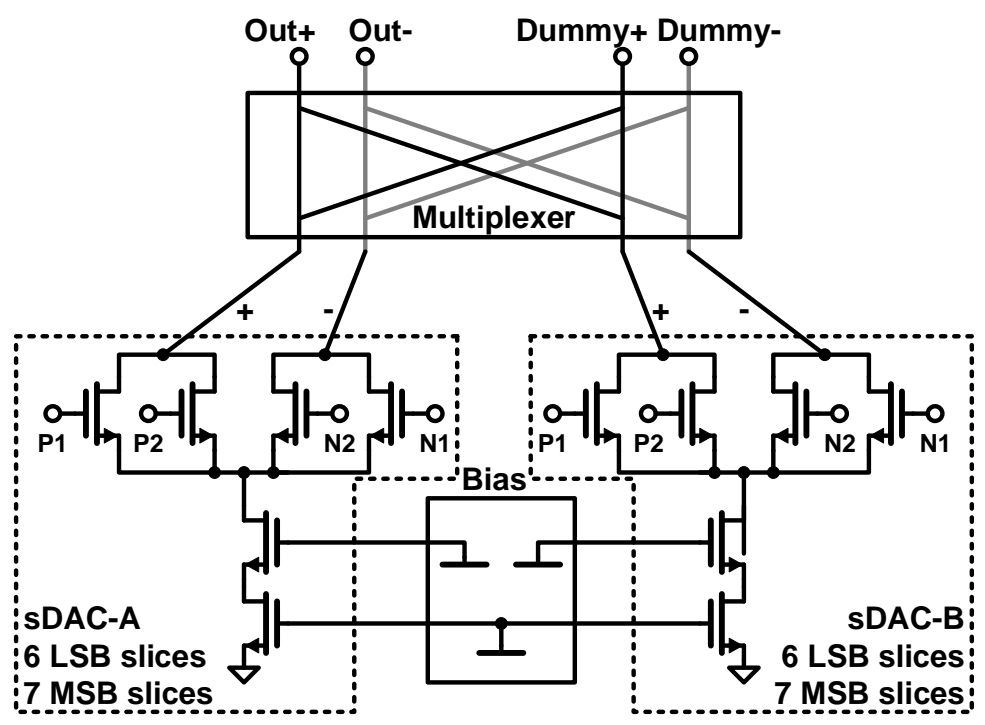

Figure 5-1: Schematic of the SDAC implementation and their connections

However quad-switching also has downsides compared to using dummy structures. The main problem is that the extra switches are directly in the signal path. The extra amount of switches increase the likelihood of timing errors, and differences between the two switches for one side result in spurs similar to interleaving spurs, including the high timing demands of interleaving switches. The usual way to solve these spurs is to run the quad-switches at twice the data rate [42]: in each sample period both switches for the active output are toggled. In interleaving DACs however, these quad-switching 
spurs mentioned above are inherently suppressed in the same way as all other data timing related errors. Static differences in output current between the different quadswitches, for example due to differences in threshold voltage, modulating the drain voltage of the current source, are suppressed by the output impedance of the current sources, and are generally not an issue. This makes quad-switching very suitable to be integrated in an interleaved architecture: the advantages of quad-switching are obtained, while its disadvantages are inherently suppressed.

\subsection{Circuit implementation details}

The interleaving architecture significantly simplifies the sDAC design: since most of the dynamic errors are suppressed, the design is not as critical as that of regular DACs. As discussed in section 3.1.1, static errors are not suppressed by interleaving but do not have to limit performance at medium resolution and high-speed.

Figure 5-1 shows the implementation of the sDACs and their connections. Both of the 9-bit sDACs consist of 6 binary coded LSBs, and 3 thermometer coded MSBs. Quadswitches redirect their output current to either the positive or the negative output. The quadrature generation and switch drivers are implemented with standard core cells, this sets the minimum the width of each sDAC slice. The driver power of these standard cells limit the width of the data switches that can be driven by one slice. Placing standard cells in parallel to increase the drive power is possible, but makes the layout of the smaller slices less efficient. The maximum drive power of one slice, combined with the preference to use as few slices as possible to reduce area and power, sets the segmentation of the design: 6 bits are binary implemented, while the remaining 3 bits are thermometer coded. The current source transistors of the two sDACs share a single bias rail to improve their matching, while the bias lines of the cascode transistors are only shared within an SDAC to improve isolation between the two sDACs.

Dynamic errors are suppressed as long as they are sufficiently died out by the time the sDAC is connected to the output: for that, all internal nodes need to be sufficiently settled. For errors such as sub-picosecond timing mismatch in the data switches this is not a problem, and they will be virtually completely suppressed. For measurements, the demonstrator chips are packaged in a standard QFN32 package. While the four $50 \Omega$ single-ended on-chip resistors in addition to the four $50 \Omega$ off-chip resistors required for measurements reduce the influence of the bondwires, the bondwire inductances still prevents complete settling of the output nodes within a sampling period. 
During operation, the SDAC that is switched to a new code is not connected to the DAC output. Ideally the errors during this settling are fully suppressed in the interleaving architecture. However, these settling related errors before the SDAC is placed at the output can still propagate to the output: the multiplexer switches do not provide $100 \%$ isolation, especially for larger current spikes. Also variation in the on-resistance of the multiplexer switches, due to e.g. mismatch/proximity effects and due to drain current dependencies may yield spurs in the DAC output. These latter effects decrease with increasing SDAC output impedance.

\subsubsection{Current sources}

The current sources in the sDACs need to have a sufficiently high output impedance and they must be well-matched. These demands are partialconflicting. Good matching implies that the transistors must be relative large and preferably are in a commoncentroid layout. For large transistors, wide and/or long transistors must be used. Wide transistors result in low output impedances, while long transistors result in a low voltage headroom. Increasing matching using e.g. common-centroid techniques usually increases the total output capacitance of current sources. In our $9 \mathrm{~b}$ design, the current sources are placed in a common-centroid layout. The common-centroid layout is required to fulfill matching requirements. The 12-bit design discussed in chapter 4 employed active calibration, which removed this matching requirement for that design. The cascode transistors are grouped together with the quad-switches and their drivers in a line-layout to minimize parasitics, see figure 5-2. This allows the current sources to be optimized for matching and output resistance, with a small cascode transistor to obtain low parasitic capacitances.

In the $28 \mathrm{~nm}$ FDSOI design this setup also resulted in sufficient inter-sDAC matching to meet the amplitude error requirements. The larger mismatch between the sDACs in the $65 \mathrm{~nm}$ design is compensated for by variable cascode bias voltages. The bias voltage of the cascodes of the two sDACs can be independently adjusted per sDAC. This modulates the drain voltage of the current sources, which modulates their current output due to their finite output impedance. 


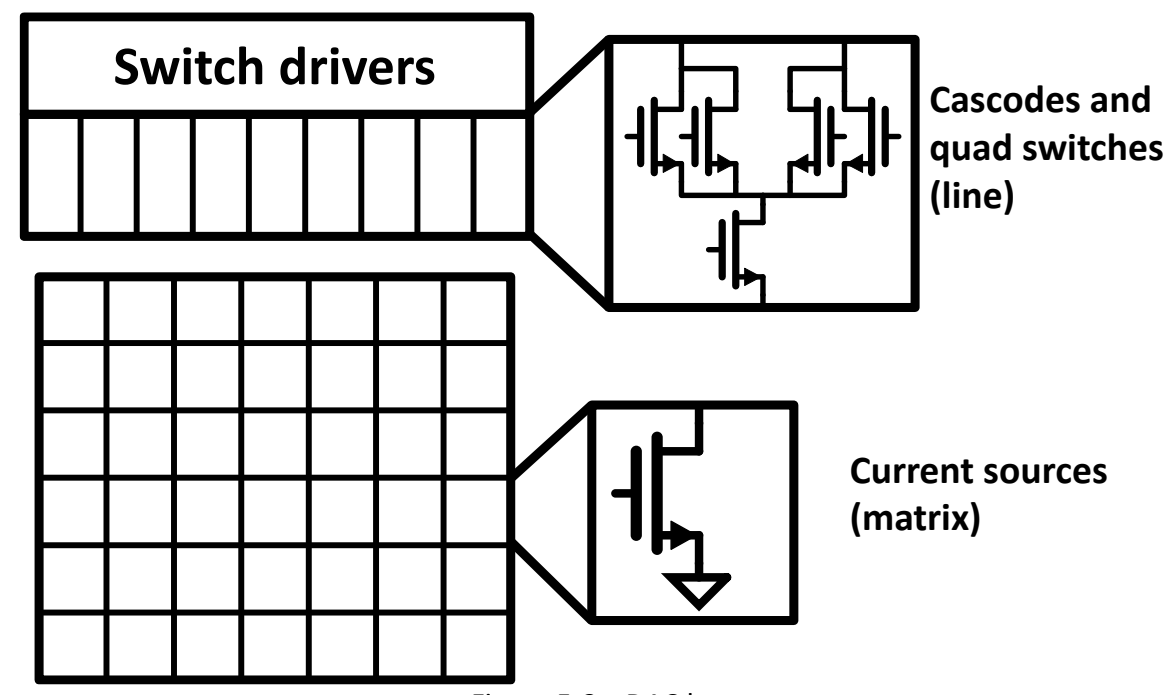

Figure 5-2: sDAC layout

\subsubsection{Switch drivers and signal generation}

Proper switch signals for the quad-switching architecture are generated and buffered in the interleaved DACs. The interleaving architecture inherently suppresses the propagation of switching related errors to the DAC output which lowers the demand on generation of the switch signals. Both current-mode logic (CML) drivers and CMOS circuitry can be used to drive the quad-switches [34, 55]; both having specific advantages and disadvantages. Quad-switching combined with interleaving removes the need for most of the CML advantages, and using CML in combination with quadswitching is more complicated than using CMOS drivers. In our designs, standard digital core cells are used to generate and buffer switch signals. These are area and power efficient, at the cost of a less than ideal switching waveform. Only in the $65 \mathrm{~nm}$ implementation the final set of latches to re-time the switch signals, drawn at the right side in figure 5-3, are implemented using custom dynamic latches instead of regular core cell latches. Dynamic latches require a clock signal to maintain their state; this clock can be combined with/derived from the clock that is required for the analog multiplexer. Compared to a regular core cell latch, the implemented dynamic latch obtained better speed and lower delays, which were required for the $65 \mathrm{~nm}$ design to meet its stringent timing constraints.

Figure 5-3 shows the logic necessary to generate the quad-switching waveforms. The input clock is divided by two to generate two opposite clock signals at half the main clock frequency. A logical AND gate is used between those half-rate clocks and the 
data/inverted data. This results in four outputs, of which only one can be high at a time. The half-rate clocks ensures that it is never possible that one switch signal is high for more than one single consecutive sDAC period.

The quad-switches themselves perform effectively a logical OR operator: P1 OR P2 are equal to Data, while N1 OR N2 are equal to inverted Data. Different propagation delays of the used cells can result in small glitches in the outputs of the AND gates. The retiming latches suppress these glitches and output a clean signal to the switch buffers.

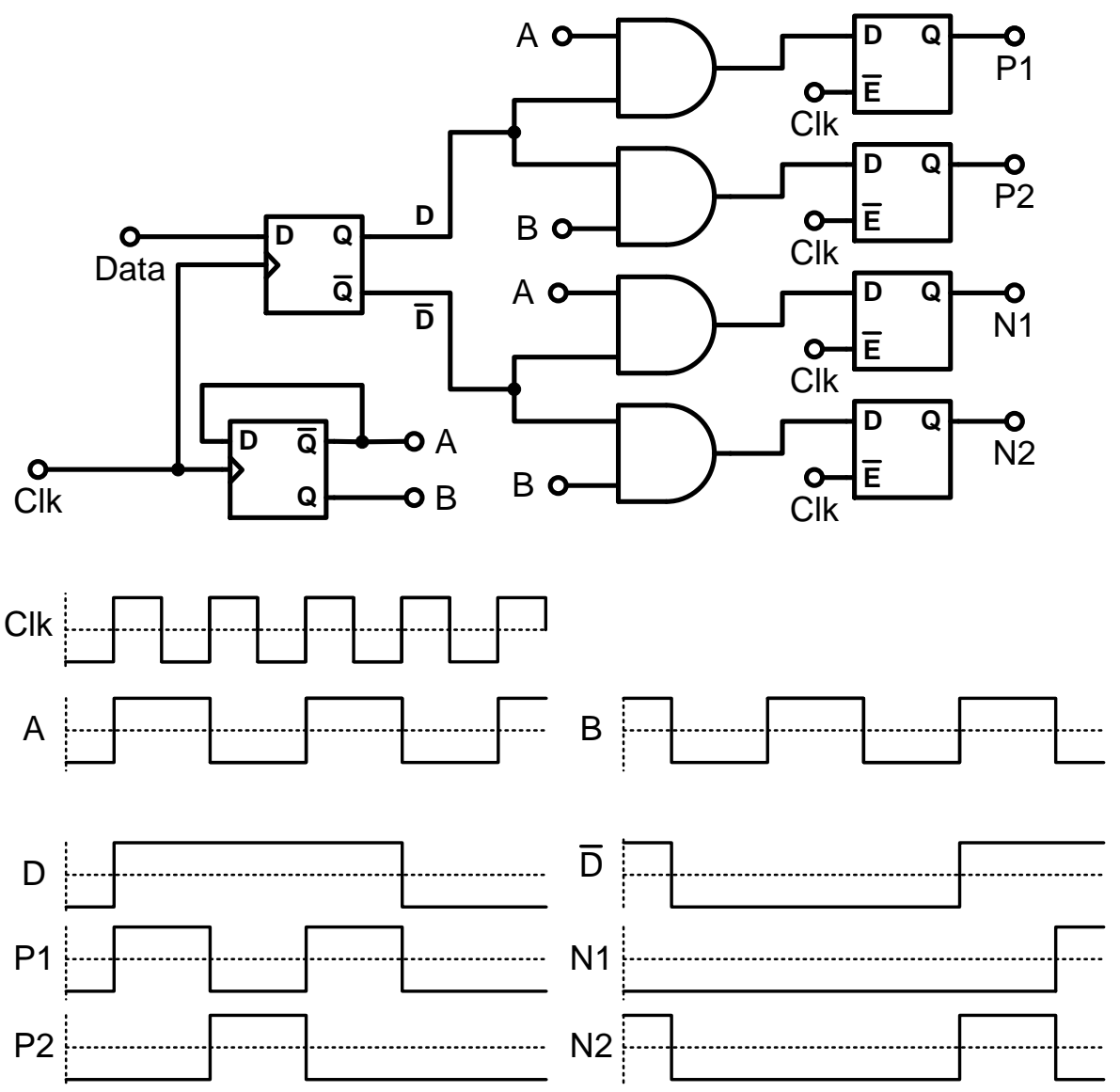

Figure 5-3: Quad-switching signal generation and waveforms

\subsubsection{Multiplexer and driver design}

The analog multiplexer combines the two sDACs into a single DAC. This multiplexer consists of 8 NMOS transistors in total, to direct the differential current of the two 
sDACs to either the real output or the dummy output. In their on-state they are in triode, and are dimensioned such that they add just a few ohms series resistance. This series resistance in series with the $25 \Omega$ load resistance (the $50 \Omega$ off-chip load parallel to the $50 \Omega$ on-chip load), ensures that both the voltage drop and the variation in the total resistance seen by the sDACs is sufficiently small.

To drive the multiplexer transistors into triode region a capacitive level shifter is used. This is done using the same setup as implemented in the design of chapter 4 and shown in figure 4-2.

Tuning of the duty cycle to get a sufficient SFDR can be done in multiple ways. For example the design discussed in chapter 4 ( [65] ) uses an array of capacitors followed by an SR latch to adjust the duty cycle. In our $28 \mathrm{~nm}$ FDSOI design, the backgate of the FDSOI multiplexer transistors themselves are connected to a variable voltage source. The wells for the transistors driven by SW+ are shared, as are those driven by SW-. Changing the backgate voltage modulates the threshold voltage of the multiplexer transistors which changes the turn-on and turn-off speed of the corresponding transistors which efficiently implements duty cycle tuning. Since there is no junction between the well and the source and drain areas, the backgates are allowed to be tuned between $0 \mathrm{~V}$ and $3 \mathrm{~V}$, which implies a differential tuning range of $-3 \mathrm{~V}$ to $3 \mathrm{~V}$.

The NMOS multiplexer transistors in the $65 \mathrm{~nm}$ technology are in a regular PWELL, which needs to be connected to ground. Deep-NWELLs can be used to isolate a PWELL and connect it to another potential, but this would complicate the design and increase the size of the multiplexer.

The chosen alternative for duty cycle tuning in 65nm bulk CMOS is shown in figure 5-4. Instead of modifying the threshold voltage of the multiplexer transistors themselves, the threshold of two PMOS transistors in the buffer can be adjusted. This increases/decreases the slew rate of the rising edge of the output signal of the leftmost inverter, which in turn increases/decreases the duty cycle of the drive signal for the subsequent inverters that ensure maximum slew rate at the multiplexer inputs.

The $65 \mathrm{~nm}$ CMOS technology is a bulk process and hence diodes are present between bulk and the drain/source nodes. For this reason the bulk voltage may not be significantly below the power supply voltage. 


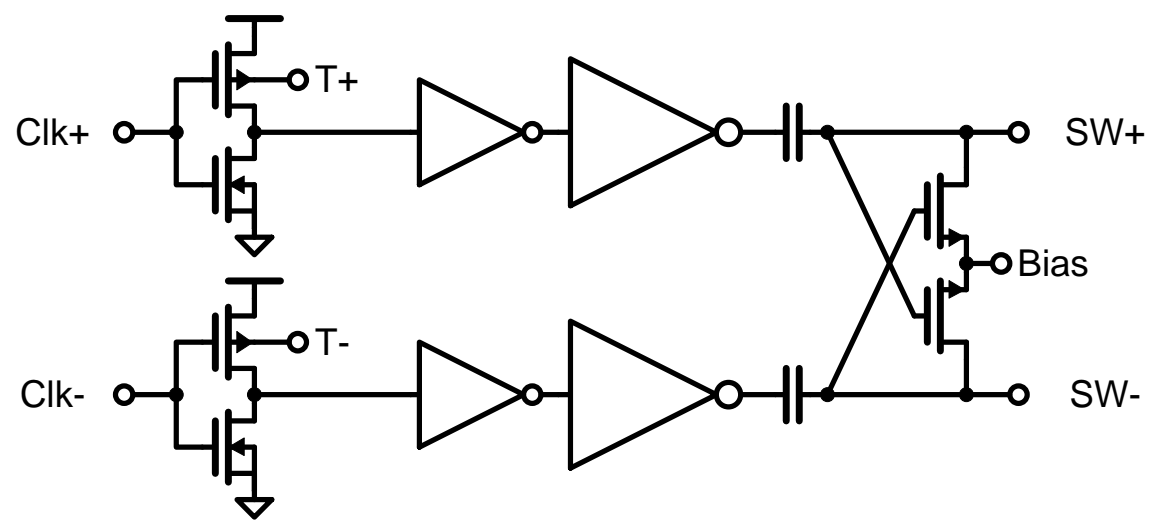

Figure 5-4: Multiplexer driver in the $65 \mathrm{~nm}$ to facilitate duty cycle adjustments.

\subsection{Demonstrator chip}

A 9-bit interleaved DAC was built in 28nm FDSOI CMOS technology; figure 5-5 shows the die photograph, with 6 binary segmented bits and 3 thermometer bits. The quadrature generation and switch drivers are implemented with standard core cells; together with the switches themselves and the requirement to have each cell as narrow as possible, this limits the maximum current that can properly be switched by each cell.

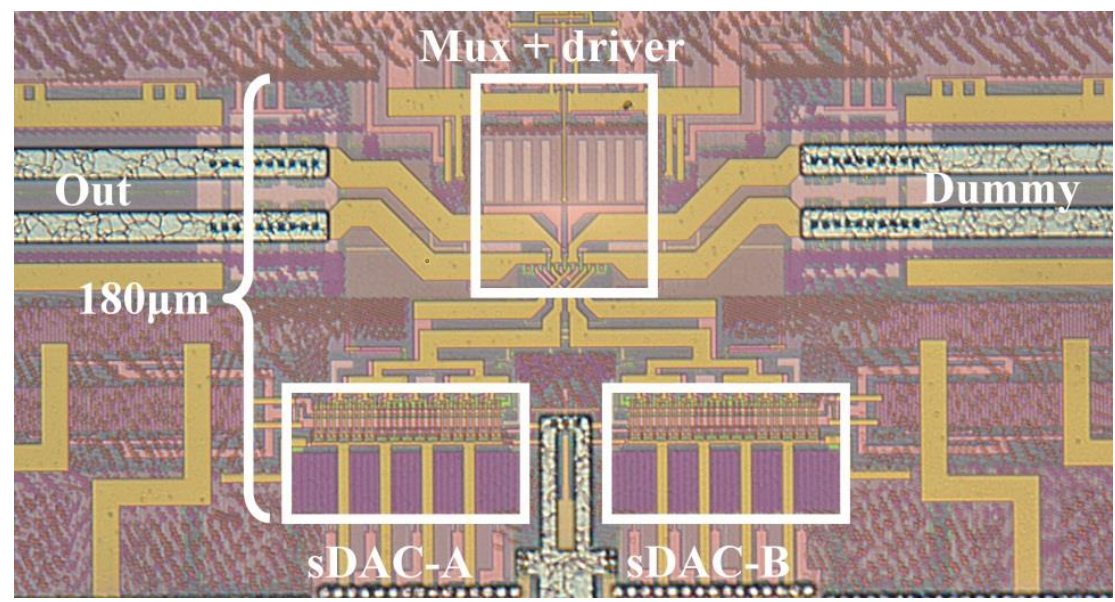

Figure 5-5: Die photograph of the DAC core in $28 \mathrm{~nm}$ FDSOI; the core dimensions are $180 \mu \mathrm{m}$ by $220 \mu \mathrm{m}$ 


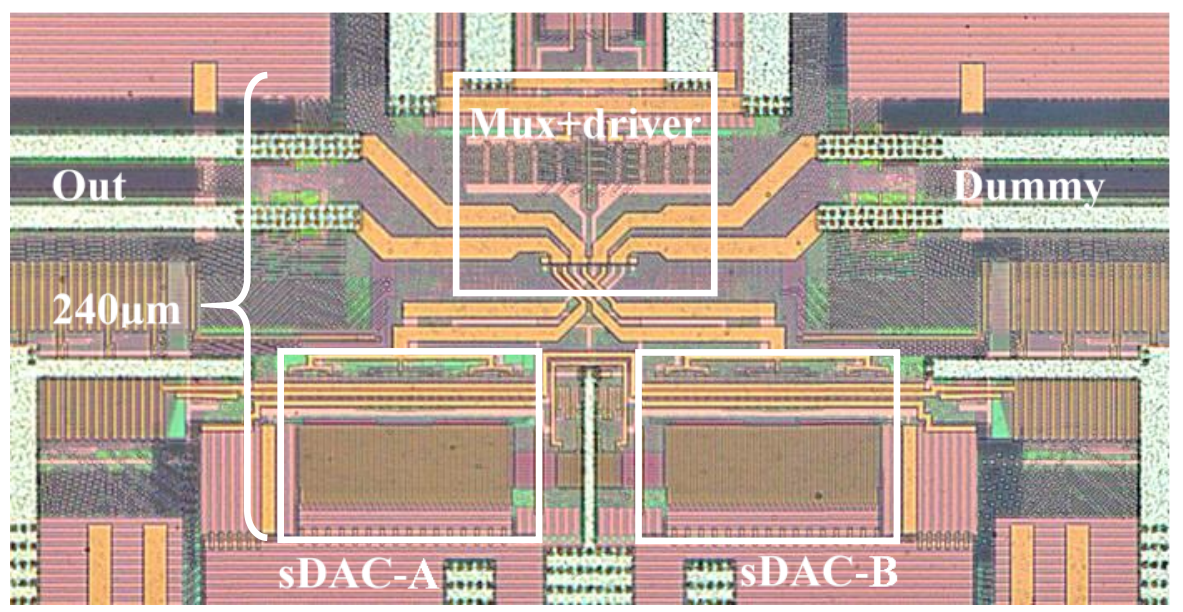

Figure 5-6: Die photograph of the DAC core in $65 \mathrm{~nm}$ bulk CMOS; the core dimensions are $240 \mu \mathrm{m}$ by $310 \mu \mathrm{m}$

Compared to the DAC one discussed in chapter 4, these DACs operate at a much higher frequency, which makes implementing a digital sine generator such as used for the DAC from chapter 4 unrealistic. The implemented alternative is a 63-word memory for each SDAC that provides a combined 126 word memory for test signals. Synchronization logic makes sure that after programming the memory it will be read out in the correct order. The $28 \mathrm{~nm}$ implementation has an on-chip clock divide-by-two block which isolates the internal duty cycle from possible external distortion components. For the $65 \mathrm{~nm}$ version this block is omitted due to speed constraints, and instead it relies on CML clock buffers to maintain a stable duty cycle. The memory architecture is further discussed in Appendix B.

The sDACs in the $28 \mathrm{~nm}$ FDSOI implementation occupy approximately $0.006 \mathrm{~mm}^{2}$ each, while the total DAC core area is $0.04 \mathrm{~mm}^{2}$. For the $65 \mathrm{~nm}$ implementation the SDAC area is approximately $0.011 \mathrm{~mm}^{2}$ while the total DAC core occupies $0.075 \mathrm{~mm}^{2}$, so almost a factor two more than the $28 \mathrm{~nm}$ version. While the unit current sources are fairly equal in size between the two implementations, everything that switches is scaled to minimum length in $28 \mathrm{~nm}$, which in turn can reduce the width while keep the transconductance equal. A significant part of the sDACs are the standard core cells that control the data switches; these are a lot smaller in $28 \mathrm{~nm}$ compared to $65 \mathrm{~nm}$. The $28 \mathrm{~nm}$ DAC, excluding memory, consumes $110 \mathrm{~mW}$ from a single $1.0 \mathrm{~V}$ power supply source at its nominal speed of $11 \mathrm{GS} / \mathrm{s}$, while the $65 \mathrm{~nm}$ DAC consumes $100 \mathrm{~mW}$ from its $1.2 \mathrm{~V}$ supply at $8.8 \mathrm{GS} / \mathrm{s}$. For measurement purposes the output is biased with a DC choke at 
$0.9 \mathrm{~V}$ for the $28 \mathrm{~nm}$ test setup and $1.0 \mathrm{~V}$ for the $65 \mathrm{~nm}$ test setup. A signal swing of $425 \mathrm{mVpp}$-diff at DC was used during testing for the $28 \mathrm{~nm}$ demonstrator, while the $65 \mathrm{~nm}$ version used $400 \mathrm{mVpp}$-diff swing. The extra headroom for the $65 \mathrm{~nm}$ chip (a slightly reduced swing combined with higher supply voltage) allowed it to extend its better than $50 \mathrm{~dB}$ SFDR to its entire Nyquist range.

\subsection{Measurements}

All measurements were performed at the nominal settings described in the previous section and after timing calibration. Timing calibration was performed manually using the DC timing measurement method outlined in section 3.2, however it could have been easily automated. Figure 5-7 shows the measured output spectrum for a single tone full scale signal at $4.6 \mathrm{GHz}$ for the $28 \mathrm{~nm}$ implementation with an $11 \mathrm{GS} / \mathrm{s}$ sample rate, and figure $5-8$ the output spectrum of the $65 \mathrm{~nm}$ implementation of a $3.7 \mathrm{GHz}$ fundamental and a sample rate of $8.8 \mathrm{GS} / \mathrm{s}$, which for both setups is slightly above $80 \%$ of their Nyquist frequency. Figure 5-9 shows the measured SFDR and IM3 versus output frequency for the $28 \mathrm{~nm}$ demonstrator, while figure 5-10 shows the similar spectrum for the $65 \mathrm{~nm}$ demonstrator. For each of the designs, both the SFDR and IM3 stay respectively above $50 \mathrm{~dB}$ and below $-50 \mathrm{dBc}$ across Nyquist after timing calibration. At Nyquist, the fundamental is about $5 \mathrm{~dB}$ lower than at $\mathrm{DC}$ due to mainly the sinc roll-off of the zeroth-order-hold.

Since the nonlinearities are largely due to the multiplexer transistors, the HD3 scales with $3 \mathrm{~dB}$ per $\mathrm{dB}$ increase in the fundamental and the HD2 tones scale with $2 \mathrm{~dB}$ per $\mathrm{dB}$. This behavior is expected, since the interleaving mechanism limits the harmonics generated by the sDACs. Due to this relationship, the amplitude which can be obtained with an interleaved DAC with the multiplexer transistors residing in triode is limitted. Such a limitation does not exist for the output current, and by proper impedance scaling it is possible to increase the output power by delivering more current into a lowerohmic output node, e.g. via a transformer. 


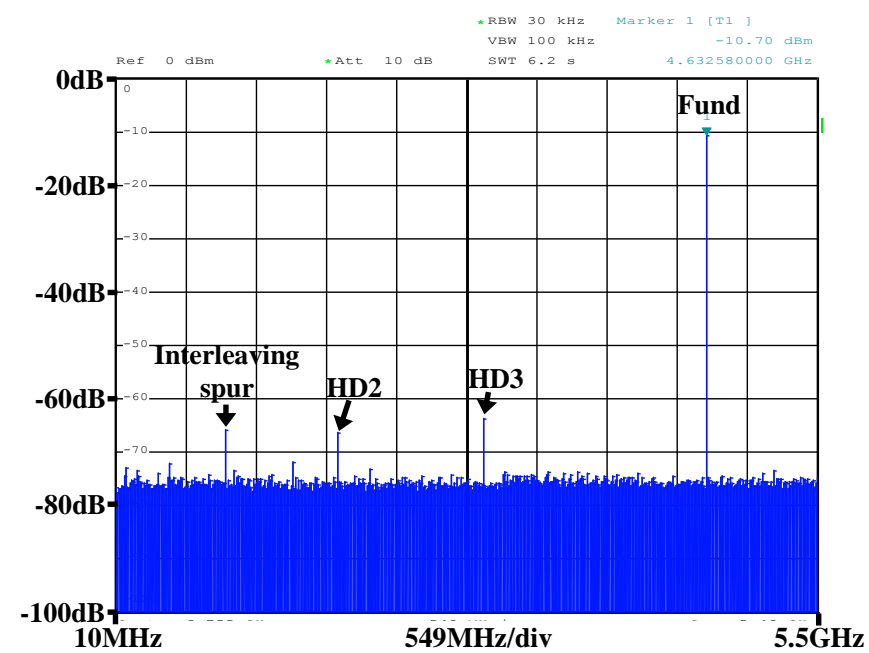

Figure 5-7: Measured output spectrum of the $28 \mathrm{~nm}$ demonstrator with $4.6 \mathrm{GHz}$ full scale sine output across Nyquist at $11 \mathrm{GS} / \mathrm{s}$

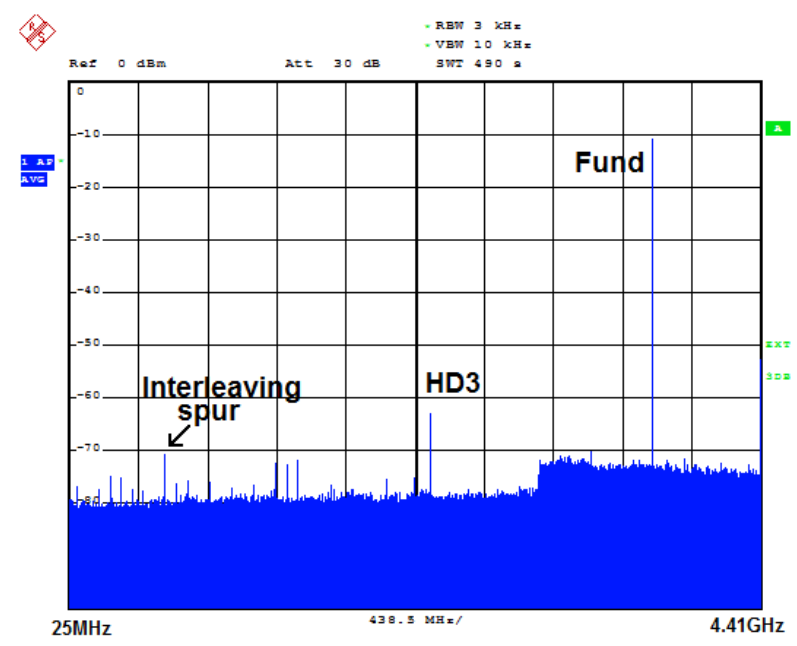

Figure 5-8: Measured output spectrum of the $65 \mathrm{~nm}$ demonstrator with $3.71 \mathrm{GHz}$ full scale sine output across Nyquist at $8.8 \mathrm{GS} / \mathrm{s}$ 


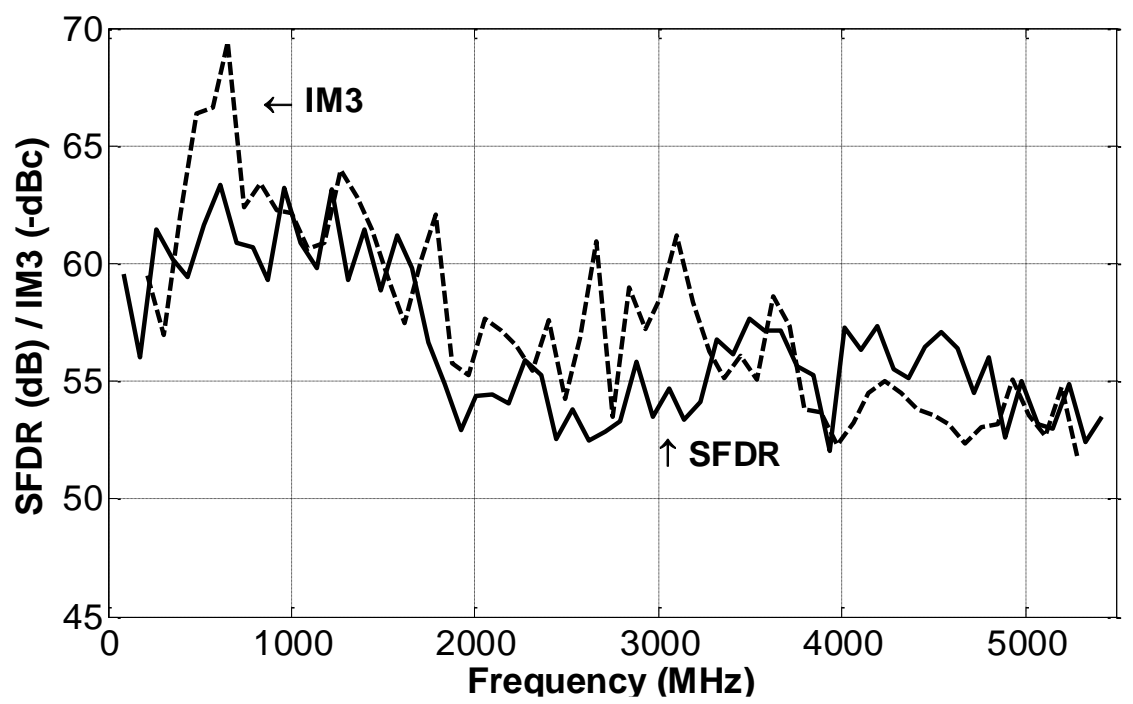

Figure 5-9: SFDR and IM3 versus output frequency for the $28 \mathrm{~nm}$ FDSOI IC at $11 \mathrm{GS} / \mathrm{s}$

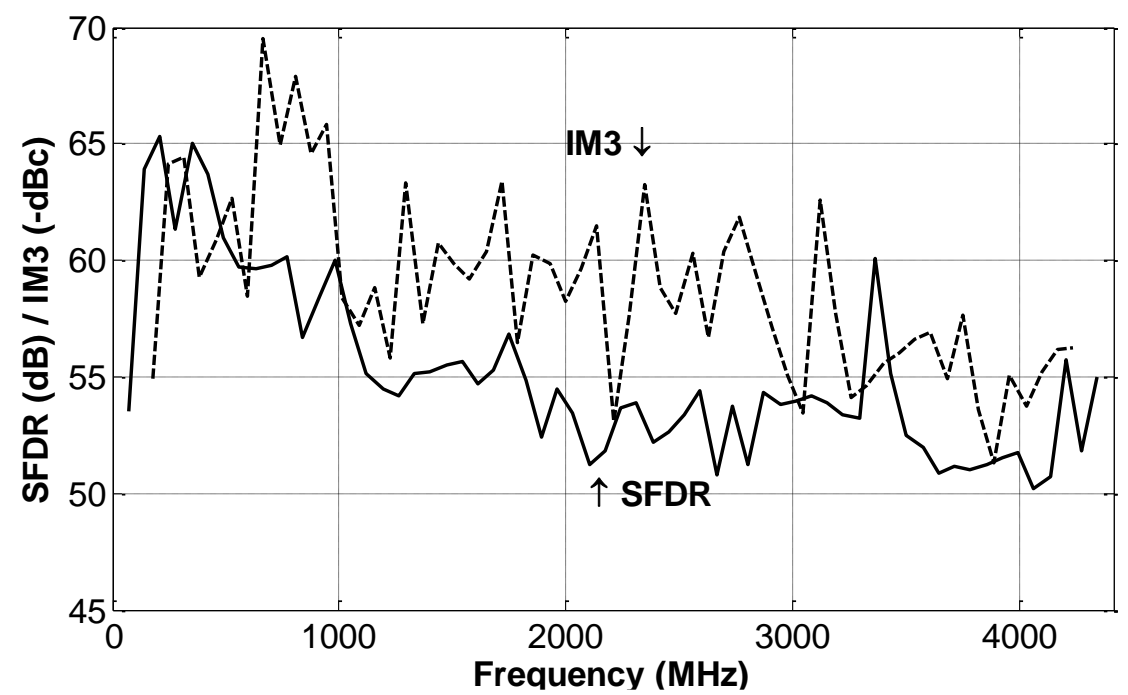

Figure 5-10: SFDR and IM3 versus output frequency for the $65 \mathrm{~nm} \mathrm{IC} \mathrm{at} 8.8 \mathrm{GS} / \mathrm{s}$

The sensitivity of the timing adjustment was determined using the DC timing measurement discussed in section 3.2. In the $28 \mathrm{~nm}$ FDSOI CMOS processes, the tuning range of the back gates is larger than that of transistors in bulk CMOS, and is more linear. Figure 5-11 shows the measured timing for both the $28 \mathrm{~nm}$ FDSOI implementation and the $65 \mathrm{~nm}$ bulk implementation. Both implementations used a 
tuning range of $-2 \mathrm{~V}$ to $2 \mathrm{~V}$ differential. The $28 \mathrm{~nm}$ FDSOI chip can accept single-ended voltages of $\mathrm{OV}$ to $3 \mathrm{~V}$, however above $2 \mathrm{~V}$ linearity starts to degrade due to a too low threshold voltage for proper operation. The $65 \mathrm{~nm}$ implementation accepts $1 \mathrm{~V}$ to $3 \mathrm{~V}$ inputs, resulting in the same differential voltage range. The better linearity of the $28 \mathrm{~nm}$ FDSOl timing tuning is visible, however the used implementation of the $65 \mathrm{~nm}$ chip results in a larger tuning range. The sensitivity of the timing adjustment is of the $28 \mathrm{~nm}$ FDSOI design is $1.2 \mathrm{ps} / \mathrm{V}$, while the $65 \mathrm{~nm}$ design has a sensitivity of $1.75 \mathrm{ps} / \mathrm{V}$. The samples measured for figure 5-11 have an uncalibrated error of 500fs and $350 \mathrm{fs}$ for respectively the $28 \mathrm{~nm}$ FDSOI and the $65 \mathrm{~nm}$ implementation. The measured average and standard deviation the timing error are respectively $0.65 \mathrm{ps}$ and $0.75 \mathrm{ps}$ of the $28 \mathrm{~nm}$ FDSOI design (based on 21 samples) and $1.2 \mathrm{ps}$ and $0.95 \mathrm{ps}$ of the $65 \mathrm{~nm}$ design (based on 23 samples). The error due to amplitude imbalance is included in this: it reflects the timing shift required to fully cancel the interleaving spur at Nyquist.

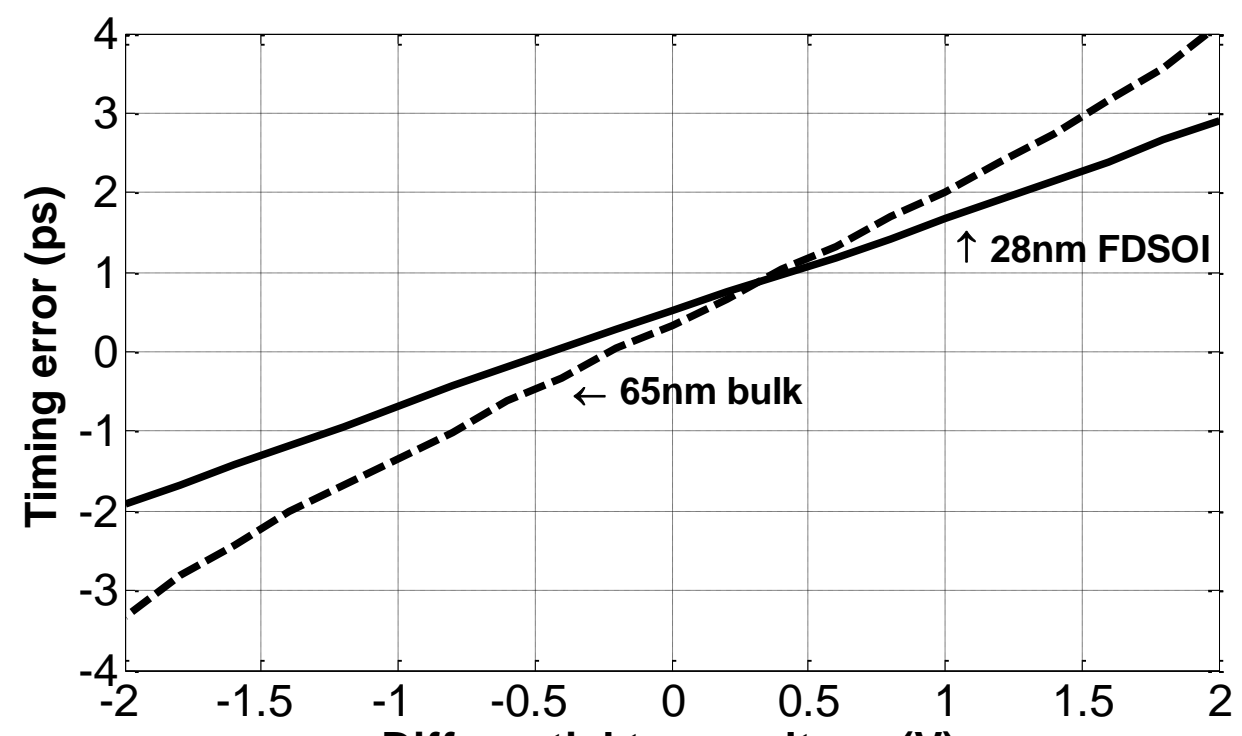

Differential tune voltage (V)

Figure 5-11: Timing error for different tune voltages for the $28 \mathrm{~nm}$ and $65 \mathrm{~nm}$ demonstrator designs

The gain error between the 2 sDACs of the $28 \mathrm{~nm}$ demonstrator sample shown in the measurement plots above was roughly 1.4LSB on the full scale output. According to (34) this should result an image spur with a fundamental close to DC of $61 \mathrm{~dB}$. The measured interleaving spur of this $28 \mathrm{~nm}$ chip was at $-63.5 \mathrm{dBc}$ for a fundamental close 
to $D C$, which is a little better than the theoretical value due to the extra suppression of the interleaving spur when the fundamental is close to DC; the interleaving spur is in this situation located near Nyquist where bandwidth limitations will play a role. After timing calibration, the interleaving spur does not limit performance over Nyquist. The $28 \mathrm{~nm}$ demonstrator achieved a worst-case interleaving spur after timing calibration at $-55 \mathrm{dBc}$ while it was $-60 \mathrm{dBc}$ at Nyquist. The $65 \mathrm{~nm}$ demonstrator's measured worst-case interleaving spur after calibration was located at Nyquist and equal to $-55 \mathrm{dBc}$.

\subsubsection{Comparison to state-of-the-art}

Figure 5-12 shows a summary of the performance and a comparison with state-of-theart. Most notable in the comparison are the power consumption and the required area; these are significantly lower than for most comparable designs. [55] targets similar specifications as those shown in this chapter; they are all 9-bit, the signal swing is identical and while [55]'s IM3 is better, its SFDR numbers are slightly worse. It requires the same area as the $28 \mathrm{~nm}$ version, so it is smaller than the $65 \mathrm{~nm}$ version, and it also requires less power. Summarized it is in those specs slightly better, the demonstrators presented in this chapter operate at 3-4 times the clock and signal frequency compared to the design of [55].

The work in [35] has high output power (at an unknown impedance level) and a similar clock speed. However this comes at the cost of a higher supply voltage, higher power consumption and a much larger die area while obtaining worse linearity. [44] is in a similar situation, only it has much better SFDR/IM3, however this is only reported up to a very limited frequency (respectively $500 \mathrm{MHz}$ and $1 \mathrm{GHz}$ ). Also the factor 20 in power and factor 100 in area shows this comes at a high penalty.

Just like [44], also [15] is a 14-bit design, which did publish its linearity up to Nyquist. However despite the area and power invested in the 14-bit architecture and lower sample rate, only in IM3 its linearity is better than our 9-bit design.

Finally [67] is an impressive design that reaches 56GS/s. It also does this with a relative small and straightforward design, however at lower frequencies its 6-bit architecture cannot deliver the performance of our designs.

\subsection{Conclusions}

Two 9-bit DACs are presented that uses two-times interleaving to obtain an SFDR above $50 \mathrm{~dB}$ across Nyquist and an IM3 below $-50 \mathrm{~dB}$ across Nyquist. The $65 \mathrm{~nm}$ demonstrator 
runs at a nominal speed of $8.8 \mathrm{GS} / \mathrm{s}$ and draws $100 \mathrm{~mW}$ from its $1.2 \mathrm{~V}$ supply, while the $28 \mathrm{~nm}$ demonstrator runs at $11 \mathrm{GS} / \mathrm{s}$ while drawing $110 \mathrm{~mW}$ from a $1 \mathrm{~V}$ supply. These two chips demonstrate that despite requiring two CS DACs in parallel and a multiplexer to combine those, the decrease in demands on the sDACs can result in an overall small and power-efficient high-speed DAC. Compared to state-of-the-art the measured SFDR is equivalent and the IM3 is a bit worse. However the power consumption is a lot lower and also the core area, $0.04 \mathrm{~mm}^{2}$ and $0.074 \mathrm{~mm}^{2}$ for respectively the $28 \mathrm{~nm}$ demonstrator and the $65 \mathrm{~nm}$ demonstrator, is much smaller in this design. Triode switches and quad-switching yield additional reduction in demands on the power supply and bias generation, which allows a decrease in power and area while maintaining good linearity.

\begin{tabular}{|c|c|c|c|c|c|c|c|c|}
\hline & This & This & {$[35]$} & {$[15]$} & Chapter 4 & {$[55]$} & {$[67]$} & {$[44]$} \\
\hline Tech & $\begin{array}{c}28 \mathrm{~nm} \\
\text { FDSOI }\end{array}$ & $65 \mathrm{~nm}$ & $28 \mathrm{~nm}$ & $180 \mathrm{~nm}$ & $65 \mathrm{~nm}$ & $65 \mathrm{~nm}$ & $65 \mathrm{~nm}$ & $180 \mathrm{~nm}$ \\
\hline $\begin{array}{c}\text { Resolution } \\
{[\text { Bits] }}\end{array}$ & 9 & 9 & 13 & 14 & 12 & 9 & 6 & 14 \\
\hline Power [mW] & 110 & 100 & 375 & $>600$ & 70 & 60 & 750 & 2300 \\
\hline Vsupply [V] & 1.0 & 1.2 & 1.8 & $-1.5 / 1.8$ & 1.2 & 1.2 & $1.1 / 2.5$ & $1.8 / 3.3$ \\
\hline Area [mm2] & 0.04 & 0.075 & 1.16 & 4 & 0.4 & 0.04 & 0.24 & 5.2 \\
\hline $\begin{array}{c}\text { Swing } \\
{[\text { Vpp-diff] }}\end{array}$ & 0.425 & 0.4 & $13.5 \mathrm{dBm}^{\mathrm{b}}$ & 1.0 & 0.5 & 0.4 & 0.6 & $80 \mathrm{~mA}^{\mathrm{b}}$ \\
\hline Fs [GHz] & 11 & 8.8 & 9 & 6 & 1.7 & 3 & 56 & 4.6 \\
\hline SFDR $^{\mathrm{a}}[\mathrm{dB}]$ & 51 & 50 & - & 52 & 58 & 49 & 43 & $69^{\mathrm{c}}$ \\
\hline $\mathrm{IM3}^{\mathrm{a}}[\mathrm{dBc}]$ & -51 & -52 & -44 & -65 & -62.5 & -60 & - & $-74^{\mathrm{c}}$ \\
\hline
\end{tabular}

a) Worst-case reported SFDR/IM3 up to Nyquist/5.5GHz

b) No load impedance is reported: the voltage swing is unknown

c) SFDR is reported at $500 \mathrm{MHz}, \mathrm{IM} 3$ up to $1 \mathrm{GHz}$

Figure 5-12: Comparison table 


\section{Chapter 6 \\ Conclusions}

\subsection{Summary and conclusions}

Chapter 1 presented an introduction into high-speed DACs, including the basics of digital-to-analog conversions and the reasons that faster and more accurate DACs are required. Different performance figures relevant to the presented DACs were introduced. Finally in this chapter different types of DACs and their operation principles were discussed.

This is followed in chapter 2 by a more in-depth look at the CS (Current steering) DAC architecture, which is the starting point of the time-interleaved architecture. First the limitations of the CS DAC were discussed; both its static and its dynamic limitations. This was followed by an overview of recent literature regarding techniques to improve CS DAC performance.

In chapter 3 the basic architecture which is used in the rest of this thesis was presented: the two-times interleaved DAC. This interleaved DAC consists of two sDACs in parallel of which alternatingly one is connected to the output once stabilized, while the other one is connected to a dummy output while switching to the next output code. The operating principle of this architecture was introduced, including how this suppresses non-linearities of the sDACs. In the rest of chapter 3 the performance limitations of the interleaved DAC are analyzed: mismatch between sDAC amplitude, mismatch in the multiplexer's duty cycle and code-dependent behavior of the multiplexer's transistors. A method was introduced to estimate the sub-picosecond timing error using solely DC comparisons, which can be used to remove the timing-error using calibration. 
This was followed in chapter 4 by a demonstrator implementation in $65 \mathrm{~nm}$ bulk CMOS. This 12-bit interleaved DAC operates at $1.7 \mathrm{GS} / \mathrm{s}$ while achieving $>58 \mathrm{~dB}$ SFDR and $<-62 \mathrm{dBC}$ IM3 over Nyquist. The timing for interleaving is adjustable using a digitally programmable capacitor bank, while a novel way to calibrate the current sources to obtain good matching between the two sDACs was presented. Using this calibration algorithm, the linearity requirements on the ADC used for calibration are virtually absent while the inter-sDAC matching was shown to improve by a factor of 150 . The effectiveness of the interleaved architecture was demonstrated by comparing a single SDAC linearity to the overall interleaved DAC performance: Besides the doubling of the sampling rate by placing two sDACs in parallel, also IM3 performance of Nyquist is boosted by almost $20 \mathrm{~dB}$. While this comes at the cost of increased power consumption and area, since two sDACs and an analog multiplexer need to be used, the overall system is still power and area efficient due to the low demands on the sDACs.

In chapter 5 another implementation was presented, aimed at high-speed while maintaining reasonable linearity and only using limited area and power. The quadswitching DAC was introduced in the interleaved architecture, where quad-switching dramatically reduces demands on power supply and bias nodes, while the interleaved architecture suppresses timing errors inherit to the quad-switching architecture. The effectiveness of the design was shown by a $28 \mathrm{~nm}$ FDSOI implementation running at $11 \mathrm{GS} / \mathrm{s}$ and by a $65 \mathrm{~nm}$ bulk CMOS implementation running at $8.8 \mathrm{GS} / \mathrm{s}$. Both achieved $>50 \mathrm{~dB}$ SFDR and <-50dBc IM3 over Nyquist, while only using approximately $100 \mathrm{~mW}$. The $28 \mathrm{~nm}$ implementation occupied $0.04 \mathrm{~mm}^{2}$ while the $65 \mathrm{~nm}$ implementation required $0.074 \mathrm{~mm}^{2}$ die area.

Combined, in this thesis the feasibility and advantages of an interleaved DAC were introduced, described and demonstrated. While the interleaved architecture also has disadvantages, options were presented to deal with those disadvantages while maintaining the advantages. It was shown that this interleaving DAC system can be used to design high-speed DACs with good linearity and a small foot print and power consumption.

\subsection{Future work}

The work presented in this thesis is shown to be effective for the design of high-speed DACs. This can be further improved upon; in the following sections some options are discussed. 


\subsubsection{Signal swing}

The interleaved architecture presented in this work operates best with a relatively low output swing. With an increasing output swing, the gate-source voltage variation of the multiplexing triode transistors also increases. At high output voltage levels, the gatesource voltage is limited by the maximum voltage that is allowed by the used process in order to avoid (mainly) oxide breakdown. At low output voltage levels, hence at a low gate-source voltage, the on-resistance of the triode mosfets is large, and eventually the mosfet will enter saturation region instead of triode which increases non-linearity.

Ideally the gate-source voltage of these transistors should be relatively constant, even with a large output swing. This would mean the gate voltage preferably has to move with the source voltage, and hence needs to be variable.

\subsubsection{Bootstrap}

One way to implement this is by using a bootstrap circuit that should keep the gatesource voltage constant. However bootstrap circuits typically suffer from two problems. The first problem is the increase in complexity: the capacitive level shifter used in the demonstrator designs in this thesis is simple to implement, and the signal chain is simply a string of inverters followed by a capacitor. This simplicity allows it to operate very fast. Meanwhile a bootstrap circuit would have a lot of switches which would need to operate in different phases.

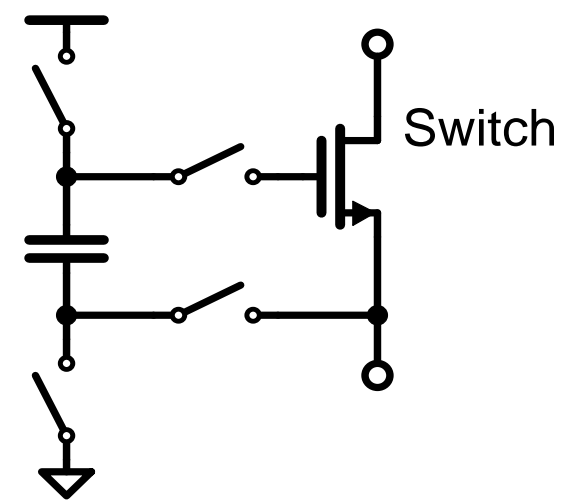

Figure 6-1: Bootstrap circuit

The second problem is the timing of the bootstrap circuit. When bootstrap circuits are employed in track and hold circuits for ADCs the critical edge is the edge which opens the switch, so the edge which discharged the tracking transistor. Making this edge very accurate timing wise is fairly straightforward. However since the DAC output is 
continuous time, also the other edge, which closes the multiplexer switch, needs to be accurate. This means the bootstrap transistor needs to be switched into the circuit at a very accurate, level independent, fashion. In practice this is extremely hard to achieve.

\subsubsection{Multiple level shifter}

An alternative would be using a variable level shifter instead of a fixed one. A possible implementation of this is shown in figure 6-2.

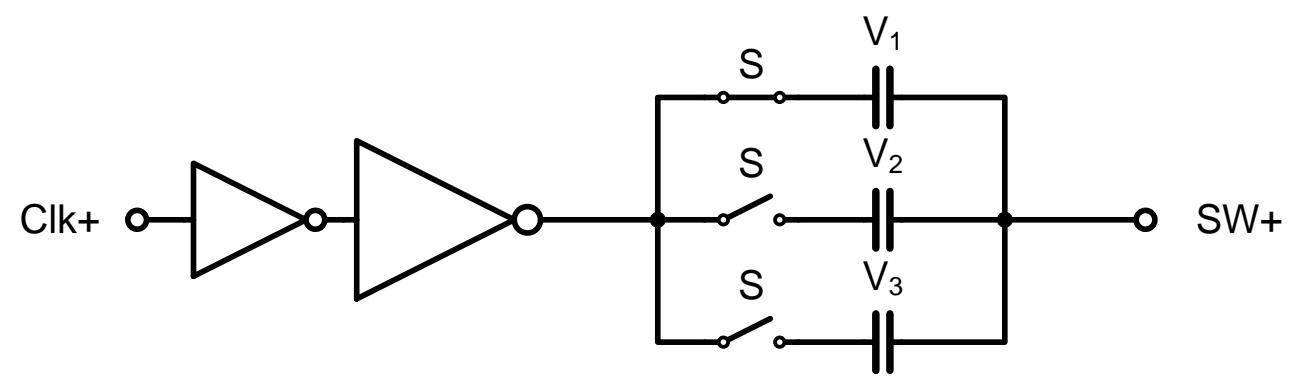

Figure 6-2: Variable level shifter where each capacitor is charged to a different voltage

Instead of a single capacitor that operates as a level shifter, several capacitors can be used, each charged to a different voltage. A multiplexer chooses which of the capacitors is used, which allows for a staircase approximation of a bootstrap output.

The resulting staircase approximation is expected to be sufficiently accurate: the overdrive voltage of the multiplexer transistors does not need to be perfectly constant, the goal is just to make sure they stay deep within triode region. There are also other challenges that need to be solved with this approach: the outputs of the multiplexer inside the level shifter experiences voltage swings outside the power supply. The voltage levels used need to be properly synchronized with the outputs of the sDACs and charge injection spikes that will occur when changing the shifted level are a potential source of linearity degradation.

\subsubsection{Transformer}

A simpler solution to increase the power the DAC can deliver to its output is adding a (wideband) transformer at the output, which can be designed to have a low swing at its input but a high swing at its output. In order to do this a much higher current is required, however while the interleaved architecture is limited in tolerable output 
voltage swing, increasing the output current at constant swing should not pose problems.

An on-chip transformer has normally high losses compared to an off-chip transformer and takes a lot of chip area. Off-chip transformers are a lot more efficient, but off-chip components are undesired in general.

\subsubsection{RFPA}

A similar solution to the transformer option is adding an off-chip RF PA in a commongate/base configuration, which can present the DAC with a low impedance load, decreasing the swing for the multiplexing transistors while still allowing a high output swing. The downside is similar to that of a transformer: it requires an off-chip component. An advantage compared to using a transformer is that less current is required to get a high signal swing, simplifying the circuit design. At the same time it does mean a higher supply voltage is required to deliver the same amount of power as with a transformer. An extra bias current added to the signal current will be required to keep the RF PA in its proper operating region.

\subsubsection{Background timing calibration}

Both implemented designs used foreground calibration to remove the timing error in the analog multiplexer: At start-up the timing error was compensated, after which it was assumed to be temperature/voltage independent. While during measurements this has been sufficient, where the time between measurement and calibration has been several days, a background calibration scheme that continuously corrects the error would be advantageously.

The two implemented duty cycle adjustment methods both allow for continuous modifications of the duty cycle. However since the measurement of the timing error is done using a specialized waveform (square wave), this cannot be done in regular operation.

An alternative is instead of evaluating the duty cycle at the output, to evaluate it at the output of the final inverters in the multiplexer buffer, right before the level shifters. From the inverted square waves the duty cycle error can be estimated with the DC measurement method introduced in section 3.2.

A downside of this method is that errors due to differences in threshold voltage of the multiplexer switches are not taken into account and hence are not compensated for. 
Since introducing differences in threshold voltage is shown in chapter 5 to be effective way to modify the duty cycle, it follows that unwanted differences can also introduce a duty cycle error which are ignored when the measurement is done at the output of the inverters. Whether this will be a major implementation issue will depend on the actual circuit design, its requirements and the matching properties in the used technology.

A foreground calibration method also takes into account the amplitude error of the two SDACs, and compensates this for near-Nyquist frequencies, this is further discussed in Appendix A. When background calibration using the above proposed method is implemented this does not occur, and demands on SDAC matching increase.

So it will depend on the design whether background or foreground calibration is preferred, and more extensive research would be required to verify the concepts. 


\section{Appendix A \\ Co-existing timing and amplitude errors}

In this appendix a derivation is made for the theoretical SFDR of a two-times interleaved DAC which suffers from both amplitude and timing mismatch. This allows for additional insight in how these add together and how they can be used to (partially) cancel out. Besides the amplitude and timing mismatch, no further non-idealities are included for this derivation and an ideal zero-order hold model is used.

Initially the Fourier transform is calculated on the zero-order hold output of the two sDACs separately, where we normalize their waveforms. Both are represented with discrete-time domain impulse waveforms. The amplitude of sDAC-A is $1-\triangle A$, while that of sDAC-B is $1+\triangle A$. They are centered around zero, with a normalized width of 1 . sDAC$A$ has a width of $1-\Delta t$, and SDAC-B a width of $1+\Delta t$. They are described by:

$$
\begin{aligned}
& f_{A}=(1-\Delta A)\left(H\left(t+\frac{1-\Delta t}{2}\right)-H\left(t-\frac{1-\Delta t}{2}\right)\right) \\
& f_{B}=(1+\Delta A)\left(H\left(t+\frac{1+\Delta t}{2}\right)-H\left(t-\frac{1+\Delta t}{2}\right)\right)
\end{aligned}
$$

On these waveforms the Fourier transform is applied, given by:

$$
\begin{aligned}
& F(\omega)=\int_{-\infty}^{\infty} f(t) e^{-j \omega t} d t \\
& \omega=2 \pi f
\end{aligned}
$$


The result is given by:

$$
\begin{aligned}
& F_{A}(f)=(1-\Delta A) \frac{\sin (\pi \mathrm{f}) \cos (\pi \mathrm{f} \Delta \mathrm{t})-\cos (\pi \mathrm{f}) \sin (\pi \mathrm{f} \Delta \mathrm{t})}{\pi f} \\
& F_{B}(f)=(1+\Delta A) \frac{\sin (\pi \mathrm{f}) \cos (\pi \mathrm{f} \Delta \mathrm{t})+\cos (\pi \mathrm{f}) \sin (\pi \mathrm{f} \Delta \mathrm{t})}{\pi f}
\end{aligned}
$$

The wanted signal is the sum of these two transforms, while the mismatch, so the difference, between them is the unwanted half-Nyquist mirror that needs to be suppressed.

So the fundamental signal is:

$$
\begin{aligned}
F_{\text {fund }}(f)=F_{A}(f) & +F_{B}(f) \\
= & 2 \frac{\sin (\pi \mathrm{f}) \cos (\pi \mathrm{f} \Delta \mathrm{t})+\Delta \mathrm{A} \cos (\pi \mathrm{f}) \sin (\pi \mathrm{f} \Delta \mathrm{t})}{\pi f}
\end{aligned}
$$

Since $\Delta \mathrm{t}$ is small $\cos (\pi \mathrm{f} \Delta \mathrm{t})$ approaches one, and can be removed. Compared to the first term, the second term with both a small $\Delta A$ and a small $\sin (\pi f \Delta t)$ is also neglible, which leaves the standard zero-order hold spectrum for the fundamental, only with amplitude 2 due to the used definitions:

$$
F_{\text {fund }}(f)=2 \frac{\sin (\pi f)}{\pi f}
$$

The image frequency is the difference between the two transforms, where the frequency needs to be taken into account: the image starts at Nyquist $(f=1 / 2$ with the used definitions), and with increasing fundamental frequency it decreases towards DC.

$$
\begin{gathered}
F_{\text {image }}(f)=F_{B}\left(\frac{1}{2}-f\right)-F_{A}\left(\frac{1}{2}-f\right) \\
F_{\text {image }}(f)=2 \frac{\cos \left(\pi f_{2}\right) \sin \left(\pi f_{2} \Delta t\right)+\Delta A \sin \left(\pi f_{2}\right) \cos \left(\pi f_{2} \Delta t\right)}{\pi f_{2}} \\
f_{2}=\frac{1}{2}-f
\end{gathered}
$$


Since $\Delta \mathrm{t}$ is very small, the first sin can be replaced by a linear approximation, which holds close to zero. The last cos can be removed completely, it will be very close to one.

$$
F_{\text {image }}(f)=2 \frac{\pi f_{2} \Delta t \cos \left(\pi f_{2}\right)+\Delta A \sin \left(\pi f_{2}\right)}{\pi f_{2}}
$$

This can be rewritten to:

$$
\begin{array}{r}
A \sin (x)+B \cos (x)=\sqrt{A^{2}+B^{2}} \sin \left(x+\operatorname{atan}\left(\frac{B}{A}\right)\right) \\
F_{\text {image }}(f)=\frac{2}{\pi f_{2}} \sqrt{\pi^{2} f_{2}^{2} \Delta t^{2}+\Delta A^{2}} \sin \left(\pi f_{2}+\operatorname{atan}\left(\frac{\pi f_{2} \Delta t}{\Delta A}\right)\right)
\end{array}
$$

For $f=F s / 2$ an amplitude error can be compensated by adding an opposite timing error. This will be the case when the quasi-DC timing calibration is performed.

$$
\begin{gathered}
\Delta A=\epsilon \\
\Delta t=-\epsilon
\end{gathered}
$$

Combining these two equations:

$$
F_{\text {image }}(f)=2 \epsilon \sqrt{1+\frac{1}{\pi^{2} f_{2}^{2}}} \sin \left(\pi f_{2}-\operatorname{atan}\left(\pi f_{2}\right)\right)
$$

From this it follows that the magnitude of the spur is directly proportional to the error, differences in the error will not affect the frequency transfer. So using this information we can plot the SFDR, and know that a different $\epsilon$ will only shift the resulting graph, and not change the shape. Below this is done for a compensated amplitude inequality of $1 \%$, which means $\epsilon=0.005$. 


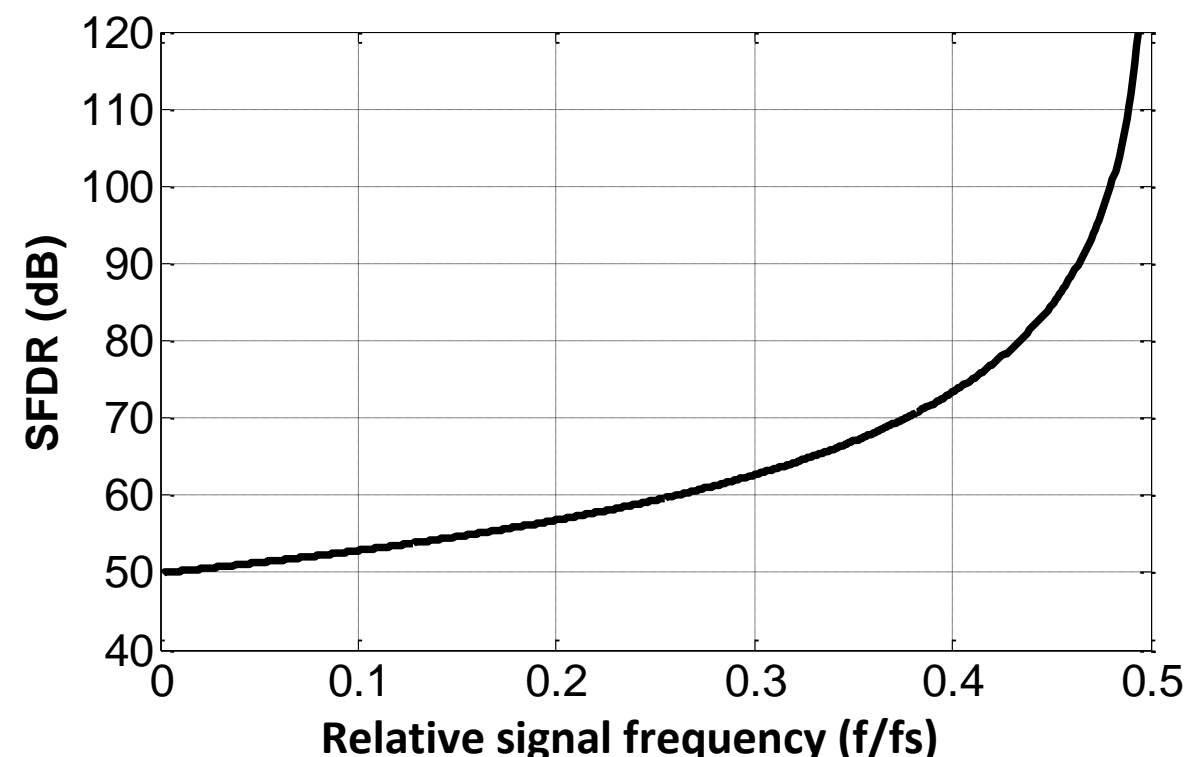

Figure A-1: Ideal SFDR with an amplitude inequality of $1 \%$, compensated by duty cycle calibration

This shows that under ideal circumstances the timing calibration makes sure that the amplitude error is worst at DC, and improves with increasing signal frequency. 


\section{Appendix B \\ On-chip memory architecture}

For testing the DACs a digital (multi-tone) sine wave is required. The $65 \mathrm{~nm} 1.7 \mathrm{GS} / \mathrm{s}$ DAC from chapter 4 employs a synthesized digital sine generator for that purpose. However while that is sufficient for those relative low speeds, the $65 \mathrm{~nm} 9 \mathrm{GS} / \mathrm{s}$ and the $28 \mathrm{~nm}$ FDSOI 11GS/s DACs from chapter 5 have a too high clock frequency to use synthesized logic for that purpose. As alternative solution these chips use an on-chip memory which can be programmed with the required test signals. The architecture of this memory is discussed below.

\section{B.1 Basic architecture}

The designed memory for the DAC in chapter 5 is effectively a ring-buffer which operates at the clock speed of an sDAC. For each sDAC slice (a current source, quadswitches and the switch signal generation and buffers), 63 D-flip-flops are placed in a ring: every clock cycle each bit moves one position further. This architecture is shown in figure b-1. Somewhere in the ring an extra connection is placed which moves the data towards the quad-switching signal generation. This output connection can be placed anywhere.

This architecture has several advantages. First of all the critical path is extremely short: there is no logic between two flip-flops: the data only needs to be able to arrive at the next flip-flop in time without additional delays due to logic gates. Because a ring is made, there are some longer connections to close the circle, but the delays added due to the longer connections are small. Since each clock cycle each bits moves one position, the load on the power supply is virtually code independent. It does depend on the specific digital sine loaded into the memory, but once it is loaded the same amount 
of transitions happen each clock cycle. This reduces possible errors in the analog part of the DAC due to power supply/ground loading, and also due to substrate noise.

The architecture also suffers from some limitations. It is very power hungry: it consist of a lot of flip-flops which are active all the time. However since this is only used to test the actual DAC this power consumption can be ignored as long as it does not result in thermal problems. A larger problem is the clock distribution. Since each flip-flop needs to move the data at the same time, also the clock needs to arrive at the same time for each flip-flop. This means a large central clock buffer needs to provide the clock signals, and these must be distributed to each flip-flop. This sets a limitation to the size of the memory which can be made this way. Another limitation is that a flip-flop is a large circuit to store a single bit: the size of the memory on the chip is also relatively large, which is shown in figure $b-2$.

Because it is a repetitive pattern this can be lay-outed by hand and made suitable for high frequency operation. The downsides means that the size of the implemented memory is limited.

\section{B.2 Memory implementation}

The implemented memory is split in two memory banks: each of the sDACs has its each 63-word memory running at opposite clock phases at half the DACs overall sample rate, resulting in a combined 126 word memory. The output of the memory is buffered by two more sets of flip-flops before the data is transported to the actual SDACs. The memory runs on a separate clock from the sDACs: they are driven by the same source, but have different delays. To prevent any timing issues between SDAC clocking and the memory clocking there is the option to invert the clock that drives the memory; effectively shifting the memory clock phase by half a period.

Having a 63 word memory means it is easy to connect the clocks using a binary tree, while there are fewer sine waves which result in a repetitive pattern than with a 64 word SDAC memory.

The memories are shown in figure b- 2 for the $65 \mathrm{~nm}$ implementation. They are similar in $28 \mathrm{~nm}$, however due to the tiling on that chip they are less well visible. 


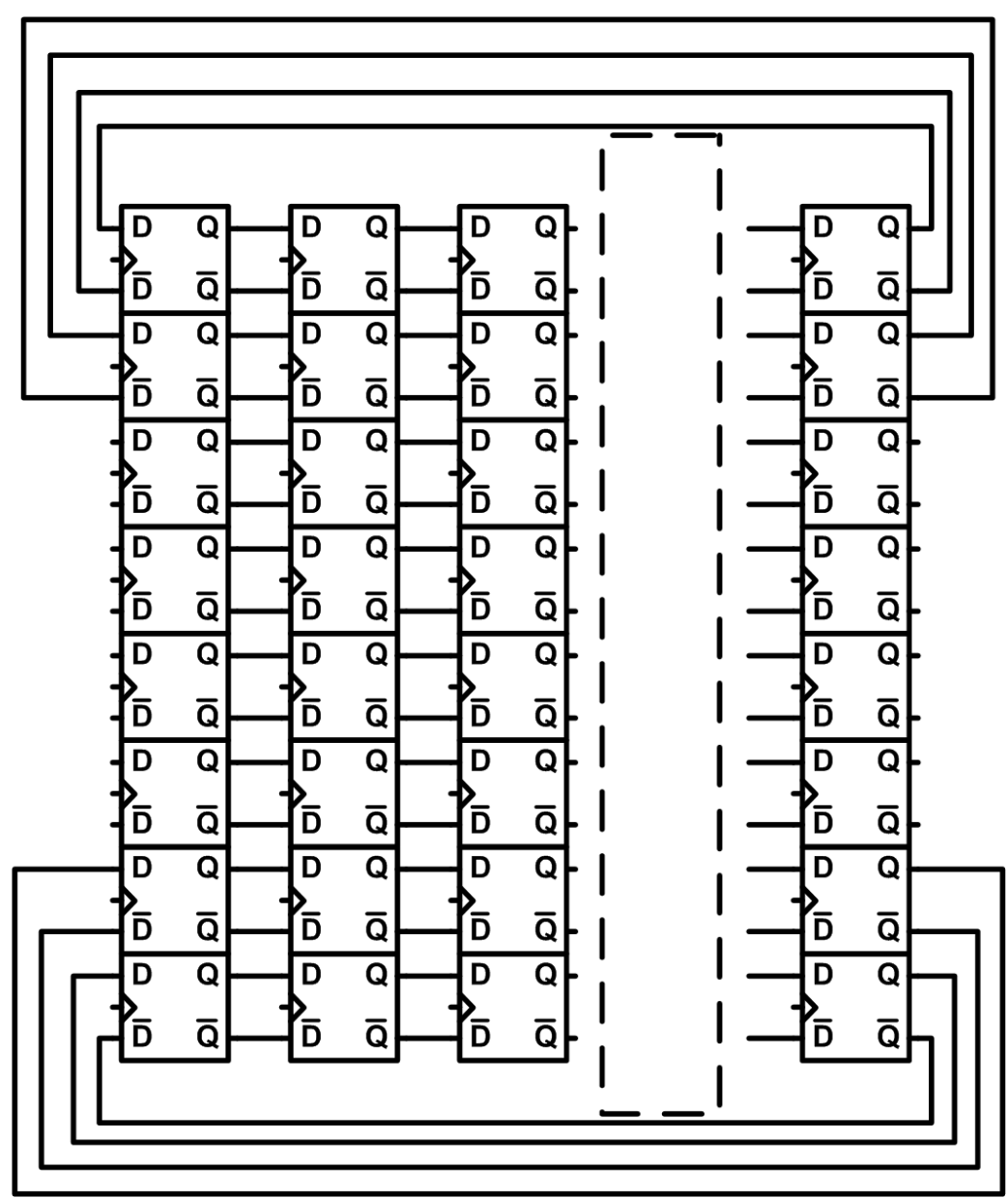

Figure B-1: Memory architecture

\section{B.3 Programming the memory}

Programming the memory is essential to be able to use it. A UTControl bus is implemented on the chip which is used for programming settings, and is also used to program the memory. The programming mode of the memory consist of two parts: in the clock line a multiplexer is placed which allows the user to choose between the regular, full-speed, clock, or a programming clock which can be toggled by the user when there is new data to shift into the memory. Shifting data into the memory is done by a set of multiplexers which can break the memory-ring: the flip-flops belonging to one word can either obtain the new data from the previous memory location, or from an external register. 


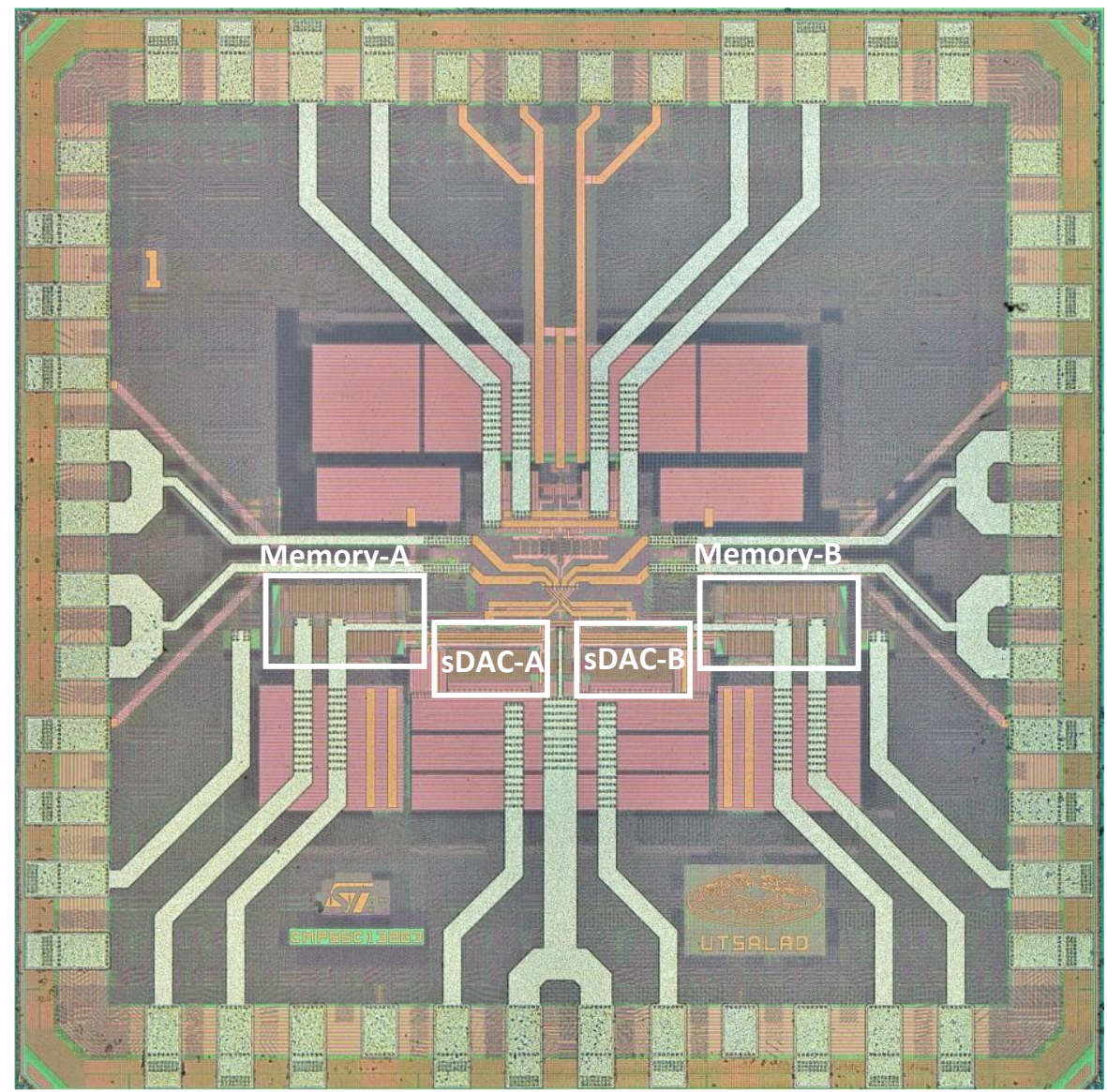

Figure B-2: Die photograph of the 65nm 9-bit DAC with the memories and the sDACs marked

After programming, the memory needs to revert back to regular operation. Since there are two memory blocks these need to be correctly synced: if the memory belonging to one sDAC starts before its turn the samples will not be reproduced in the correct order. In order to prevent this a central block distributes the memory clock which makes sure both blocks start in the correct phase and at the same time.

There is also another problem when it switches back to the regular clock. Figure B-3 shows this issue: if the clock multiplexer switches from programming clock to regular clock, it is very well possible there is a small spike on the multiplexers output, which clocks the memory. This can be interpreted as a clock edge by some flip-flips, while others ignore it, corrupting the memory contents. 


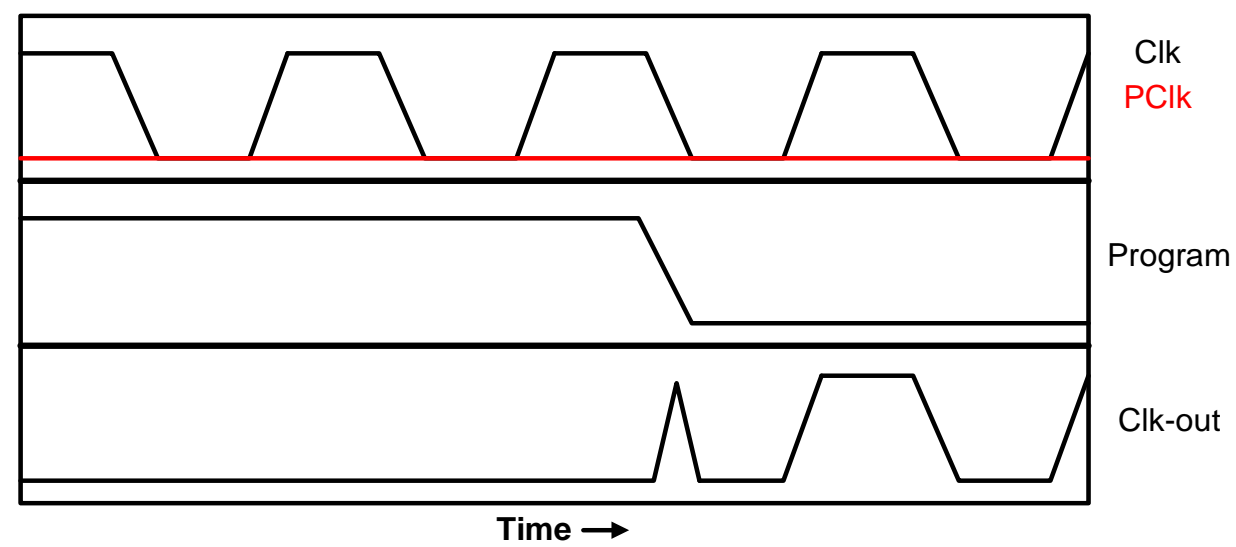

Figure B-3: Switching from programming clock to regular clock

The circuit from Figure B-4 is designed to prevent this. The regular clock and programming clock of both memory blocks are XOR'ed: when they are equal to each other their output is low, otherwise it is high. A NAND gate afterwards combines these two signals; its output is high when for both memory blocks the regular clock and the programming clock is equal. When this happens a rising edge is generated and a flipflop will move the programming mode to the multiplexer settings. This means that only when for both memory blocks the programming clock and the regular clock become equal to each other, the clock settings can be modified, preventing short spikes on the clock outputs. 


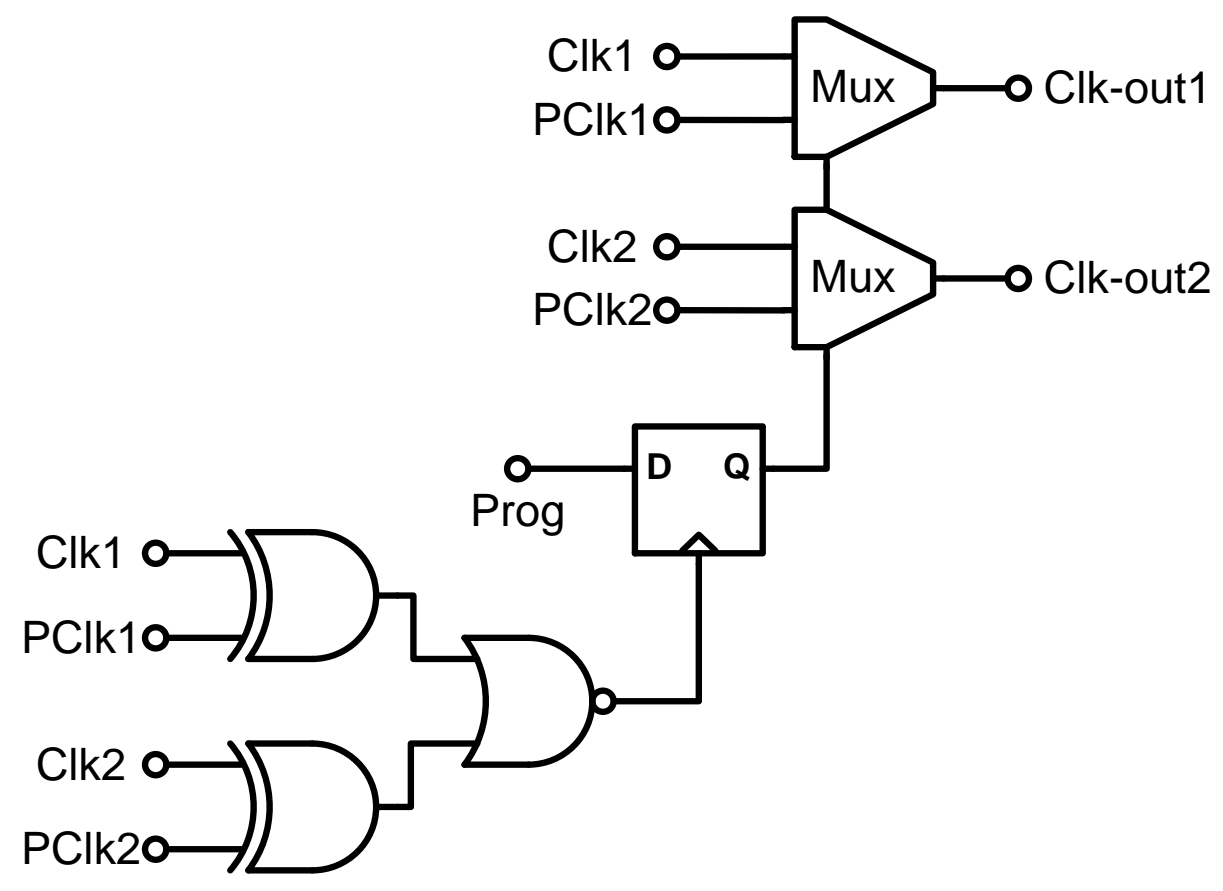

Figure B-4: Clock selection circuit 


\section{Appendix C \\ Layout considerations regarding symmetry}

In this thesis the requirements regarding matching between the two sDACs has been discussed. The importance of matching both the amplitude and the timing of the interleaved sDACs has been shown, where mismatch causes interleaving spurs which can limit the overall SFDR. However symmetry errors in the layout can also result in other errors.

The $65 \mathrm{~nm} 1.7 \mathrm{GS} / \mathrm{s}$ DAC described in chapter 4 was actuallythe second version, compared to the first version the layout was modified, where one of the main reasons was to improve symmetry in the layout of the DAC. The floor plan of the original design is shown in Figure $\mathrm{C}-1$.

\section{C.1 Problem definition}

In the first version the multiplexer buffer was horizontally placed near the bias of one of the sDACs. The SDAC, including its bias, and the multiplexer buffer are connected to different power domains, however the chip uses a shared ground, and this is a short connection to the bias, while the connection to the other bias is a much longer connection. This means that the disturbance on the ground line of the left bias generator is a lot larger than that of the right bias generator. 


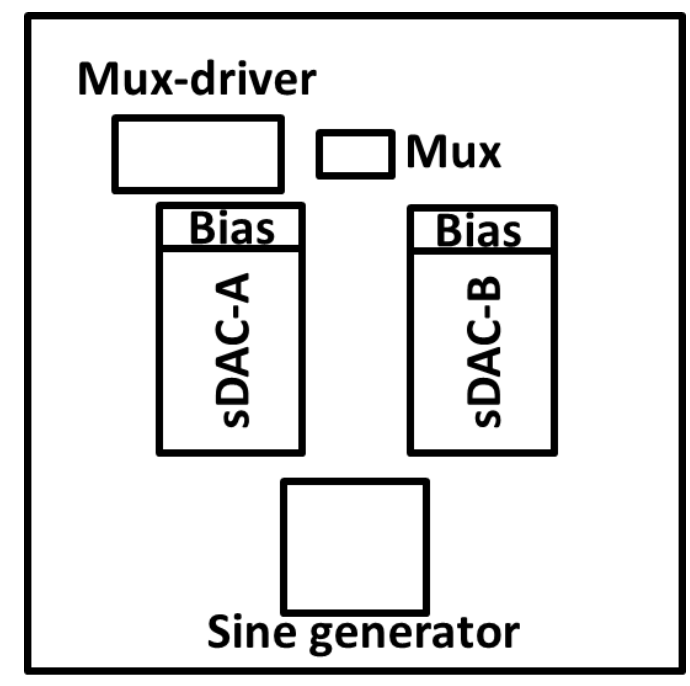

Figure C-1: Floor plan of the initial design

The multiplexer buffer runs at half the overall sampling frequency. However since it is a differential buffer the load on the shared ground line is at the full sample frequency. If this disturbance would have been shared identically across the entire chip there would have been no issues: the mix products of a disturbance at the full sample rate and the fundamental signal end up at the same frequencies as regular image products. However in this case they couple a lot stronger to one half of the interleaved DAC than to the other half, which generates extra image frequencies which the other half does not generate, resulting in imperfect cancellation of these images, and hence additional interleaving spurs.

These spurs are independent of the fundamental frequency as expected: since they add an AC modulation on the output current the amplitude of the extra generated image frequencies do not depend on the fundamental frequency. There is a relation with the sampling frequency, since at higher frequencies the ground is less well defined.

Because both the spur due to this layout effect and the mismatch between sDAC amplitudes is independent of the fundamental output frequency and they occur at the same location, it might be possible to include an extra amplitude error which could cancel the layout induced spur. 


\section{C.2 Analysis}

Figure C-2 shows the three situations to consider. First there is the amplitude mismatch, this we can compensate for, but we can also introduce it to counteract the two other situations: a sine wave disturbance at the sampling frequency, with and without a 90 degrees phase shift. Other phase shifts would be a combination of these two situations.
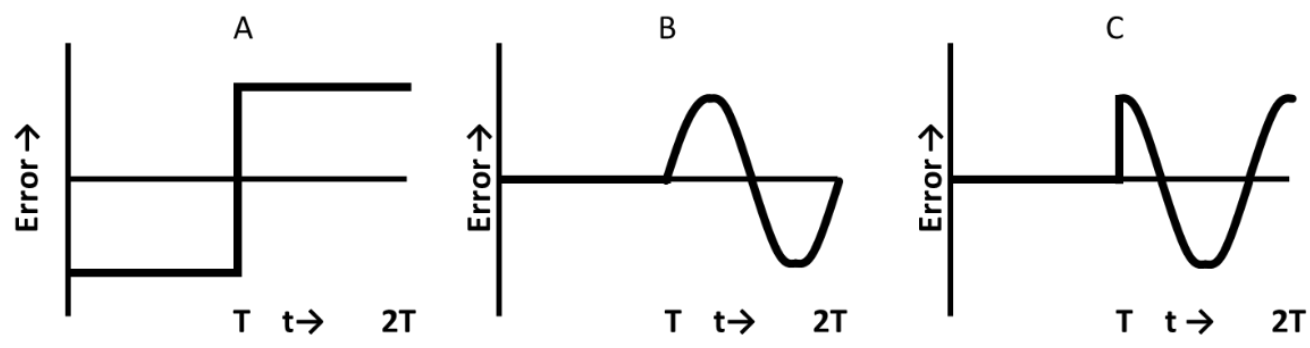

Figure C-2: Errors due to mismatch between sDACs: a) amplitude error, b) sine disturbance at one half, c) sine disturbance at one half with 90 degrees phase shift

To analyze this further the FFT of these signals has been taken. The most important spectral component is the component at Fs/2, while this still falls outside Nyquist, this component will multiply the fundamental frequency to create extra spurs inside Nyquist. In all three situations this spur is present, which hints at the possibility of cancelling the spur by introducing an amplitude error into the sDACs. However this is not always an option: while all three situations do include spurs at $\mathrm{Fs} / 2$, the phases of these spurs are not identical. The phase of the $\mathrm{Fs} / 2$ tone for cases $A$ and $C$ is 90 degrees. So situation $C$ appears to be cancellable. However the phase for case $B$ is zero degrees: It is orthogonal to the tone generated by $\mathrm{A}$, and adding more amplitude error can only make it worse and cannot compensate for it.

In practice the same was noticed during measurements: it was not possible to cancel the extra interleaving spur by introducing more amplitude error. So most likely situation $B$ dominates this layout induced error.

\section{C.3 Solution}

In the new floor plan, shown in Figure C-3, the multiplexer buffer was rotated 90 degrees, which makes the entire layout a lot more symmetrical. Since this includes the pinout, also the PCB could be a lot more symmetrical. In the new layout the ground of the multiplexer buffer is connected at the top side to the ground of the padring. First of all the increased distance, with several ground connections on the padring to the 
external ground, reduces the direct coupling to the ground of the bias block. Second because the connection is symmetrical the coupling is equal to both bias blocks, which causes the generated in-band spurs of the two SDACs to cancel each other, further suppressing them.

In this way the second, described in chapter 4, version could suppress the interleaving spurs far enough to make sure they did not limit performance.

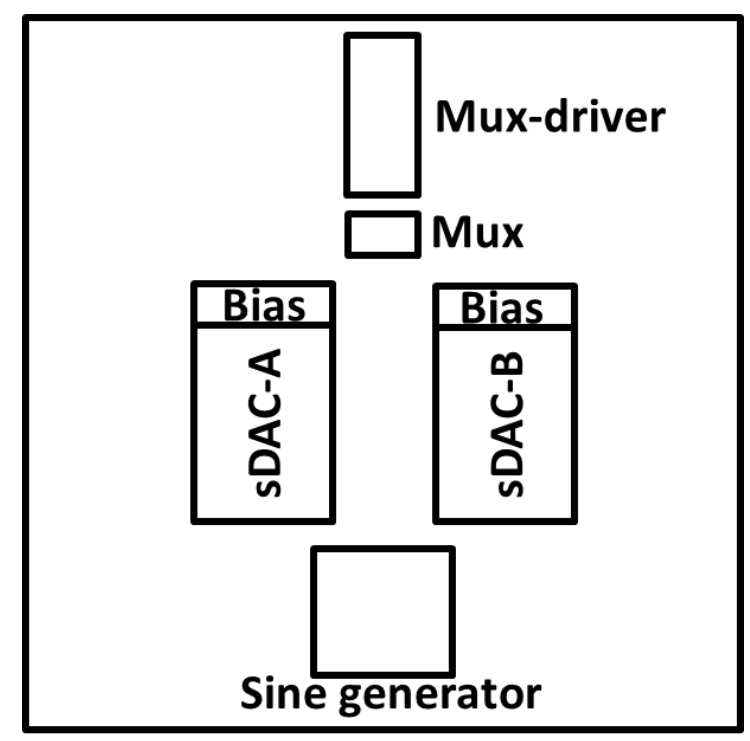

Figure C-3: Floor plan of the improved design 


\section{Appendix D \\ FDSOI for Current steering DACs}

Compared to bulk CMOS, FDSOI (Fully Depleted Silicon-On-Insulator) offers several advantages. Some of these advantages have little relevance to an average current steering DAC, however others do provide benefits in the design of CS DACs.

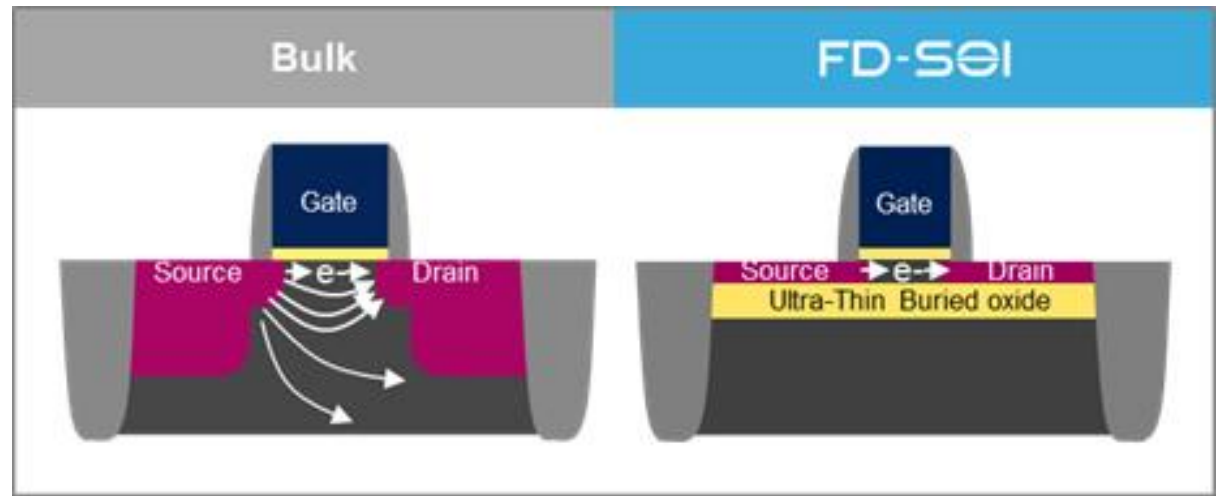

Figure D-1: Bulk CMOS compared to FDSOI, figure from [68]

Compared to regular bulk CMOS FDSOI has two major differences. An ultra-thin BOx (Buried Oxide) is added compared to the bulk device, which makes it a SOI (silicon-oninsulator) device. This isolates both the source and drain areas and the channel from the underlying substrate/well. In addition the channel is undoped, resulting in the fullydepleted channel of the mosfet [69]. Compared to bulk CMOS this gives some new options and modifications in transistor behavior, the ones relevant to CS DACS are discussed here. 


\section{D.1 Drain to bulk junction}

Regular bulk CMOS transistors have parasitic junctions from source and drain to the bulk node. In general the capacitance of these junctions lower the obtainable speed of circuits, and specifically for DACs the large output capacitance of current sources can be a problem. While the ultra-thin BOx of the used FDSOI technology does not completely eliminate this capacitance, it is greatly reduced [69].

Depending on the required specifications this might also remove the need for cascode transistors to isolate the current source capacitance.

\section{D.2 Output resistance}

Besides a low output capacitance, current sources also require a high output resistance to approach their ideal behavior. This is especially important if a design without cascode transistors would be implemented. FDSOI transistors have compared to bulk devices better intrinsic gain, especially for long devices [70]. When designing current sources this results in a higher output resistance.

\section{D.3 Matching}

The channel of an FDSOI transistor does not contain dopants, which also means it is not affected by random fluctuations of the dopant concentration. Compared to bulk CMOS this improves device matching significantly [71]. As discussed in previous chapters of this thesis, both current and timing matching are essential for good performance. Depending on the available process, bulk CMOS can achieve improved matching of current sources by employing native mosfets, however these have larger minimum dimensions than regular mosfets, making them unsuitable for use in the dynamic sections of a DAC. 


\section{Dankwoord}

En dan komen we aan bij het allerlaatste stukje van dit proefschrift. Al zal het voor de meeste lezers waarschijnlijk het allereerste stuk zijn, het dankwoord. Dit proefschrift is uiteraard niet zonder hulp en ondersteuning van andere tot stand gekomen.

Om bij het begin te beginnen: Bedankt AJ! Als mijn dagelijks begeleider is Anne-Johan Annema mijn eerste hulp bij ICD problemen geweest. En bij twijfels was er uiteraard altijd de kalibratiehamer beschikbaar.

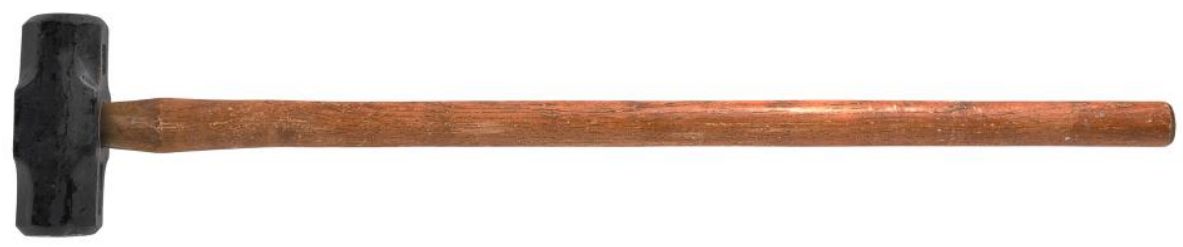

Niet de kalibratiehamer uit dit verhaal.

Vervolgens komen we automatisch bij mijn promotor, Bram Nauta. Bram heeft zowel nuttige ideeën geleverd voor het werk zelf als ook praktische hulp. Als we het over praktische hulp hebben dan hebben we het over de uiteindelijk bazin van ICD: Gerdien. Zonder wiens help bij allerlei praktische problemen het AIO leven een stuk lastiger was geweest. Natuurlijk mag ik ook Henk en Gerard niet vergeten voor hun hulp en kunde. En niet te vergeten de andere STARS gerelateerden: Remco, Eric en Frank.

Special thanks go to Ankur Bal, Pratap Narayan Singh, Anupam Jain, Neha Bhargava, Ranbir Singh and Nitin Chhabra. Their custom design of an integrated sine generator greatly helped with evaluating the performance of the DAC presented in chapter 4 . More help came from STM in the form of Andreia Cathelin and Stéphane Le Tual who presented useful feedback during the various design reviews and of course for the 
silicon which was donated by STMicroelectronics. Also the assistance from CMP is much appreciated.

De dagelijkse dosis aan ondersteuning, humor en tijdverdrijf is geleverd door mijn kamergenoten: Bram, Mark, Michiel, Jasper, David, Harish, Johan, Inês en Yuan-Ching. En speciale dank gaat naar Hugo en Dirk-Jan, die niet alleen in het kamergenoten rijtje thuishoren, maar daarnaast ook als paranymphen hebben geholpen bij de laatste loodjes.

Naast onze kamer was er nog een kamer die belangrijk is geweest tijdens mijn AIO tijd, de koffiekamer. Geen wereldprobleem was te groot om ver genoeg versimpeld te worden zodat er oplossingen bedacht konden worden (niet altijd even praktische oplossingen overigens, maar dat is ook niet de bedoeling in de academische wereld). Allemaal werd dat mogelijk gemaakt door de mede bewoners van de koffiekamer: Ray, Tom, TomTom, Sander, Jurriaan, Remke, Dirk en nog vele andere permanente en tijdelijke koffiekamerbewoners.

Als laatste, maar zeker niet als minste, wil ik ook mijn familie, en specifiek mijn ouders, bedanken voor hun steun tijdens zowel mijn carrière als PhD'er. 


\section{Bibliography}

[1] 2010. [Online]. Available: http://starsproject.nl/.

[2] I. Kuon and J. Rose, "Measuring the Gap Between FPGAs and ASICs," ComputerAided Design of Integrated Circuits and Systems, IEEE Transactions on, vol. 26, no. 2, pp. 203-215, Feb 2007.

[3] E. Lee and P. Gulak, "A CMOS field-programmable analog array," Solid-State Circuits, IEEE Journal of, vol. 26, no. 12, pp. 1860-1867, Dec 1991.

[4] "AN231E04 Datasheet Rev 1.2," 2014. [Online]. Available: http://www.anadigm.com/_doc/DS231000-U001.pdf.

[5] S. Balasubramanian and W. Khalil, "Direct digital-to-RF digital-to-analogue converter using image replica and nonlinearity cancelling architecture," Electronics Letters, vol. 46, no. 14, pp. 1030-1032, July 2010.

[6] N. Friedman, The Naval Institute Guide to World Naval Weapon Systems, Naval Institute Press, 2006, p. 265.

[7] E. Vittoz, "Low-power design: ways to approach the limits," in Solid-State Circuits Conference, 1994. Digest of Technical Papers. 41st ISSCC., 1994 IEEE International, 1994.

[8] Y.-H. Kim and H. Yu, "Automatic tuning circuit for Gm-C filters," in Electronics, Circuits and Systems, 2005. ICECS 2005. 12th IEEE International Conference on, 2005.

[9] S. Smith, The Scientist and Engineer's Guide to Digital Signal Processing, California Technical Publishing, 1997.

[10] M. Skolnik, Radar Handbook, 3rd ed., McGraw-Hill, 1990.

[11] S. Cripps, RF power amplifiers for wireless communications, 2nd ed., Artech House, Incorporated, 2006.

[12] J. Velner, E. Klumperink, B. Nauta and F. van Vliet, "Multi-band linear chirp generation based on a type-III PLL," in Microwave Symposium Digest (IMS), 2013 IEEE MTT-S International, 2013. 
[13] F. Maloberti, Data Converters, Springer, 2007, pp. 254-255.

[14] M. Oude Alink, A. Kokkeler, E. Klumperink, K. Rovers, G. Smit and B. Nauta, "Spurious-Free Dynamic Range of a Uniform Quantizer," Circuits and Systems II: Express Briefs, IEEE Transactions on, vol. 56, no. 6, pp. 434-438, June 2009.

[15] G. Engel, S. Kuo and S. Rose, "A 14b 3/6GHz current-steering RF DAC in 0.18 um CMOS with $66 \mathrm{~dB}$ ACLR at 2.9GHz," in Solid-State Circuits Conference Digest of Technical Papers (ISSCC), 2012 IEEE International, 2012.

[16] R. Walden, "Analog-to-digital converter survey and analysis," Selected Areas in Communications, IEEE Journal on, vol. 17, no. 4, pp. 539-550, April 1999.

[17] R. Schreier and G. Temes, Understanding Delta-Sigma Data Converters, New York: Wiley, 2005.

[18] A. Ali, A. Morgan, C. Dillon, G. Patterson, S. Puckett, P. Bhoraskar, H. Dinc, M. Hensley, R. Stop, S. Bardsley, D. Lattimore, J. Bray, C. Speir and R. Sneed, "A 16bit 250-MS/s IF Sampling Pipelined ADC With Background Calibration," Solid-State Circuits, IEEE Journal of, vol. 45, no. 12, pp. 2602-2612, Dec 2010.

[19] D. Baranauskas and D. Zelenin, "A 0.36W 6b up to 20GS/s DAC for UWB Wave Formation," in Solid-State Circuits Conference, 2006. ISSCC 2006. Digest of Technical Papers. IEEE International, 2006.

[20] "Low-power 14-bit current steering DAC, for ADSL2+/CO applications in 0.13 um CMOS," in Solid-State Circuits Conference, 2004. ESSCIRC 2004. Proceeding of the 30th European, 2004.

[21] R.-L. Chen and S.-J. Chang, "A 6-bit Current-Steering DAC With Compound Current Cells for Both Communication and Rail-to-Rail Voltage-Source Applications," Circuits and Systems II: Express Briefs, IEEE Transactions on, vol. 59, no. 11, pp. 746-750, Nov 2012.

[22] W.-T. Lin and T.-H. Kuo, "A 12b 1.6GS/s 40mW DAC in 40nm CMOS with >70dB SFDR over entire Nyquist bandwidth," in Solid-State Circuits Conference Digest of Technical Papers (ISSCC), 2013 IEEE International, 2013.

[23] J. Wikner, Studies on CMOS Digital-to-Analog Converters, Linköping University Electronic Press, 2000.

[24] K. Doris, A. van Roermund and D. Leenaerts, Wide-Bandwidth High Dynamic Range D/A Converters, Springer, 2006.

[25] J. McCreary and P. Gray, "All-MOS Charge Redistribution Analog-to-Digital Conversion Techniques-Part I," Solid-State Circuits, IEEE Journal of, vol. 10, no. 6, pp. 371-379, Dec 1975.

[26] R. Suarez, P. Gray and D. Hodges, "All-MOS charge-redistribution analog-to-digital conversion techniques. II," Solid-State Circuits, IEEE Journal of, vol. 10, no. 6, pp. 379-385, Dec 1975. 
[27] L. Wang, Y. Fukatsu and K. Watanabe, "A CMOS R-2R ladder digital-to-analog converter and its characterization," in Instrumentation and Measurement Technology Conference, 2001. IMTC 2001. Proceedings of the 18th IEEE, 2001.

[28] K. Poulton, B. Jewett and J. Liu, "A 7.2-GSa/s, 14-bit or 12-GSa/s, 12-bit DAC in a 165-GHz fT BiCMOS process," in VLSI Circuits (VLSIC), 2011 Symposium on, 2011.

[29] M. Pelgrom, "A 10-b 50-MHz CMOS D/A converter with 750hm buffer," SolidState Circuits, IEEE Journal of, vol. 25, no. 6, pp. 1347-1352, Dec 1990.

[30] A. Bugeja, B.-S. Song, P. Rakers and S. Gillig, "A 14-b, 100-MS/s CMOS DAC designed for spectral performance," Solid-State Circuits, IEEE Journal of, vol. 34, no. 12, pp. 1719-1732, December 1999.

[31] S. Luschas and H.-S. Lee, "Output impedance requirements for DACs," in Circuits and Systems, 2003. ISCAS '03. Proceedings of the 2003 International Symposium on, 2003.

[32] A. D. A. W. M.J.M Pelgrom, "Matching properties of MOS transistors," Solid-State Circuits, IEEE Journal of, vol. 24, no. 5, pp. 1433-1439, October 1989.

[33] M. Kosunen and K. Halonen, "Sampling jitter and power supply interference in current-steering D/A converters," in Circuit Theory and Design, 2005. Proceedings of the 2005 European Conference on, 2005.

[34] C.-H. Lin, F. van der Goes, J. a. M. J. Westra, Y. Lin, E. Arslan, E. Ayranci, X. Liu and K. Bult, "A 12 bit 2.9 GS/s DAC With IM3 60 dBc Beyond 1 GHz in 65 nm CMOS," Solid-State Circuits, IEEE Journal of, vol. 44, no. 12, pp. 3285-3293, Dec 2009.

[35] B. C. T. Y. K. N.-Y. W. X. C. T.-H. C. K. R. H.-F. C. R. G. J. C. J. Xiao, "A 13-Bit 9GS/s RF DAC-based broadband transmitter in 28nm CMOS," pp. C262-C263, June 2013.

[36] A. Bugeja and S. B.-S., "A self-trimming 14-b 100-MS/s CMOS DAC," Solid-State Circuits, IEEE Journal of, vol. 35, no. 12, pp. 1841-1852, December 2000.

[37] W.-H. Tseng, C.-W. Fan and J.-T. Wu, "A 12b 1.25GS/s DAC in 90nm CMOS with > 70dB SFDR up to 500MHz," in Solid-State Circuits Conference Digest of Technical Papers (ISSCC), 2011 IEEE International, 2011.

[38] W.-H. Tseng, J.-T. Wu and Y.-C. Chu, "A CMOS 8-Bit 1.6-GS/s DAC With Digital Random Return-to-Zero," Circuits and Systems II: Express Briefs, IEEE Transactions on, vol. 58, no. 1, pp. 1-5, Jan 2011.

[39] W.-T. Lin, H.-Y. Huang and T.-H. Kuo, "A 12-bit 40 nm DAC Achieving SFDR $>70 \mathrm{~dB}$ at $1.6 \mathrm{GS} / \mathrm{s}$ and IMD $<-61 \mathrm{~dB}$ at $2.8 \mathrm{GS} / \mathrm{s}$ With DEMDRZ Technique," Solid-State Circuits, IEEE Journal of, vol. 49, no. 3, pp. 708-717, March 2014.

[40] K. Nguyen, R. Adams and K. Sweetland, "A 113 dB SNR oversampling sigma-delta DAC for CD/DVD application," Consumer Electronics, IEEE Transactions on, vol. 44, no. 3, pp. 1019-1023, Aug 1998. 
[41] S. Park, G. Kim, S.-C. Park and W. Kim, "A digital-to-analog converter based on differential-quad switching," Solid-State Circuits, IEEE Journal of, vol. 37, no. 10, pp. 1335-1338, Oct. 2002.

[42] B. Schafferer and R. Adams, "A 3V CMOS $400 \mathrm{~mW} 14 \mathrm{~b} 1.4 \mathrm{GS} / \mathrm{s}$ DAC for multicarrier applications," in Solid-State Circuits Conference, 2004. Digest of Technical Papers. ISSCC. 2004 IEEE International, 2004.

[43] S. Wan, Z. Chen, Z. Yu, S. Huang and H. Ji, "An embedded 14-bit 800MS/s DAC for direct digital frequency synthesizer in 0.18-um CMOS," in Applied Superconductivity and Electromagnetic Devices, 2009. ASEMD 2009. International Conference on, 2009.

[44] B. Brandt, D. McMahill, M. Wu, P. Kalthoff, A. Kuckreja and G. Ostrem, "A 14b 4.6GS/s RF DAC in 0.18 um CMOS for cable head-end systems," in Solid-State Circuits Conference Digest of Technical Papers (ISSCC), 2014 IEEE International, 2014.

[45] S. Balasubramanian, G. Creech, J. Wilson, S. M. Yoder, J. J. McCue, M. Verhelst and W. Khalil, "Systematic Analysis of Interleaved Digital-to-Analog Converters," Circuits and Systems II: Express Briefs, IEEE Transactions on, vol. 58, no. 12, pp. 882-886, Dec 2011.

[46] X. Li, Q. Wei, Z. Xu, J. Liu, H. Wang and H. Yang, "A 14 Bit 500 MS/s CMOS DAC Using Complementary Switched Current Sources and Time-Relaxed Interleaving DRRZ," Circuits and Systems I: Regular Papers, IEEE Transactions on, vol. 61, no. 8, pp. 2337-2347, Aug 2014.

[47] G. Radulov, P. Quinn, P. Harpe, H. Hegt and A. van Roermund, "Parallel currentsteering D/A Converters for Flexibility and Sma," in Circuits and Systems, 2007. ISCAS 2007. IEEE International Symposium on, 2007.

[48] D. Feng, S. Sin, E. Bonizzoni and F. Maloberti, "Time interleaved current steering DAC for ultra-high conversion rate," in Microelectronics and Electronics (PRIME), 2014 10th Conference on Ph.D. Research in, 2014.

[49] F. Y. H.-F. Y. N. L. J. X. J.-Y. R. L. Cheng, "Nyquist-rate time-interleaved currentsteering DAC with dynamic channel matching," in Circuits and Systems (ISCAS), 2011 IEEE International Symposium on, 2011.

[50] D. Aksin, G. Ozbek and F. Maloberti, "Multi-rate segmented time-interleaved current steering DAC with unity-elements sharing," in Circuits and Systems (ISCAS), Proceedings of 2010 IEEE International Symposium on, 2010.

[51] L. Cheng, F. Ye, H.-F. Yang, N. Li, J. Xu and J.-Y. Ren, "Nyquist-rate time-interleaved current-steering DAC with dynamic channel matching," in Circuits and Systems (ISCAS), 2011 IEEE International Symposium on, 2011.

[52] K. Doris, J. Briaire, D. Leenaerts, M. Vertreg and A. van Roermund, "A 12b $500 \mathrm{MS} / \mathrm{s}$ DAC with $>70 \mathrm{~dB}$ SFDR up to $120 \mathrm{MHz}$ in 0.18 um CMOS," in Solid-State 
Circuits Conference, 2005. Digest of Technical Papers. ISSCC. 2005 IEEE International, 2005.

[53] X. Wu, P. Palmers and M. Steyaert, "A $130 \mathrm{~nm}$ CMOS 6-bit Full Nyquist $3 \mathrm{GS} / \mathrm{s}$ DAC," Solid-State Circuits, IEEE Journal of, vol. 43, no. 11, pp. 2396-2403, Nov 2008.

[54] J. Deveugele and M. Steyaert, "A 10-bit 250-MS/s binary-weighted currentsteering DAC," Solid-State Circuits, IEEE Journal of, vol. 41, no. 2, pp. 320-329, Feb 2006.

[55] S. Tual, P. Singh, A. Bal and C. Garnier, "A 3GS/s, 9b, 1.2V single supply, pure binary DAC with >50dB SFDR up to $1.5 \mathrm{GHz}$ in 65nm CMOS," in VLSI Circuits (VLSIC), 2011 Symposium on, 2011.

[56] S. Su, T. Tsai, P. Sharma and M.-W. Chen, "A 12-bit hybrid DAC with 8GS/s unrolled pipeline delta-sigma modulator achieving $>75 \mathrm{~dB}$ SFDR over $500 \mathrm{MHz}$ in $65 \mathrm{~nm}$ CMOS," in VLSI Circuits Digest of Technical Papers, 2014 Symposium on, 2014.

[57] W.-T. Lin and T.-H. Kuo, "A Compact Dynamic-Performance-Improved CurrentSteering DAC With Random Rotation-Based Binary-Weighted Selection," SolidState Circuits, IEEE Journal of, vol. 47, no. 2, pp. 444-453, Feb 2012.

[58] T. Chen and G. Gielen, "A 14-bit 200-MHz Current-Steering DAC With SwitchingSequence Post-Adjustment Calibration," Solid-State Circuits, IEEE Journal of, vol. 42, no. 11, pp. 2386 -2394, November 2007.

[59] H. Van de Vel, J. Briaire, C. Bastiaansen, P. van Beek, G. Geelen, H. Gunnink, Y. Jin, M. Kaba, K. Luo, E. Paulus, B. Pham, W. Relyveld and P. Zijlstra, "A 240mW 16b $3.2 \mathrm{GS} / \mathrm{s}$ DAC in $65 \mathrm{~nm}$ CMOS with $<-80 \mathrm{dBc}$ IM3 up to $600 \mathrm{MHz}$," in Solid-State Circuits Conference Digest of Technical Papers (ISSCC), 2014 IEEE International, 2014.

[60] S.-S. Chen, N.-S. Kim and J. Rabaey, "A 10b 600MS/s multi-mode CMOS DAC for multiple Nyquist zone operation," in VLSI Circuits (VLSIC), 2011 Symposium on, 2011.

[61] E. Olieman, A.-J. Annema and B. Nauta, "An Interleaved Full Nyquist High-Speed DAC Technique," Solid-State Circuits, IEEE Journal of, vol. 3, no. 50, pp. 704-713, 2015.

[62] J. Deveugele, P. Palmers and M. Steyaert, "Parallel-path digital-to-analog converters for Nyquist signal generation," Solid-State Circuits, IEEE Journal of, vol. 39, no. 7, pp. 1073-1082, July 2004.

[63] M. Clara, W. Klatzer, D. Gruber, A. Marak, B. Seger and W. Pribyl, "A 1.5V 13bit $130-300 \mathrm{MS} / \mathrm{s}$ self-calibrated DAC with active output stage and $50 \mathrm{MHz}$ signal bandwidth in 0.13um CMOS," in Solid-State Circuits Conference, 2008. ESSCIRC 2008. 34th European, 2008. 
[64] D. Spicer and A. Rodger, "Antiglitch Digital to Analog Converter System". United states Patent 3,877,023, 8 April 1975.

[65] E. Olieman, A.-J. Annema, B. Nauta, A. Bal and P. Singh, "A 12b 1.7GS/s two-times interleaved DAC with <-62 dBC IM3 across Nyquist using a single $1.2 \mathrm{~V}$ supply," in Solid-State Circuits Conference (A-SSCC), 2013 IEEE Asian, Singapore, 2013.

[66] E. Olieman, A.-J. Annema and B. Nauta, "A $110 \mathrm{~mW}, 0.04 \mathrm{~mm} 2,11 \mathrm{GS} / \mathrm{s}$ 9-bit interleaved DAC in 28nm FDSOI with $>50 \mathrm{~dB}$ SFDR across Nyquist," in VLSI Circuits (VLSIC), 2011 Symposium on, 2014.

[67] Y. Greshishchev, D. Pollex, S.-C. Wang, M. Besson, P. Flemeke, S. Szilagyi, J. Aguirre, C. Falt, N. Ben-Hamida, R. Gibbins and P. Schvan, "A 56GS/S 6b DAC in $65 \mathrm{~nm}$ CMOS with 256x6b memory," in Solid-State Circuits Conference Digest of Technical Papers (ISSCC), 2011 IEEE International, 2011.

[68] STMicroelectronics, "FD-SOI," [Online]. Available: http://www.st.com/web/en/about_st/learn_fd-soi.html.

[69] P. Magarshack, P. Flatresse and G. Cesana, "UTBB FD-SOI: A process/design symbiosis for breakthrough energy-efficiency," in Design, Automation Test in Europe Conference Exhibition (DATE), 2013, 2013.

[70] P. Magarshack, FD-SOl: From Successful Collaborative R\&D to Successful Silicon Results, 2013.

[71] X. Federspiel, D. Angot, M. Rafik, F. Cacho, A. Bajolet, N. Planes, D. Roy, M. Haond and F. Arnaud, "28nm node bulk vs FDSOI reliability comparison," in Reliability Physics Symposium (IRPS), 2012 IEEE International, 2012. 


\section{List of Publications}

1. E. Olieman, A.-J. Annema, B. Nauta, A. Bal and P. Singh, "A 12b 1.7GS/s twotimes interleaved DAC with <-62dBc IM3 across Nyquist using a single $1.2 \mathrm{~V}$ supply," Solid-State Circuits Conference (A-SSCC), 2013 IEEE Asian, pp.81-84, 11-13 Nov. 2013

2. E. Olieman, A.-J. Annema and B. Nauta, "A 110mW, 0.04mm2, 11GS/s 9-bit interleaved DAC in 28nm FDSOI with >50dB SFDR across Nyquist", VLSI Circuits (VLSIC), 2011 Symposium on, pp. 206-207, 11-13 June 2014

3. E. Olieman, A.-J. Annema and B. Nauta, "An Interleaved Full Nyquist HighSpeed DAC Technique," Solid-State Circuits, IEEE Journal of, vol. 3, no. 50, pp. 704-713, 2015. 



\section{List of Abbreviations}

\begin{tabular}{|c|c|}
\hline ADC & Analog-to-Digital Converter \\
\hline ASIC & Application-Specific Integrated Circuit \\
\hline $\mathrm{BOx}$ & Buried Oxide \\
\hline BW & BandWidth \\
\hline CE & Constant Envelope \\
\hline CML & Current Mode Logic \\
\hline CMOS & Complementary Metal-Oxide Semiconductor \\
\hline CPU & Central Processing Unit \\
\hline CS & Current steering \\
\hline DAC & Digital-to-Analog Converter \\
\hline DC & Direct Current \\
\hline DMRZ & Dynamic-element-Matching and Return-to-Zero \\
\hline DNL & Differential Non-Linearity \\
\hline DRRZ & Digital Random Return-to-Zero \\
\hline DSP & Digital Signal Processor \\
\hline FDSOI & Fully Depleted Silicon-On-Insulator \\
\hline FoM & Figure-of-Merit \\
\hline FPAA & Field-Programmable Analog Array \\
\hline FPGA & Field-Programmable Gate Array \\
\hline IF & Intermediate Frequency \\
\hline IM3 & Third-Order Inter-Modulation \\
\hline INL & Integral Non-Linearity \\
\hline NMOS & N-type Metal-Oxide Semiconductor \\
\hline NRZ & Non-Return-to-Zero \\
\hline PMOS & P-type Metal-Oxide Semiconductor \\
\hline RZ & Return-to-Zero \\
\hline sDAC & sub-DAC \\
\hline SFDR & Spurious-Free Dynamic Range \\
\hline SNR & Signal-to-Noise Ratio \\
\hline $\mathrm{GS} / \mathrm{s}$ & Giga-samples per second \\
\hline
\end{tabular}

